Aus dem Fachbereich Medizin

der Johann Wolfgang Goethe-Universität

Frankfurt am Main

betreut am

Zentrum der Gesundheitswissenschaften

Institut für Allgemeinmedizin

Direktor: Prof. Dr. Ferdinand M. Gerlach, MPH

\title{
Landpartie 2.0 - Konzeptionelle Entwicklung und Implementierung eines longitudinalen Schwerpunktprogramms zur Förderung der
} Allgemeinmedizin im ländlichen Raum.

\author{
Dissertation \\ zur Erlangung des Doktorgrades der theoretischen Medizin \\ des Fachbereichs Medizin \\ der Johann Wolfgang Goethe-Universität \\ Frankfurt am Main \\ vorgelegt von \\ Linda Seeger (geb. Barthen) \\ M. Sc. Public Health \\ aus Staßfurt
}

Frankfurt am Main, 2020 
Dekan:

Referent:

Korreferent:

Tag der mündlichen Prüfung:
Prof. Dr. Stefan Zeuzem

Prof. Dr. Ferdinand M. Gerlach, MPH

Prof. Dr. Dr. h.c. David Groneberg

18. März 2021 


\section{Inhaltsverzeichnis}

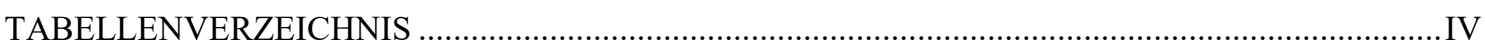

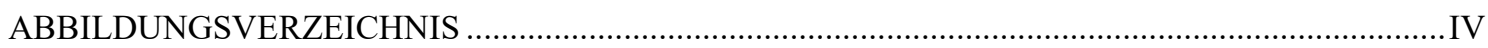

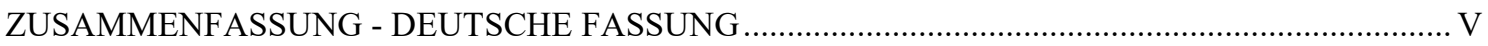

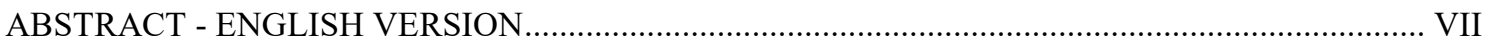

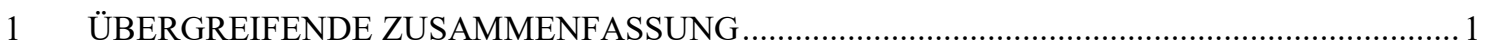

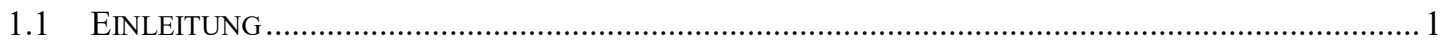

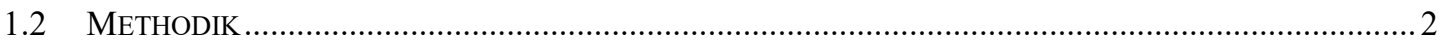

1.3 DARSTELlUng UND ZENTRALE ERgEBNISSE DER PUBLIKATIONEN ...................................................

1.3.1 Publikation 1: Vorarbeiten und Befragung aller medizinischen Fakultäten in

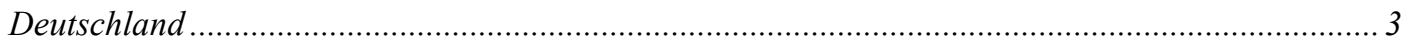

1.3.2 Publikation 2: Befragung der Medizinstudierenden der Goethe-Universität....................... 5

1.3.3 Publikation 3: Konzeptionelle Entwicklung und Implementierung der Landpartie 2.0........ 6

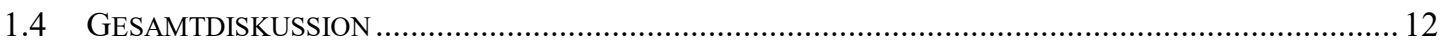

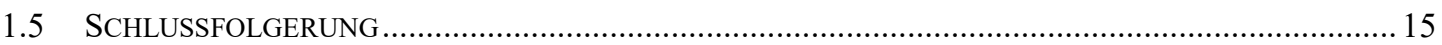

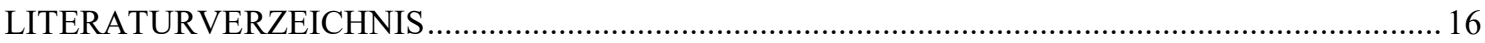

ÜBERSICHT DER VERÖFFENTLICHTEN PUBLIKATIONEN .............................................................IX

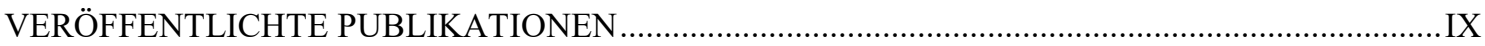

DARSTELLUNG DES EIGENEN ANTEILS AN DEN EINZELNEN PUBLIKATIONEN............XLVIII

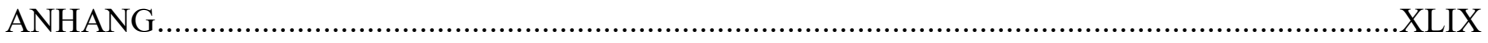

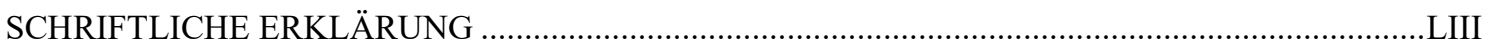




\section{Tabellenverzeichnis}

TABELLE 1: Sozio-demografische Angaben zum Bewerbungszeitpunkt

\section{Abbildungsverzeichnis}

ABBILDUNG 1: Curricularer Ablauf der "Landpartie 2.0" im klinischen

Studienabschnitt

ABBILDUNG 2: Evaluationsergebnisse der Praxisphasen, der Seminarreihe und des Tagesausflugs bis Sommersemester 2019 


\section{Zusammenfassung - Deutsche Fassung}

Die Sicherstellung der hausärztlichen Versorgung ist vor allem im ländlichen Raum mit regional unterschiedlich starker Ausprägung zunehmend gefährdet. Ein wesentlicher Grund liegt in der stetig sinkenden Zahl an hausärztlich tätigen Ärzten/innen. Ursächlich hierfür sind einerseits die hohen „Bruttoabgänge“ von Hausärzten/innen, zumeist aufgrund altersbedingten Ausscheidens, und einem andererseits eklatanten Nachwuchsproblem.

Um dieser problematischen Entwicklung entgegenzuwirken, kommt der Gewinnung hausärztlichen Nachwuchses eine Schlüsselrolle zu. In Flächenländern wie Australien, Kanada oder den USA, die ähnlichen Herausforderungen schon seit längerer Zeit gegenüberstehen, existieren seit den 1970er Jahren universitäre Schwerpunktprogramme, die die Allgemeinmedizin bereits in der medizinischen Ausbildung fördern. Breit angelegte Evaluationsstudien zeigen dabei, dass die Teilnahme an longitudinalen Längsschnittcurricula einen positiven Effekt auf die Wahrscheinlichkeit hat, nach Abschluss des Studiums eine Weiterbildung im Fach Allgemeinmedizin aufzunehmen und sich darüber hinaus hausärztlich (im ländlichen Raum) niederzulassen.

Unter der Annahme, dass eine allgemeinmedizinische Schwerpunktsetzung im Studium das Interesse am selbigen Fach erhöht und darüber hinaus eine hausärztliche Karriereplanung positiv beeinflusst, soll die hier vorliegende Promotionsarbeit folgende Forschungsfrage beantworten: Wie kann ein longitudinales, fachbereichsweites Lehrangebot konzeptionell gestaltet werden, welches es Medizinstudierenden ermöglicht, Allgemeinmedizin im ländlichen Raum kennenzulernen?

Zur Beantwortung der Fragestellung wurde ein triangulierender Forschungsansatz gewählt, der aus mehreren Arbeitsschritten besteht: 1. Erarbeitung einer Übersichtarbeit bestehend aus einer Literaturrecherche und der Kontaktaufnahme zu hiesigen Experten, 2. schriftliche und telefonische Befragung aller medizinischen Fakultäten Deutschlands 3. webbasierte Befragung von Medizinstudierenden der Goethe Universität, Frankfurt, 4. konzeptionelle Entwicklung und Implementierung eines universitären Schwerpunktprogramms zur Förderung der Allgemeinmedizin in ländlichen Regionen auf Basis der in Schritt eins bis drei gewonnen Ergebnisse.

Mittels der verschiedenen methodischen Entwicklungsschritte konnte im Zeitraum von 2015 bis 2016 das longitudinale Schwerpunktprogramm „Landpartie 2.0“ konzeptionell 
entwickelt und ab dem Wintersemester 2016/2017 in das Medizinstudium der GoetheUniversität, Frankfurt am Main, implementiert werden.

Das entwickelte Lehrangebote richtet sich pro Jahr in der Regel an bis zu 15 Studierende ab dem klinischen Studienabschnitt. Im Kern beinhaltet das mehrsemestrige Angebot wiederkehrende Praxisphasen in ausgewählten und geschulten Hausarztpraxen in ländlichen Regionen. Begleitet werden die Praktika von vor- und nachbereitenden Seminaren an der Universität, dem Kurs Allgemeinmedizin in einer ländlichen Hausarztpraxis und einem jährlichen Tagesausflug zu innovativen Gesundheitsmodellen. Seit Einführung des Programms konnten 62 Studierende in die „Landpartie 2.0“ aufgenommen werden.

Erste Evaluationsergebnisse belegen eine sehr hohe Zufriedenheit mit den einzelnen Programmbestandteilen unter den Teilnehmenden. Langfristig sollen darüber hinaus in einer Verbleibstudie Effekte auf die Motivation für eine hausärztliche Tätigkeit (auf dem Land) sowie Karriereverläufe abgebildet werden.

Insgesamt erwies sich das gewählte methodische Vorgehen als zielführend. Mittels der einzelnen Entwicklungsschritte konnte ein abgestimmtes, umfassendes und den wissenschaftlichen Erkenntnissen berücksichtigendes Längsschnittcurriculum am Fachbereich Medizin der Goethe Universität, Frankfurt am Main, erfolgreich konzeptioniert und implementiert werden. 


\section{Abstract - English Version}

It is becoming increasingly difficult to safeguard the provision of primary health care, particularly in rural areas, whereby there is considerable regional variation. The main reason for this is a steady decline in the number of general practitioners caused by agerelated retirement, and enormous problems recruiting young family doctors.

In order to counteract this problematic development, it is important to attract young medical practitioners. In countries such as Australia, Canada and the U.S., which have long faced similar challenges, universities have offered priority programs to promote family medicine since the 1970s. Broad-based evaluation studies have shown that participation in longitudinal curricula has a positive effect on the likelihood that medical graduates will choose to undergo specialist training in family medicine and set up a family practice (in a rural area).

On the assumption that focusing on family medicine during medical studies increases interest in the subject and has a positive influence on the likelihood of pursuing a career in family medicine, this doctoral thesis aims to answer the following research question: How should a longitudinal curriculum to familiarize medical students with family medicine in rural areas be conceptually designed?

The research method chosen to answer the research question was triangulation, whereby it consisted of several work steps: 1. Development of an overview based on a literature search and contact with local experts, 2. Written and telephone surveys of all medical faculties in Germany 3. Web-based surveys of medical students at Goethe University, Frankfurt am Main, 4. Conceptual development and implementation of a university-based priority program to promote family medicine in rural areas based on the results of steps one to three.

Based on the various methodological development steps, a concept for the longitudinal program "Landpartie 2.0" ("Rural Outing 2.0") was developed in 2015 and 2016 and included in medical studies at Goethe University, Frankfurt am Main, from the winter semester of $2016 / 2017$.

The developed curriculum is usually designed for up to 15 students in the clinical stage of their studies. The core of the multi-semester program consists of regular internships at selected, trained family practices in rural regions. The internships are accompanied by 
preparatory and follow-up seminars at the university, a course in family medicine undertaken at a rural family practice, and an annual day trip to become familiarized with innovative health models. Since the program was launched, 62 students have participated in "Landpartie 2.0".

Initial evaluation results have shown that participants' satisfaction with the individual components of the program is very high. The long-term impact of the program on their interest in working as a family practitioner (in a rural area), as well as other career paths they have chosen will be part of a further study.

Overall, the chosen methodological approach proved to be effective. Stage-by-stage, a coordinated, comprehensive, long-term science-based curriculum was successfully developed and implemented at the Medical Faculty of Goethe University, Frankfurt am Main. 


\section{1 Übergreifende Zusammenfassung}

\subsection{Einleitung}

Eine qualitativ hochwertige hausärztliche Versorgung kann in zunehmend mehr Gebieten Deutschlands nicht mehr sichergestellt werden. Insbesondere ländlich geprägte Regionen sind von einer akuten Unterversorgung im Sinne der Bedarfsplanung bedroht. ${ }^{1}$ Dafür ursächlich ist neben einem steigenden medizinischen Versorgungsbedarf (z.B. Zunahme von älteren, chronisch kranken und multimorbiden Patienten/innen), einer veränderten Balance zwischen Arbeit und Freizeit bzw. Familie (z. B. mehr Teilzeitbeschäftigungen) auch ein anhaltender Nachwuchsmangel an jungen Allgemeinmedizinern/innen. ${ }^{2}$ Von den insgesamt 13.336 Facharztanerkennungen im Jahr 2018 entfielen lediglich 11,6 \% (1.567) auf Fachärzte/innen für Allgemeinmedizin. ${ }^{3}$ Im Jahr 1993 lag deren Anteil noch bei 18,8 \%. ${ }^{4}$ Erschwert wird die Situation dadurch, dass sich die Mehrheit der jungen, angehenden Allgemeinmediziner/innen eine Tätigkeit in urbanisierten und infrastrukturell attraktiven Regionen wünscht. ${ }^{5}$

Ein wesentlicher Handlungsschwerpunkt, um diesen Nachwuchsproblemen zu begegnen, besteht in der Förderung der Allgemeinmedizin, welche schon im Studium begonnen werden sollte. ${ }^{6}$

Denn Studien zeigen, dass der frühzeitige und intensivere Kontakt mit der Allgemeinmedizin das Interesse am Fach nachhaltig stärken ${ }^{7}$ und Studierende in ihrem weiteren Karriereweg im Sinne einer hausärztlichen Tätigkeit positiv prägen kann. ${ }^{8}$ Die Entwicklung und Einführung von umfassenden, curricular verankerten Lehrangeboten, die zum Ziel haben, das Interesse der Studierenden an einer hausärztlichen Tätigkeit (im ländlichen Raum) bereits im Studium zu fördern, stellt dabei eine konkrete Maßnahme dar.

Solche universitären Lehrangebote existieren bereits seit einigen Jahrzehnten in großen, ländlich geprägten Flächenländern, wie den USA, Kanada oder Australien. Die Angebotsvielfalt reicht dabei von einzelnen Landarztprogrammen, sogenannten „rural health programs ${ }^{\text {“9-11 }}$, bis hin zu ganzen Universitäten, die sich einzig auf die Aus- und

Weiterbildung von „Landärzten/innen“ (,rural health doctors“) spezialisiert haben. ${ }^{12,13}$ Langzeitevaluationen belegen dabei, dass Absolventen/innen von Landarztprogrammen sich nicht nur häufiger im ländlichen Raum niederlassen ${ }^{14,15}$, sondern auch noch 
Jahrzehnte später häufiger dort tätig sind, als Studienabgänger/innen, die zuvor eine reguläre Aus- und Weiterbildung durchlaufen haben. ${ }^{16}$

Der Förderung allgemeinmedizinischen Nachwuchses wird seit wenigen Jahren auch an deutschen Universitäten mehr Aufmerksamkeit gewidmet. ${ }^{8,17-19}$ Am Fachbereich Medizin der Goethe-Universität Frankfurt am Main können z.B. Studierende seit 2012 ihr „Blockpraktikum Allgemeinmedizin“ in dem eher ländlich geprägten Landkreis Fulda in Hessen absolvieren. Eine begleitende Evaluationsstudie zeigte, dass die Teilnahme an diesem Blockpraktikum die Motivation der Studierenden, später hausärztlich zu arbeiten, erhöht. $^{20}$

Trotz des positiven Ergebnisses, handelt es sich bei der Landpartie Fulda lediglich um ein punktuelles Angebot (zwei Wochen Praktikum im Landkreis Fulda). Weltweite Studienergebnisse zeigen jedoch, dass insbesondere mehrsemestrige, breit angelegte und praxisorientierte Schwerpunktprogramme einen nachweislichen Effekt auf den weiteren Berufsweg von Medizinstudierenden haben. ${ }^{21,22}$ Darüber hinaus werden auch seitens von Fach- und Studienvertretern/innen Forderungen lauter, die Allgemeinmedizin stärker im Studium zu verankern, beispielsweise in Form von freiwilligen Lehrangeboten. ${ }^{23,24}$

Unter der Prämisse, dass praktische Erfahrungen in der Allgemeinmedizin während des Studiums, das Interesse am selbigen Fach nachhaltig positiv beeinflussen und zu einer hausärztlich geprägten Karriereplanung beitragen können, soll deshalb ein über mehrere Semester (longitudinal) bestehendes „Landarztprogramm“ am Fachbereich Medizin der Goethe-Universität, Frankfurt am Main etabliert werden. Im Fokus dieser Zielsetzung steht die Forschungsfrage: Wie kann ein longitudinales, fachbereichsweites Lehrangebot konzeptionell gestaltet werden, welches es Medizinstudierenden ermöglicht, Allgemeinmedizin im ländlichen Raum kennenzulernen?

\subsection{Methodik}

Zur Beantwortung der Fragestellung wird eine Art triangulierender Forschungsansatz gewählt. Hierbei wird der Forschungsgegenstand (die Fragestellung) von mindestens zwei verschiedenen Punkten bzw. methodischen Zugängen betrachtet. Ziel ist dabei nicht die Validität des Ergebnisses zu steigern, sondern vielmehr die Erlangung eines tieferen und reichhaltigeren Verständnisses zur Beantwortung der zugrundeliegenden Fragestellung. In der vorliegenden Arbeit wird dabei auf die von Norman K. Denzin 1970 
definierte „Methodentriangulation“ bzw. davon die „Triangulation zwischen mehreren Methoden“(,,between method“) zurückgegriffen. ${ }^{25}$

Die gewählte Triangulation besteht in der vorliegenden Arbeit aus mehreren Arbeitsschritten:

1. Erarbeitung einer Übersichtarbeit bestehend aus einer Literaturrecherche und der Kontaktaufnahme zu nationalen Experten

2. Schriftliche bzw. telefonische Befragung aller medizinischen Fakultäten Deutschlands in Bezug auf bestehende und geplante universitäre Schwerpunktprogramme zur Gewinnung hausärztlichen Nachwuchses in ländlichen Regionen

3. Webbasierte Befragung von Medizinstudierenden der Goethe-Universität Frankfurt

4. Die konzeptionelle Entwicklung und Implementierung eines universitären Schwerpunktprogramms zur Förderung der Allgemeinmedizin in ländlichen Regionen auf Basis der in Schritt eins bis drei gewonnen Ergebnisse.

Aus dem gewählten methodischen Vorgehen resultierend, umfasst die vorliegende Dissertationsschrift drei Manuskripte, die im Folgenden in ihren jeweiligen Methoden und Ergebnissen vorgestellt werden.

\subsection{Darstellung und zentrale Ergebnisse der Publikationen}

\subsubsection{Publikation 1: Vorarbeiten und Befragung aller medizinischen Fakultäten in Deutschland}

\section{Hintergrund und Fragestellung}

Wie eingangs beschrieben, stellt die Sicherstellung der hausärztlichen Versorgung auch in anderen Ländern eine besondere Herausforderung dar ${ }^{26}$, sodass folglich eine Vielzahl an verschiedenen „Landarztprogrammen“ entstanden ist. ${ }^{9-13}$

Da sich die Zugangsbedingungen zum Medizinstudium sowie die Curricula und Prüfungsformate in den genannten Ländern jedoch teils erheblich vom deutschen Medizinstudium unterscheiden, war eine Adaption trotz positiver Verbleibstudien nicht uneingeschränkt möglich. Aus diesem Grund wurde im weiteren Verlauf das Augenmerk auf die Entwicklungen und den Ist-Stand in Deutschland gelegt. 
Es folgte die Erarbeitung einer Übersichtarbeit bestehend aus einer Literaturrecherche und der Kontaktaufnahme zu hiesigen Experten, die sich bereits zu dem Thema der Förderung der Allgemeinmedizin in ländlichen Regionen während des Studiums verdient gemacht hatten. ${ }^{27,28}$ Obwohl schnell deutlich wurde, dass sich die Lehrangebote in Deutschland in ihrer Intensität nicht mit denen im Ausland vergleichen ließen, zeigte sich ebenfalls, dass das Thema an zunehmend mehr Universitäten Beachtung findet. ${ }^{8,17-20}$ Eine systematische Übersicht, wie viele und welche universitären Schwerpunktangebote in Deutschland bereits existieren, fehlte jedoch. Folglich geht die erste Publikation der Frage nach, welche Angebote zur Förderung der Allgemeinmedizin im ländlichen Raum während des Studiums an deutschen medizinischen Fakultäten bereits existieren oder zum Erhebungszeitpunkt in Planung waren. ${ }^{29}$

\section{Methodik}

Von Juli bis November 2015 wurden alle 37 universitären allgemeinmedizinischen Lehreinrichtungen in Deutschland mittels leitfadengestützten Telefongesprächen und/oder E-Mail befragt. Die Antworten wurden protokolliert und anhand der vorgefertigten und für die Gespräche genutzten Systematik übertragen und strukturiert.

\section{Ergebnisse}

Von den 37 angeschriebenen Einrichtungen nahmen 31 an der Befragung teil (Rücklaufquote $=83,8 \%$ ). An 12 Fakultäten wurden dabei 13 relevante Angebote identifiziert, die sich auf die Förderung der Allgemeinmedizin im Studium fokussieren. Drei davon sind longitudinal bzw. auf eine Laufzeit von mindestens zwei Semestern angelegt. Eine Gemeinsamkeit vieler Angebote ist, dass die Zahl der Plätze pro Jahrgang im Schnitt auf ca. 20 begrenzt ist und die Angebote erst in den vergangenen Jahren entstanden sind. Auffallend war hingegen, dass keines der identifizierten Schwerpunktprogramme explizit damit wirbt, die Allgemeinmedizin im ländlichen Raum zu fördern, so wie es das Ziel des eigenen Forschungsvorhabens war.

\section{Schlussfolgerung/Zwischenfazit}

Auf Basis der gewonnenen Ergebnisse aus der Befragung und den Vorarbeiten (Literaturrecherche sowie Befragung von Experten) konnten erste Ansätze zur Ausgestaltung des geplanten Schwerpunktprogramms entwickelt werden. So wurde beispielsweise entschieden, dass das zu entwickelnde Angebot im Kern wahrscheinlich 
aus Praxisphasen in 1:1-Betreuung und einer begleitenden Seminarreihe an der Universität bestehen wird. Diese Ansätze wurden in der sich anschließenden Befragung der Medizinstudierenden berücksichtigt. Darüber hinaus bieten die Ergebnisse für andere Medizinfakultäten in Deutschland eine Übersicht, welche Programme unter welchen Rahmenbedingungen bereits existieren, sodass diese als Blaupause dienen können.

\subsubsection{Publikation 2: Befragung der Medizinstudierenden der Goethe-Universität}

\section{Hintergrund und Fragestellung}

In die Entwicklung neuer Lehrangebote sind Studierende eher selten involviert. Studien zeigen jedoch, dass die Beteiligung der Zielgruppe am Gestaltungsprozess eine wesentliche Voraussetzung für den Erfolg und die Akzeptanz einer Curriculumsentwicklung oder -änderung ist. ${ }^{30}$ Die Studierenden als Experten/innen in eigener Sache wissen um die Defizite und Potenziale ihres bisherigen Studienplans und sind zudem hoch motiviert, am Veränderungsprozess beteiligt zu werden. ${ }^{31}$ Folglich war es ein wesentliches Anliegen, die Studierenden bei der Erarbeitung eines neuen „Landarztprogrammes“ mit einzubeziehen. Daraus ergibt sich, dass im Fokus der zweiten Publikation die Frage steht, welche Wünsche und Anforderungen die Studierenden selbst an ein Schwerpunktprogramm stellen, das die Allgemeinmedizin im ländlichen Raum longitudinal fördert. ${ }^{32}$

\section{Methodik}

Zur Beantwortung der Fragestellung erfolgte im Sommer 2015 eine webbasierte Befragung im Querschnittsdesign. Der selbstkonzipierte Fragebogen enthielt 19 Items zu den Themenbereichen „Sozio-demographische Angaben“, „Berufswunsch und Studium“ sowie „Angaben $\mathrm{zu}$ dem $\mathrm{zu}$ entwickelnden Schwerpunktprogramm“. Das Messinstrument wurde auf Basis der Ergebnisse aus einer bundesweiten Befragung von Medizinstudierenden erstellt ${ }^{33}$ und in einem interdisziplinären Team abgestimmt. Vor seinem Einsatz wurde der Fragebogen in einem Pretest von Studierenden geprüft. Für die Befragung selbst kam das Umfrage-Tool Survey Monkey zum Einsatz. Die anschließende Auswertung der Daten erfolgte mittels der Statistiksoftware SPSS.

\section{Ergebnisse}

An der Befragung nahmen insgesamt 617 Medizinstudierende der Goethe-Universität Frankfurt ab dem 4. Semester teil (Rücklaufquote $=28,7 \%$ ). Im Ergebnis zeigte sich, 
dass das Interesse an einem potentiellen Angebot groß ist (45,4 \% würden an dem in der Befragung skizzierten Angebot teilnehmen). Dabei erwarten die angehenden Mediziner/innen in erster Linie einen starken Praxisbezug - also vorrangig das Erleben und die aktive Mitarbeit in Hausarztpraxen aber auch die Einführung regelmäßiger praktischer Übungen in möglichen Begleitseminaren. Zudem gehören aus Sicht der Studierenden zu einem solchen Angebot auch das Kennenlernen administrativer sowie wirtschaftlicher Hintergründe zum Führen einer Praxis. Mit Blick auf die beruflichen Perspektiven zeigt sich, dass das grundsätzliche Interesse an einer allgemeinmedizinischen Tätigkeit während des Studienverlaufs zunimmt, wobei jedoch städtische Regionen als bevorzugter Arbeitsort gelten.

\section{Schlussfolgerung/Zwischenfazit}

Die Befragung hat dazu beigetragen, das geplante Schwerpunktprogramm bzgl. Inhalt und Organisation zu konkretisieren. Erste Ideen wurden weiterentwickelt, neue Ansätze generiert und Hinweise berücksichtigt, die zuvor nicht bedacht wurden. So wurde beispielsweise entschieden, dass das Angebot ab dem klinischen Studienabschnitt startet und nicht das gesamte Studium umfasst. Ferner wurden Themen zur Praxisführung und zum Praxismanagement Bestandteile der Seminarreihe. Im Kern sollte das Programm zudem einen kontinuierlichen Praxisbezug aufweisen, der sich auch in den Seminaren in Form von praktischen Übungen zeigt. Es ist zudem zu erwarten, dass die Einbeziehung der Zielgruppe zu einer höheren Akzeptanz des Programms führt.

\subsubsection{Publikation 3: Konzeptionelle Entwicklung und Implementierung der Landpartie 2.0}

In der dritten und abschließenden Publikation im Rahmen der vorliegenden Dissertation, erfolgte die Darstellung des konzeptionell entwickelten Schwerpunktprogramms der „Landpartie 2.0“. Das longitudinale Lehrangebot zur Förderung der Allgemeinmedizin in ländlichen Regionen wurde auf Basis der beschriebenen Arbeitsschritte aus den Publikationen eins und zwei von April 2015 bis September 2016 entwickelt. ${ }^{34}$

\section{Projektbeschreibung und Methodik}

Das fakultative Lehrangebot richtet sich an Studierende des 1. klinischen Semesters. Diese können sich mit einer schriftlichen Bewerbung, die selbstentwickelte, vorgegebene Fragen enthält, auf das Angebot bewerben. Die Auswahl der Teilnehmenden erfolgt dabei auf Grundlage von Prädiktoren, die in einem positiven Zusammenhang mit einer späteren 
Karriereentscheidung der Studierenden in der Allgemeinmedizin ${ }^{35}$ und/oder einer Tätigkeit im ländlichen Raum stehen. ${ }^{36}$ Insgesamt können pro Jahrgang in der Regel bis zu 15 interessierte Studierende in das Programm aufgenommen werden.

Um den zeitlichen Mehraufwand für Studierende möglichst gering zu halten, wurden bereits bestehende curriculare Pflichtlehrveranstaltungen aufgegriffen und organisatorisch sowie zum Teil inhaltlich neu strukturiert. Im Ergebnis absolvieren die Studierenden somit durch ihre Teilnahme am Programm den obligatorischen „Kurs Allgemeinmedizin“, das gesamte „klinische Wahlfach“ sowie das „Blockpraktikum Allgemeinmedizin“ - aber in einem neuen, strukturierten Design (vgl. Abb. 1).

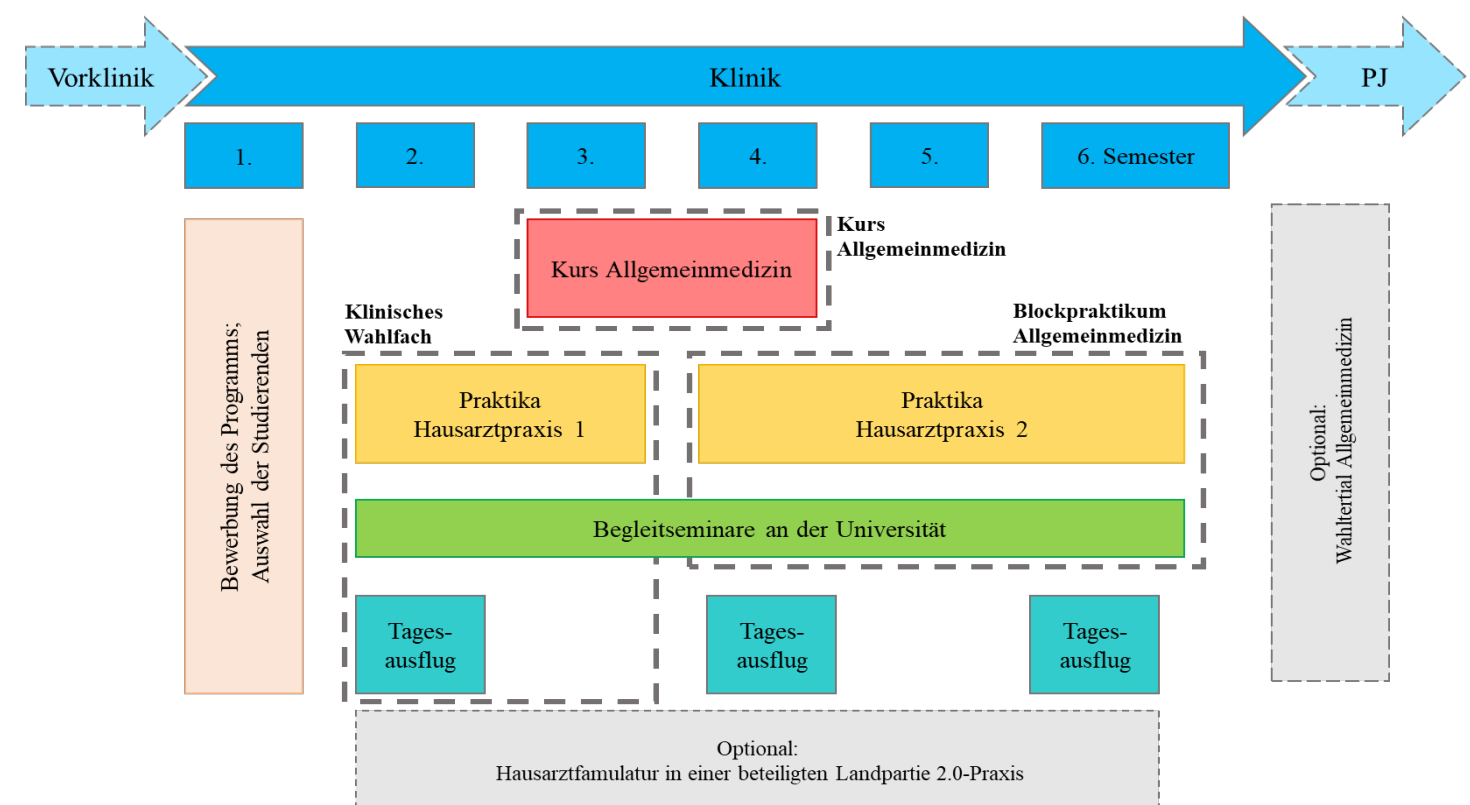

Abbildung 1: Curricularer Ablauf der Landpartie 2.0 im klinischen Studienabschnitt (Quelle: eigene Darstellung).

Kernelement des Programms sind regelmäßige praktische Erfahrungen ( 2 bis 5 Tage pro Semester) in 1:1-Betreuung in ländlich gelegenen Hausarztpraxen. Die Praxisphasen finden stets in der vorlesungsfreien Zeit statt und der konkrete Termin wird individuell zwischen Studierenden und Lehrärzten/innen geplant. Die Mehrheit der Lehrärzte/innen wurde dabei eigens für die „Landpartie 2.0“ neu rekrutiert und vor Beginn der Lehrtätigkeit organisatorisch eingeführt und didaktisch geschult.

Indem die Studierenden innerhalb von fünf Semestern zwei verschiedene Praxen durchlaufen, sollen sie ein möglichst breites Spektrum hausärztlicher Versorgung kennenlernen (z. B. Praxis 1: Einzelpraxis in einer Kommune mit 20.000 Einwohnern; Praxis 2: Gemeinschaftspraxis in einer Kommune mit 2.700 Einwohnern). Um einen 
finanziellen Aufwand für die teilnehmenden Studierenden zu vermeiden, werden Fahrtund Übernachtungskosten sowie eine Verpflegungspauschale durch die beteiligten Landkreise übernommen.

Neben den Praxisphasen finden regelmäßig Seminare am Institut für Allgemeinmedizin statt. Das Themenspektrum ist vielfältig und reicht von Fallbesprechungen, persönlichem Erfahrungsaustausch, Strategien zur Gesprächsführung bis hin zur Besprechung von Therapiestrategien. Aber auch interdisziplinäre Themen (z.B. ambulante Palliativmedizin oder Psychosomatik) sowie wirtschaftliche/organisatorische Aspekte zum Führen einer Praxis sind Gegenstand der Seminarreihe. Bei Bedarf können weitere Themen mit der Studierendengruppe abgestimmt werden.

Der Kurs Allgemeinmedizin erfolgt für die Teilnehmenden der „Landpartie 2.0“ in besonders kleinen Gruppen von max. sieben Studierenden (regulär 8-10 Studierende) in einer geschulten Hausarztpraxis auf dem Land. Der Kurs umfasst 10,5 Zeitstunden und findet im 3. oder 4. klinischen Semester statt. Die Studierenden lernen u. a. typische allgemeinmedizinische Vorgehensweisen und Krankheitsbilder der hausärztlichen Praxis kennen.

Zusätzlich wird den Teilnehmenden im Programm einmal pro Jahr ein Tagesausflug zu innovativen Modellen der Gesundheitsversorgung angeboten. Ziel ist es, das breite Spektrum an Kooperations- und Organisationsformen in der hausärztlichen Versorgung (auch in ländlichen Regionen) aufzuzeigen.

Zur Unterstützung der Studierenden und der Lehrärzte/innen wurde zudem ein eigenes Logbuch erstellt, das über das gesamte Programm hinweg als Dokumentationshilfe und als Grundlage für die inhaltliche Gestaltung der Praktika dient. Das Logbuch bietet die Möglichkeit, Lernziele für die einzelnen Praktika festzuhalten und somit auch einen Überblick über die individuelle Kompetenzentwicklung zu erstellen. Ferner beinhaltet das Logbuch Feedbackbögen, Teilnahmebestätigungen für alle curricular verpflichtenden Veranstaltungen sowie die Bewertungsbögen für die Notenvergabe.

Neben der Erstattung von Fahrt- und Übernachtungskosten erhalten die teilnehmenden Studierenden zudem pro Semester einen Büchergutschein und finanzielle Unterstützung bei der Teilnahme an wissenschaftlichen Kongressen mit allgemeinmedizinischem Bezug. 


\section{Ergebnisse und Ausblick}

Zum Wintersemester 2016/2017 wurde die Landpartie 2.0 den Medizinstudierenden erstmals angeboten. Seitdem konnten 62 Studierende in das Programm aufgenommen werden. ${ }^{1}$

Hinsichtlich der sozio-demografischen Merkmale zeigt sich ein sehr heterogenes Bild innerhalb der bisherigen Teilnehmenden (vgl. Tabelle 1). Das Verhältnis von Frauen (69,4 \%) zu Männern (30,6 \%) ähnelt dabei dem im gesamten Medizinstudium, schwankt jedoch innerhalb der Jahrgänge. Im Durchschnitt sind die Teilnehmenden zum Bewerbungszeitpunkt 24 Jahre alt [Range: 20-48 Jahre] und ältere Bewerber/innen verfügen häufiger über eine abgeschlossene Berufsausbildung. Überraschend ist hingegen, dass ca. die Hälfte aller Studierenden aus Gemeinden mit mehr als 10.000 Einwohnern stammt und somit, der dem Programm zugrundeliegenden Definition, nicht ländlich aufgewachsen ist. Dieses Ergebnis deckt sich jedoch mit den Freitextantworten aus den Bewerbungsbögen. Während einige Bewerber/innen wiederkehrend ihren persönlichen Bezug zum Leben auf dem Land zum Ausdruck bringen, schreiben andere Studierende explizit, dass sie aus einer Großstadt stammen, aber im Rahmen des Programms durchaus die Chance nutzen wollen, andere Lebensstile und womöglich auch eine andere Art der hausärztlichen Medizin kennenlernen zu wollen.

\footnotetext{
${ }^{1}$ Regulär stehen 15 Plätze im ersten klinischen Semester zur Verfügung. In den ersten drei Jahren gingen jedoch weniger Bewerbungen ein als Plätze vorhanden waren. Aus diesem Grund wurde das Programm auch den Studierenden im dritten klinischen Semester angeboten die dann jedoch nur noch die Hausarztpraxisphase 2 und ein Teil der Seminare im Rahmen des Programms absolvieren. Auf diese Weise wurden in den ersten drei Jahren 16, 15 und 14 Plätze belegt. Im Wintersemester 2019/2020 lag die Bewerberzahl mit 26 erstmals über den zur Verfügung stehenden Plätzen. Aufgrund der sehr guten Bewerberlage und den kapazitiven Möglichkeiten wurden zwei Studierende mehr aufgenommen.
} 
Tabelle 1: Sozio-demografische Angaben zum Bewerbungszeitpunkt (ca. November eines jeden Jahres); (Quelle: eigene Angaben).

\begin{tabular}{|c|c|c|c|c|c|c|c|c|}
\hline & \multicolumn{2}{|l|}{ Semester } & \multicolumn{2}{|l|}{ Geschlecht } & \multirow[b]{2}{*}{$\begin{array}{l}\text { Alters- } \\
\text { durchschnitt } \\
\text { in Jahren } \\
\text { [Altersspanne] }\end{array}$} & \multicolumn{2}{|c|}{ Herkunft (Anzahl) } & \multirow[b]{2}{*}{$\begin{array}{l}\text { Abgeschlossene } \\
\text { Berufsausbildung } \\
\text { (Anzahl) }\end{array}$} \\
\hline & $\begin{array}{l}\text { Erstes } \\
\text { klinisches } \\
\text { Semester }\end{array}$ & $\begin{array}{l}\text { Drittes } \\
\text { klinisches } \\
\text { Semester }\end{array}$ & Männlich & Weiblich & & $\begin{array}{l}\text { Ländlich } \\
\text { bis } 10.000 \\
\text { Einwohner }\end{array}$ & $\begin{array}{l}\text { Städtisch } \\
>10.000 \\
\text { Einwohner }\end{array}$ & \\
\hline $\begin{array}{l}\text { Kohorte } \\
2016 / 17\end{array}$ & 11 & 5 & 7 & 9 & $\begin{array}{c}26 \\
{[20-48]}\end{array}$ & 6 & 10 & 4 \\
\hline $\begin{array}{l}\text { Kohorte } \\
2017 / 18\end{array}$ & 12 & 3 & 3 & 12 & $\begin{array}{c}22 \\
{[20-25]}\end{array}$ & 4 & 11 & 1 \\
\hline \multirow{2}{*}{$\begin{array}{l}\text { Kohorte } \\
2018 / 19\end{array}$} & \multirow[t]{2}{*}{13} & \multirow[t]{2}{*}{1} & \multirow{2}{*}{7} & \multirow{2}{*}{7} & \multirow{2}{*}{$\begin{array}{c}27 \\
{[21-37]}\end{array}$} & 8 & 5 & \multirow{2}{*}{9} \\
\hline & & & & & & \multicolumn{2}{|c|}{1 ,weiß nicht" } & \\
\hline $\begin{array}{l}\text { Kohorte } \\
2019 / 20\end{array}$ & 17 & 0 & 2 & 15 & $\begin{array}{c}23 \\
{[20-29]}\end{array}$ & 12 & 5 & 3 \\
\hline \multirow{2}{*}{ Gesamt } & 53 & 9 & 19 & 43 & \multirow{2}{*}{$\begin{array}{c}24 \\
{[20-48]}\end{array}$} & \multirow{2}{*}{30} & \multirow{2}{*}{31} & \multirow{2}{*}{17} \\
\hline & \multicolumn{4}{|c|}{62} & & & & \\
\hline
\end{tabular}

Die ersten Evaluationsergebnisse beziehen sich auf die Praxisphasen, die begleitende Seminarreihe sowie den jährlichen Tagesausflug, Stand Ende Sommersemester 2019 (vgl. Abb. 2). Langfristig sollen darüber hinaus in einer Verbleibstudie Effekte auf die Motivation für eine hausärztliche Tätigkeit (auf dem Land) sowie Karriereverläufe abgebildet werden.

Bisher zeigt sich eine sehr hohe Zufriedenheit bezüglich der praktischen Programmbestandteile. Knapp $95 \%$ berichten ferner von einem subjektiven Wissenszuwachs und stufen die Praktika als nützlichen Abschnitt ihrer ärztlichen Ausbildung ein. Im Durchschnitt werden die jeweiligen Praktika mit der Schulnote 1,51 bewertet $(\mathrm{N}=37)$. Auch bezüglich der Seminare wird deutlich, dass die Ansprüche der Studierenden an Organisation und Inhalt nahezu vollständig erfüllt werden. Eine Vermittlung relevanter Inhalte und einen Wissenszuwachs bestätigten 93\% der Befragten. Das Engagement der Dozierenden und deren strukturierte Darstellung des Lehrstoffs wurden zudem bis auf eine Nennung durchgehend positiv bewertet.

Seit Beginn der „Landpartie 2.0“ haben bisher drei Tagesausflüge zu unterschiedlichen innovativen Gesundheitsmodellen in jeweils einem der beteiligten Landkreise stattgefunden. Die Evaluationsergebnisse zeigen, dass die Erwartungen der Studierenden durchgehend erfüllt wurden. Große Zustimmung fand die Organisation rund um den 
Ausflug. Bei 91\% der Studierenden konnte der Tagesausflug zu einem Wissenszuwachs beitragen und 94\% fühlten sich über neue berufliche Möglichkeiten informiert. 98\% würden den Tagesausflug weiterempfehlen. Im Mittel wurde der Tagesausflug mit der Schulnote 1,43 bewertet $(\mathrm{N}=47)$.

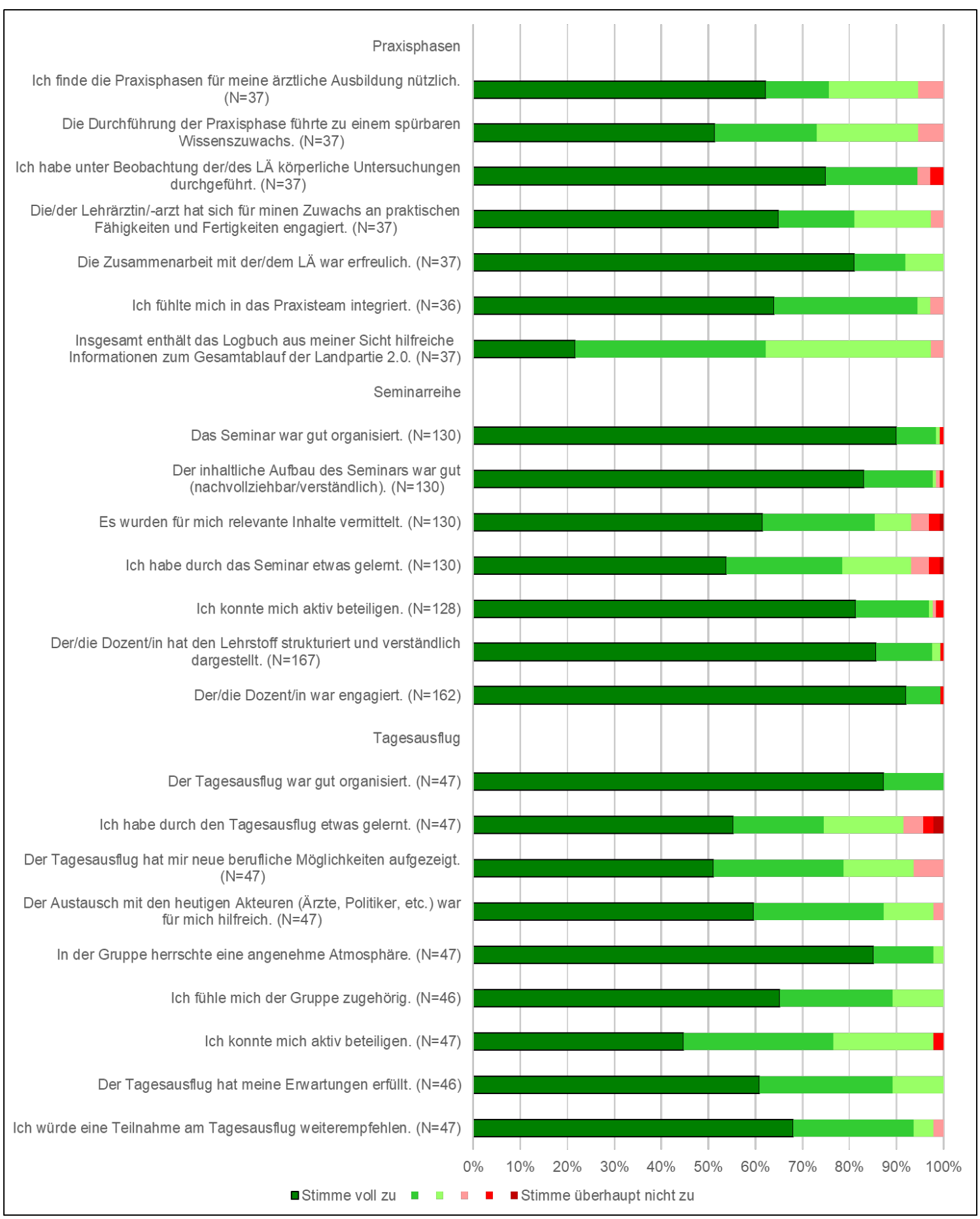

Abbildung 2: Evaluationsergebnisse der Praxisphasen, der Seminarreihe und des Tagesausflugs bis Sommersemester 2019 (Quelle: eigene Darstellung). 


\subsection{Gesamtdiskussion}

In mehreren Arbeitsschritten konnte mittels einer Methodentriangulation (,,betweenmethod") ein Schwerpunktprogramm, das die Allgemeinmedizin in ländlichen Regionen bereits im Studienverlauf fördern soll, konzeptionell entwickelt und anschließend erfolgreich in das bestehende Curriculum implementiert werden.

Im ersten Schritt erfolgte eine Bestandsaufnahme zu etablierten bzw. geplanten Angeboten an allen medizinischen Fakultäten in Deutschland. Das gewählte methodische Vorgehen hat dazu geführt, dass von Beginn an Möglichkeiten und Grenzen eines „Landarztprogramms“ erkannt und berücksichtigt werden konnten.

Durch die umfassende Befragung aller medizinischen Fakultäten wurden Programme identifiziert, von denen einzelne Bestandteile in die Erarbeitung des eigenen Angebots einfließen konnten. Neben der inhaltlichen Gestaltung wurde den Entwicklern/innen der „Landpartie 2.0" aber auch verdeutlicht, welche formellen Hürden es zu beachten gilt, wenn die Einführung eines breit angelegten, praxisorientierten Angebots dauerhaft gelingen soll. Insbesondere die langfristige Absicherung finanzieller Ressourcen und der damit verbundenen, personellen Ausstattung kommt eine, wenn vielleicht logische, aber oftmals nicht gegebene, wesentliche Rolle zu. Folglich war und ist es ein großes Anliegen des eigenen Vorhabens, die finanziell benötigten Mittel durch Eigenakquise abzusichern. Um Programme wie die „Landpartie 2.0“ auch an anderen medizinischen Fakultäten umsetzen zu können, sollten bundesweit konkrete Schritte erfolgen, um nachhaltig finanzielle Ressourcen zur Verfügung zu stellen.

Im Rahmen des zweiten Schritts - der webbasierten Befragung der Medizinstudierenden - konnten auch aufgrund der sehr guten Rücklaufquote im Rahmen einer webbasierten Befragung $(=28,7 \%$ ) weitere konkrete Hinweise und Anregungen für die organisatorische sowie inhaltliche Gestaltung der einzelnen Bestandteile des Programms gewonnen werden. So wurde z.B. definiert, dass das Lehrangebot erst ab dem klinischen Studienabschnitt startet und neben dem zentralen Element eines kontinuierlichen Praxisbezugs auch Themen zur Praxisführung und -management in einer begleitenden Seminarreihe integriert sein sollten. Darüber hinaus ist anzunehmen, dass die Beteiligung der Studierenden am Gestaltungsprozess der „Landpartie 2.0“ zu einer höheren Akzeptanz des Programmes geführt hat. Gleichzeitig muss an dieser Stelle auch berücksichtigt werden, dass an der Befragung wahrscheinlich vorrangig Studierende 
teilgenommen haben, die der Allgemeinmedizin positiv gegenüberstehen und womöglich ein Interesse an einer spezialisierten Ausbildung im Fach haben. Die Ergebnisse könnten somit dem Meinungsbild einer selbstselektierten Gruppe entsprechen. Da jedoch auch 125 Studierende an der Befragung teilgenommen haben, die nach eigener Aussage kein Interesse am Fachgebiet zeigen, kann davon ausgegangen werden, dass die beschriebene Limitation nur eingeschränkt zutrifft.

Im Ergebnis handelt es sich bei der „Landpartie 2.0“ um ein freiwilliges, longitudinales Schwerpunktprogramm im klinischen Studienabschnitt, das über drei JahrgangsKohorten hinweg insgesamt ca. 45 interessierte Studierende aufnehmen kann. Das Förderangebot beinhaltet verschiedene Komponenten (,regelmäßige Praxisphasen“, „Seminarreihe“, ,jährlicher Tagesausflug“, „Kurs Allgemeinmedizin“), die inhaltlich und organisatorisch ineinandergreifen und so ein komplexes sowie praxisorientiertes Gesamtkonzept ergeben.

Erste Evaluationsergebnisse zur Zufriedenheit bestätigen, dass das Schwerpunktprogramm die Wünsche und Anforderungen der Studierenden erfüllt. In Zukunft sollen aber insbesondere langfristig angelegte Verbleibstudien zeigen, ob eine Teilnahme am Angebot das Interesse an der Allgemeinmedizin erhöht und ob die Wahrscheinlichkeit steigt, dass sich Teilnehmende gegenüber Nicht-Teilnehmenden mit ähnlicher Interessenslage eher für ein PJ-Wahltertial und/oder die Weiterbildung Allgemeinmedizin bzw. eine hausärztliche Tätigkeit (in ländlichen Regionen) entscheiden. Fortlaufende Prozess- sowie Ergebnisevaluationen müssen hier in Zukunft sowohl die Qualität als auch die Zielsetzung der „Landpartie 2.0“ überprüfen. Dabei ist zu beachten, dass es sich bei den Teilnehmenden a priori um Studierende handelt, die ein grundlegendes, wenn nicht gar gesteigertes Interesse an der hausärztlichen Versorgung haben und somit eine größere Wahrscheinlichkeit bestehen kann, einen allgemeinmedizinischen Karriereweg einzuschlagen. Dieser Selektionsbias muss somit in der Evaluation bzw. im methodischen Vorgehen berücksichtig werden.

Aus der beschriebenen inhaltlichen sowie strukturellen Komplexität des Programms und den ersten positiven Evaluationsergebnissen ergibt sich, dass die „Landpartie 2.0“ nicht nur den Forderungen nach mehr allgemeinmedizinischer Praxis im Studium nachkommt ${ }^{24,25}$, sondern ebenfalls das Potential besitzt, Studierende für das Fach der Allgemeinmedizin nachhaltig $\mathrm{zu}$ begeistern und im Sinne einer hausärztlichen Karriereplanung positiv zu beeinflussen. Damit stellt das Programm eine Maßnahme dar, 
dem zunehmenden Mangel an Hausärzten/innen insbesondere im ländlichen Raum zu begegnen und gleichzeitig die Attraktivität und Bedeutsamkeit dieser Berufsgruppe zu steigern.

Der freiwillige Charakter des Schwerpunktprogramms ermöglicht es den Teilnehmenden zudem, in eine allgemeinmedizinische Tätigkeit abseits der Metropolen „hinein zu schnuppern" ohne langfristige und bindende Verpflichtungen einzugehen, wie beispielsweise eine obligatorische Niederlassung im ländlichen Raum. ${ }^{37,38}$ Ein Angebot, wie die „Landpartie 2.0“ stellt somit eine alternative Möglichkeit zu der in einigen Bundesländern bereits eingeführten bzw. in Hessen aktuell diskutierten „Landarztquote“ ${ }^{\text {(2 }}$ dar. Denkbar wäre jedoch, dass ein Programm wie die „Landpartie 2.0“ mit der Einführung einer „Landarztquote“ gekoppelt wird. Studierende, die ihren Studienplatz über die Quotenregelung erhalten, nehmen so an einem strukturierten und longitudinalen Schwerpunktprogramm teil, das sie sowohl fachlich als auch mental auf ihre spätere Tätigkeit als Hausarzt/ärztin vorbereitet. Somit könnte auch der Einschränkung entgegengewirkt werden, dass mit dem Programm der „Landpartie 2.0“ voraussichtlich lediglich Studierende angesprochen werden, die, wie beschrieben, in der Regel ein grundlegendes Interesse an der Allgemeinmedizin haben.

Dennoch sollte bei weiteren positiven Evaluationsergebnissen über die Etablierung eines Schwerpunktprogramms an allen medizinischen Standorten in Deutschland nachgedacht werden, um so einen aktiven und effektiven Beitrag in der hausärztlichen Nachwuchssicherung zu leisten. Universitätsspezifische Besonderheiten und Schwerpunkte könnten dabei berücksichtigt werden und dennoch wäre ein gegenseitiges „voneinander lernen“ möglich. Ein durch den Bund finanziertes, flächendeckendes Ausrollen des Programms bzw. dessen Verankerung in jedes Regelcurriculum würde zudem für Teilnehmende den Vorteil bieten, dass nach einem Studienstandortwechsel, die Programminhalte nahtlos fortgeführt werden könnten. Ferner wäre eine große, über mehrere Standorte hinweg angelegte Evaluationsstudie möglich, die zur Qualität und Aussagekraft der Ergebnisse beitragen würde.

\footnotetext{
${ }^{2}$ Bis zu 10 Prozent der Medizinstudienplätze können vorab an Bewerberinnen/Bewerber vergeben werden, die sich vertraglich dazu verpflichten, nach Ihrem Studium und der fachärztlichen Weiterbildung in der Allgemeinmedizin für bis zu zehn Jahre in der hausärztlichen Versorgung in unterversorgten bzw. durch Unterversorgung bedrohten (ländlichen) Regionen tätig zu sein. ${ }^{39}$
} 
Insgesamt handelt es sich nach dem Wissen der Autorin bei der „Landpartie 2.0“, um das in Deutschland erste, freiwillige, breit angelegte und über mehrere Semester hinweg konzipierte Schwerpunktprogramm, das explizit auf die Förderung der Allgemeinmedizin in ländlichen Regionen zielt. Durch den breiten methodischen Ansatz und die hohen Rücklaufquoten, bezogen auf die jeweilige Methodik, konnten von Beginn an BestPractice-Beispiele, die Anforderungen und Wünsche der Zielgruppe selbst sowie die geltenden curricularen bzw. rechtlichen Rahmenbedingungen in Deutschland berücksichtigt werden, die so letztlich zu der erfolgreichen, konzeptionellen Gestaltung eines fachbereichsweiten Lehrangebots, welches es Medizinstudierenden ermöglicht, Allgemeinmedizin im ländlichen Raum kennenzulernen, führten. Die aktuell steigenden Bewerberzahlen lassen zudem vermuten, dass die „Landpartie 2.0“ nicht nur eine zunehmende Bekanntheit unter den Studierenden erlangt, sondern auch deren Interesse an einer akzentuierten und praxisnahen Ausbildung im klinischen Studienabschnitt trifft.

\subsection{Schlussfolgerung}

Das fakultative Programm der „Landpartie 2.0“ konnte erfolgreich konzipiert und in den klinischen Studienabschnitt integriert werden. Das gewählte methodische Vorgehen hat dazu beigetragen, von Beginn an Möglichkeiten und Grenzen eines solchen Angebots zu erkennen und in dessen konzeptioneller Entwicklung zu berücksichtigen.

Erste Evaluationsergebnisse verweisen darauf, dass die „Landpartie 2.0“ den Bedarf der Studierenden erfüllt, einen wertvollen Beitrag in der medizinischen Ausbildung leistet und trotz ihres ländlichen Fokus auch Studierende aus urbanen Regionen anspricht. Insgesamt kann das entwickelte Schwerpunktprogramm aus Sicht der Autorin aufgrund seines longitudinalen und umfassenden Charakters den weiteren Ausbildungs- und Berufsweg von Medizinstudierenden im Sinne einer hausärztlichen Tätigkeit positiv beeinflussen. 


\section{Literaturverzeichnis}

1. Kassenärztliche Bundesvereinigung. Ärztemangel. https://www.kbv.de/html/themen_1076.php. Accessed June 2, 2020.

2. Sachverständigenrat zur Begutachtung der Entwicklung im Gesundheitswesen. Sondergutachten 2009. Koordination und Integration - Gesundheitsversorgung in einer Gesellschaft des längeren Lebens. Bonn/Berlin. Ziffern 636-728; 2009. http://dip21.bundestag.de/dip21/btd/16/137/1613770.pdf. Accessed June 2, 2020.

3. Kassenärztliche Bundesvereinigung. Gesundheitsdaten. https://gesundheitsdaten.kbv.de/cms/html/17075.php. Accessed June 2, 2020.

4. Bundesärztekammer. Ergebnisse der Ärztestatistik zum 31. Dezember 2014. http:/www.bundesaerztekammer.de/fileadmin/user_upload/downloads/pdfOrdner/Statistik2014/Stat14AbbTab.pdf. Accessed June, 23, 2020.

5. Heinz A, Jacob R. Medizinstudenten und ihre Berufsperspektiven. In welcher Facharztrichtung, wo und wie wollen sie arbeiten? Bundesgesundheitsbl. 2012;55:245-253.

6. Sachverständigenrat zur Begutachtung der Entwicklung im Gesundheitswesen. Bedarfsgerechte Versorgung. Perspektiven für ländliche Regionen und ausgewählte Leistungsbereiche. Gutachten 2014. Ziffer 481. Bonn/Berlin. https://www.svr-gesundheit.de/fileadmin/user_upload/Gutachten/2014/SVRGutachten_2014_Langfassung.pdf. Accessed April 16, 2020.

7. Samos FA, Heise M, Fuchs S, Mittmann S, Bauer A, Klement A. A pilot phase evaluation of the elective general pracitice class: results of student surveys of the first two years. GMS J Med Educ. 2017;34(1) doi: 10.3205/zma001081.

8. Deutsch T, Hönigschmid P, Frese T, Sandholzer H. Early community-based family practice elective positively influences medical students' career considerations - a pre-post-comparison. BMC Fam Pract. 2013;2:14-24. doi: 10.1186/1471-2296-14-24.

9. Rabinowitz HK, Diamond JJ, Markham FW, Wortman JR. Medical school programs to increase the rural physician supply: a systematic review and projected 
impact of widespread replication. Acad Med. 2008;83(3):235-243. doi:

10.1097/ACM.0b013e318163789b.

10. Krupa LK, Chan BT. Canadian rural family medicine training programs: growth and variation in recruitment. Can Fam Physician. 2005;51:852-53.

11. Eley DS, Synnott R, Baker PG, Chater AB. A decade of Australian rural clinical school graduates - where are they and why? Rural and Remote Health.

2012;12:1937. http://www.rrh.org.au/publishedarticles/article_print_1937.pdf. Accessed May 23, 2020.

12. Strasser RP, Lanphear JH, McCready WG, Topps MH, Hunt DD, Matte MC. Canada's new medical school. Acad Med. 2009;84:1459-1464 15.

13. The University of Western Australia. Rural Clinical School of WA. https://www.uwa.edu.au/health/Schools/medical-school/rural-clinical-school. Accessed April 5, 2020.

14. Rabinowitz HK, Petterson S, Boulger JG, Matthew L, Diamond JJ, Markham FW, et al. Medical School Rural Programs: a comparison with international medical graduates in addressing state-level rural family physician and primary care supply. Acad Med. 2012;87(4):488-492. doi: 10.1097/ACM.0b013e3182488b19.

15. Wendling AL, Phillips J, Short W, Fahey C, Mavis B. Thirty Years Training Rural Physicians: Outcomes From the Michigan State University College of Human Medicine Rural Physician Program. Acad Med. 2016;91(1):113-119. doi: 10.1097/ACM.0000000000000885.

16. Rabinowitz HK, Diamond JJ, Markham FW, Santana AJ. Retention of Rural Family Physicians After 20-25 Years: Outcomes of a Comprehensive Medical School Rural Program. J Am Board Fam Med. 2013;26(1):24-27. doi: 10.3122/jabfm.2013.01.120122.

17. Blozik E, Ehrhardt M, Scherer M. Förderung des allgemeinmedizinischen Nachwuchses. Initiativen in der universitären Ausbildung von Medizinstudierenden. Bundesgesundheitsbl 2014;57(7):892-902. doi.org/10.1007/s00103-014-1984-6.

18. Steger T, Langosch C, Klement A, Onnasch J. „Klasse Allgemeinmedizin“: ein Lehrkonzept für zukünftige Landärzte. Z Allg Med. 2012;88(6):264-267. 
19. Herrmann M, Hänel P, Jansen E. Landärztliche Ausbildung zur Reduzierung der medizinischen Unterversorgung auf dem Land - Erfahrungen, Chancen, Widrigkeiten. In Brunnett R et al., ed. Jahrbuch für Kritische Medizin und Gesundheitswissenschaften 50. Hamburg: Argument Verlag; 2014:144-172.

20. Schäfer H, Mangold B, Paulitsch M, Gerlach FM. Landpartie Fulda - ein Projekt zur Förderung des hausärztlichen Nachwuchses im ländlichen Raum. Z Allg Med 2015;91:260-263.

21. Meurer LN. Influence of medical school curriculum on primary care specialty choice: analysis and synthesis of the literature. Acad Med. 1995;70(5):388-397. doi:10.1097/00001888-199505000-00015.

22. Pfarrwaller E, Sommer J, Chung C, et al. Impact of interventions to increase the proportion of medical students choosing a primary care career: A systematic review. J Gen Intern Med. 2015;30(9):1349-1358. doi:10.1007/s11606-015-33729.

23. Bundesvertretung der Medizinstudierenden in Deutschland (bvmd). Longitudinale Einbindung der Allgemeinmedizin in das Medizinstudium. Berlin; 2014. https://www.bvmd.de/fileadmin/redaktion/Positionspapiere/Positionspapier_201404-26_Longit.-Einbindung-der-Allgemeinmed-in-Medizinstudium.pdf. Accessed April 30, 2020.

24. Huenges B, Gulich M, Böhme K, Fehr F, Streitlein-Böhme I, Rüttermann V, Baum E, Niebling WB, Rusche H. Recommendations for undergraduate training in the primary care sector - position paper of the GMA-Primary Care Committee. GMS Z Med Ausbild. 2014;31(3):Doc35. doi: 10.3205/zma000927.

25. Flick U. Triangulation. In Oelerich G, Otto HU, ed. Empirische Forschung und Soziale Arbeit. Wiesbaden, VS Verlag für Sozialwissenschaften. 2011:323-328. doi: 10.1007/978-3-531-92708-4_23.

26. MacKean P, Gutkin C. Fewer medical students selecting family medicine. Can Fam Physician 2003;49:408-409. 
27. Bayerisches Ärzteblatt. Hausarztleben im Bayerischen Wald. https://www.bayerischesaerzteblatt.de/inhalte/details/news/detail/News/hausarztleben-im-bayerischenwald.html. Accessed September 4; 2019.

28. Nohl-Deryk P. Landarzt-Famulaturen: Heiss begehrt. Ärzteblatt https://www.aerzteblatt.de/archiv/172217/Landarzt-Famulaturen-Heiss-begehrt. Accessed September 4; 2019.

29. Barthen L, Beig I, Sennekamp M, Gerlach FM, Erler A, Ravens-Taueber G. Raus aufs Land während des Medizinstudiums: Eine Übersicht zu bestehenden und geplanten allgemeinmedizinischen Förderangeboten. Z Allg Med. 2016;92(11):448-445. doi:10.3238/zfa.2016.0448-0455.

30. Yengo-Kahn AM, Baker CE, Lomis KD. Medical students' perspectives on implementing curriculum change at one institution. Acad Med. 2017;92(4):455461. doi:10.1097/ACM.0000000000001569.

31. Atkins KM, Roberts AE, Cochran N. How medical students can bring about curricular change. Acad Med. 1998;73(11):1173-76.

32. Barthen L, Ravens-Taeuber G, Paulitsch MA, Gerlach FM, Sennekamp M. How can General Practice be incorporated longitudinally in medical studies? Students' views on the development of a new rural health program. GMS J Med Educ. 2018;35(3):Doc42. doi:10.3205/zma001188.

33. Jacob R, Kopp J, Schultz S. Berufsmonitoring Medizinstudenten 2014: Ergebnisse einer bundesweiten Befragung. Berlin; 2015. http://www.kbv.de/media/sp/2015_04_08_Berufsmonitoring_2014_web.pdf. Accessed May 13, 2020.

34. Seeger L, Becker N, Ravens-Taeuber G, Sennekamp M, Gerlach FM. "Landpartie 2.0" - Conceptual development and implementation of a longitudinal priority program to promote family medicine in rural areas. GMS J Med Educ. 2020;37(3):Doc29. doi: 10.3205/zma001322. 
35. Deutsch T, Lippmann S, Frese T, Sandholzer H. Who wants to become a general practitioner? Student and curriculum factors associated with choosing a GP career - a multivariable analysis with particular consideration of practice-orientated GP courses. Scand J Prim Health Care. 2015;33(1):47-53. doi:10.3109/02813432.2015.1020661.

36. Viscomi M, Larkins S, Gupta TS. Recruitment and retention of general practitioners in rural Canada and Australia: a review of the literature. Can J Rural Med. 2013;18(1):13-23.

37. Sachsen.de. Sächsisches Hausarztstipendium für Medizinstudierende. 2020. https://www.gesunde.sachsen.de/stipendium-medizin.html. Accessed May 30, 2020 .

38. Bayerisches Landesamt für Gesundheit und Lebensmittelsicherheit. Stipendium für Medizinstudierende. 2019. https://www.lgl.bayern.de/gesundheit/bayerische_gesundheitsagentur/foerderprog ramme/stipendien/index.htm. Accessed June 15, 2020.

39. Bundesministerium für Bildung und Forschung. Masterplan Medizinstudium 2020. 2019. https://www.bmbf.de/de/masterplan-medizinstudium-20204024.html_Accessed June 15, 2020. 


\section{Übersicht der veröffentlichten Publikationen}

\section{Erste Publikation}

Barthen L, Beig I, Sennekamp M, Gerlach FM, Erler A, Ravens-Taueber G. Raus auf's Land während des Medizinstudiums: Eine Übersicht $\mathrm{zu}$ bestehenden und geplanten allgemeinmedizinischen Förderangeboten. $Z$ Allg Med. 2016;92(11):448-445. doi: 10.3238/zfa.2016.0448-0455.

\section{Zweite Publikation}

Barthen L, Ravens-Taeuber G, Paulitsch MA, Gerlach FM, Sennekamp M. How can General Practice be incorporated longitudinally in medical studies? Students' views on the development of a new rural health program. GMS J Med Educ. 2018;35(3):Doc42. doi: $10.3205 / \mathrm{zma} 001188$.

\section{Dritte Publikation}

Seeger L, Becker N, Ravens-Taeuber G, Sennekamp M, Gerlach FM. "Landpartie 2.0" Conceptual development and implementation of a longitudinal priority program to promote family medicine in rural areas. GMS J Med Educ. 2020;37(3):Doc29. doi: $10.3205 / \mathrm{zma001322.}$

\section{Veröffentlichte Publikationen}




\title{
Raus aufs Land während des Medizinstudiums
}

\author{
Eine Übersicht zu bestehenden und geplanten \\ allgemeinmedizinischen Förderangeboten
}

\section{Get Out to the Countryside during Medical Education}

\author{
An Overview of all Established and Proposed Rural Medical Programs \\ Linda Barthen, Inga Beig, Monika Sennekamp, Ferdinand M. Gerlach, Antje Erler, Gisela Ravens-Taeuber
}

\begin{abstract}
Hintergrund: Seit Jahren leidet die hausärztliche Versorgung in Deutschland unter einem akuten Nachwuchsproblem. Flächenstaaten, wie die USA oder Australien, die einer ähnlichen Problematik ausgesetzt sind, haben als Gegenmaßnahme Schwerpunktprogramme entwickelt, die eine spätere allgemeinmedizinische Tätigkeit im ländlichen Raum bereits während des Studiums fördern. In Deutschland gibt es erste Initiativen, die sich ebenfalls dieser Thematik annehmen. Eine umfassende Übersicht zu bestehenden und geplanten universitären Angeboten in Deutschland fehlt bisher. Methoden: Mittels Internetrecherche und telefonischen Expertengesprächen wurde eine Übersicht bestehender und zukünftig geplanter Angebote erstellt. Hierzu wurden alle 37 allgemeinmedizinischen Einrichtungen an deutschen Fakultäten kontaktiert.

Ergebnisse: 31 Einrichtungen nahmen an der Untersuchung teil (83,8 \%). An 12 Fakultäten konnten 13 relevante Angebote identifiziert werden. Drei davon weisen einen longitudinalen Charakter auf bzw. dauern mindestens zwei Semester. Die meisten Veranstaltungen richten sich an eine geringe Anzahl von Studierenden pro Durchgang und/oder Jahr. Die Zahl der Angebote ist in den letzten Jahren stetig gestiegen.
\end{abstract}

Schlussfolgerungen: Die Förderung einer späteren hausärztlichen Tätigkeit in ländlichen Regionen während des Studiums ist von zunehmender Bedeutung für die Universitäten. Beginn, Dauer und Ausgestaltung der Ansätze unterscheiden sich zum Teil deutlich voneinander, erreichen aber insgesamt nicht die Intensität von etablierten Programmen im Ausland. Welche der Ansätze einen positiven Beitrag zur Steigerung der Zahl der Allgemeinmediziner im ländlichen Raum leisten können, müssen Evaluationsergebnisse zeigen. Die erstellte Übersicht kann allen Universitäten, die sich dieser Thematik in Zukunft annehmen wollen, als Orientierungs- und Vernetzungshilfe dienen.

Schlüsselwörter: Übersichtsarbeit; Medizinstudium; Allgemeinmedizin; Landarztprogramme
Background: Primary health care in Germany is having enormous problems recruiting young doctors. The United States and Australia, who are facing similar problems, have developed physician shortage area programs as a countermeasure. These promote primary health care in rural areas during medical education. In Germany, several initiatives dealing with the same topic have evolved. However, no overview of established and proposed rural medical programs currently exists.

Methods: An overview outlining all established and proposed rural medical programs at German medical faculties was developed by searching websites and conducting expert discussions by telephone. For this purpose, all 37 institutes of family medicine in Germany were contacted.

Results: 31 institutes participated in the study (83,8 \%). 13 rural medical programs were identified at 12 faculties. Three of them are longitudinal, meaning they last at least two semesters. In most cases, the courses are restricted and are only designed for a small number of students per year or program. The number of programs has increased steadily over the past years.

Conclusions: The importance of promoting primary health care in rural areas during undergraduate medical education increases. Start, duration and design of the identified programs vary considerably. All in all, they are not as intensive as the well-established programs in other countries. Evaluations of the programs are required to show which of them have had a positive influence on the number of family practitioners in rural areas. All universities interested in the topic can use the overview as a source of orientation and encouragement to get in contact with each other.

Keywords: overview; medical education; family medicine; rural area programs 


\section{Hintergrund}

Die Sicherstellung der hausärztlichen Versorgung in ländlichen Regionen ist in Deutschland zunehmend gefährdet $[1,2]$. Dafür ursächlich ist neben einem steigenden medizinischen Versorgungsbedarf (aufgrund des demografischen Wandels und der damit einhergehenden Zunahme von älteren, chronisch kranken und multimorbiden Patienten*) auch ein anhaltender Nachwuchsmangel an jungen Allgemeinmedizinern. Von den insgesamt 12.231 Facharztanerkennungen im Jahr 2015 entfielen lediglich 10,9 \% (1.337) auf Fachärzte für Allgemeinmedizin [3]. Im Jahr 1993 lag deren Anteil noch bei 18,8 \% [4]. Erschwert wird die Situation dadurch, dass sich die Mehrheit der jungen, angehenden Allgemeinmediziner eine Tätigkeit in urbanisierten und infrastrukturell attraktiven Regionen wünscht [5].

Dieser Entwicklung sehen sich auch andere Industriestaaten dieser Welt ausgesetzt. So stellt die Sicherung der primärärztlichen Versorgung in großen Flächenländern wie den USA, Kanada oder Australien schon lange eine besondere Herausforderung dar [6-9]. Ein Lösungsansatz ist dabei die gezielte Ausund Weiterbildung sowie Rekrutierung von sogenannten „rural health doctors". Hierbei werden Mediziner qualifiziert, die nach ihrem Studienabschluss in einer ländlichen Region tätig werden sollen [10-13]. Neben einzelnen Studiengängen existieren zum Teil auch Universitäten, die sich ausschließlich auf die Aus- und Weiterbildung von Landärzten spezialisiert haben [14, 15]. Evaluationsergebnisse zeigen, dass die Wahrscheinlichkeit, als Hausarzt im ländlichen Raum tätig zu werden, bei Teilnehmern von Schwerpunktprogrammen und Schwerpunktstudiengängen höher ist als bei Studierenden, die einen regulären Studiengang absolvieren $[16,17]$.

In Deutschland existieren im Hinblick auf Umfang und Intensität keine vergleichbaren Ansätze. Gleichwohl scheint das Thema der Förderung des allgemeinmedizinischen Nachwuchses für ländliche Regionen zunehmende Aufmerksamkeit an den Universitäten zu erhalten [18-21]. Auch auf politischer Ebene gewinnt das Thema an Brisanz. So wird beispielsweise die Einführung einer Landarztquote im Rahmen des Masterplans Medizinstudium 2020 kontrovers diskutiert [22].

Einen systematischen Überblick, wie viele Angebote zur Stärkung der Allgemeinmedizin im ländlichen Raum es deutschlandweit an den Universitäten bereits gibt und wie diese inhaltlich strukturiert sind, existiert bisher nicht. Um den aktuellen Stand zu erfassen und zukünftigen Initiativen eine Übersicht zu bieten, soll die vorliegende Untersuchung die Frage beantworten, welche Angebote zur Förderung der Allgemeinmedizin im ländlichen Raum während des Studiums an deutschen medizinischen Fakultäten bereits existieren oder derzeit in Planung sind.

\section{Methoden}

Um die Forschungsfrage zu beantworten wurden eine Internetrecherche durchgeführt, Kontakt mit Verantwortlichen bereits bekannter Förderangebote aufgenommen sowie Telefongespräche mit Mitarbeitern von allgemeinmedizinischen Abteilungen, Instituten und Lehrbereichen in Deutschland geführt. Eingeschlossen wurden Lehrangebote, die es den Studierenden ermöglichen, eine hausärztliche Tätigkeit in einer ländlichen Region kennenzulernen, die als solches Angebot explizit beworben werden und somit für die Studierenden als gezieltes Schwerpunktprogramm erkennbar sind.

Ausgeschlossen wurden Angebote, die ausschließlich auf einer Bezuschussung/Erstattung der Anfahrts- und Übernachtungskosten beruhen. Auch Programme, die keine weiteren fördernden Faktoren beinhalten, wie begleitende Seminare, didaktische Praxisschulungen, ein für das Praktikum eigens entwickeltes Curriculum oder das gezielte Kennenlernen der Region durch begleitende Freizeitangebote wurden nicht berücksichtigt. Die Möglichkeit einer rein zufälligen Einteilung in eine Landpraxis während einer Praxisphase war ebenfalls ein Ausschlusskriterium.

\section{Datenerhebung}

$\mathrm{Zu}$ Beginn wurden die Homepages aller 37 Einrichtungen (Abteilungen/Institute/Lehrbereiche) nach allgemeinmedizinischen Förderprogrammen auf dem Land durchsucht. Gleichzeitig wurden Vertreter bereits bekannter Programme telefonisch kontaktiert $(\mathrm{n}=3)$. In einem weiteren Schritt wurden alle** übrigen allgemeinmedizinischen Einrichtungen personalisiert per E-Mail angeschrieben $(\mathrm{n}=33)$. In dem Schreiben wurde zunächst das eigene Forschungsvorhaben kurz skizziert und abhängig von der vorangegangenen Onlinerecherche gefragt, ob es ein Angebot gibt bzw. ein solches in Planung ist, das der Förderung der Allgemeinmedizin im ländlichen Raum während des Studiums dient. Nach einem Monat wurde eine Erinnerungs-E-Mail verschickt.

Falls bereits Angebote existierten, wurde die Kontaktperson bzw. ein Ansprechpartner um ein telefonisches Expertengespräch gebeten. Die Recherchestrategie und der Prozess der Kontaktaufnahme sind in Abbildung 1 veranschaulicht. Für die Telefongespräche wurde auf der Basis einer Onlinerecherche nach national und international bestehenden Angeboten ein Leitfaden mit folgenden Themenschwerpunkten entwickelt:

- Entstehung/Entwicklung des Angebots (u.a. Initiator, Entwicklungsdauer, Beginn)

- Allgemeines zum Angebot (u.a. Dauer, Anzahl der Teilnehmer pro Durchgang)

- Angebotsbeschreibung (u.a. Format, Teilnahmebedingungen, Leistungsnachweise)

- Organisation und Finanzierung

- Evaluation des Angebots

\section{Datenauswertung}

Die Gespräche wurden digital aufgezeichnet und stichpunktartig protokolliert. Die Antworten wurden anhand der vorgefertigten und für die Gespräche genutzten Systematik in eine Exceltabelle übertragen und entsprechend strukturiert. Tabelle 1 ist das Er-

\footnotetext{
* Die personenbezogenen Bezeichnungen werden aus Gründen der besseren Lesbarkeit in maskuliner bzw. neutraler Form wiedergegeben.

Sie beziehen sich jedoch, wenn nicht anders vermerkt, sowohl auf Frauen als auch auf Männer.

** Abzüglich des Instituts für Allgemeinmedizin der Johann Wolfgang Goethe-Universität, Frankfurt.
} 


\begin{tabular}{|c|c|c|c|c|c|c|c|c|}
\hline & 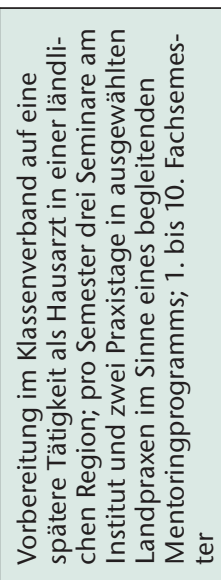 & 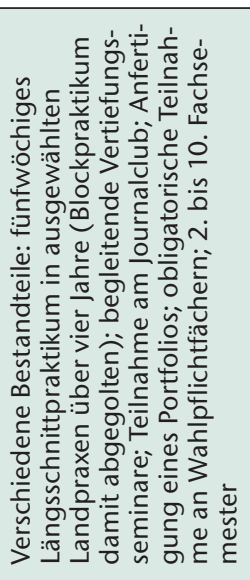 & 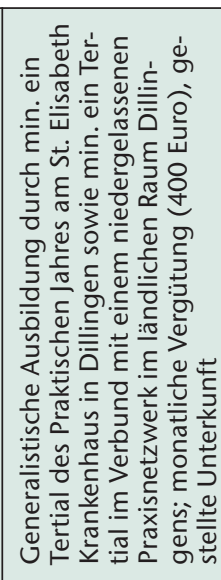 & & 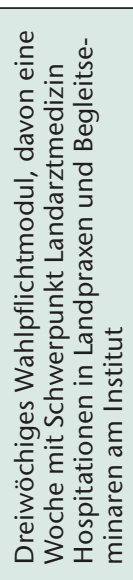 & 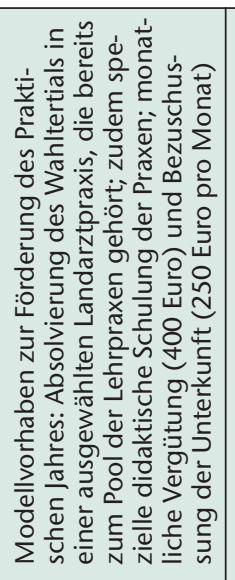 & 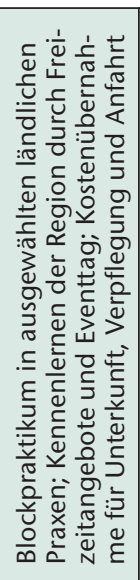 & 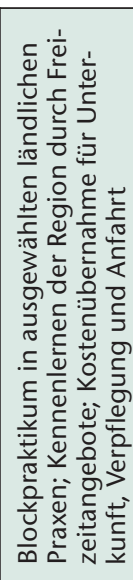 \\
\hline & 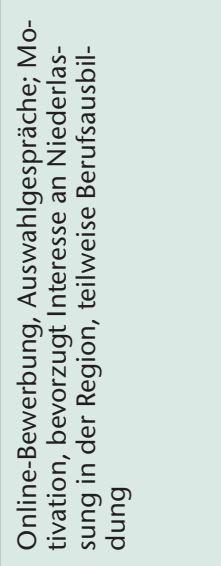 & 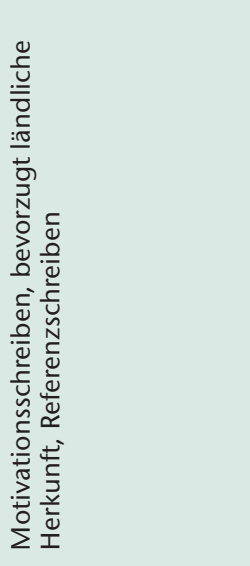 & 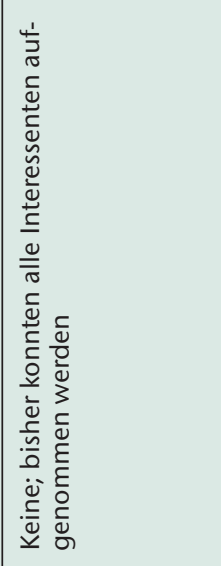 & & 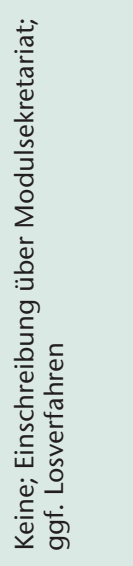 & 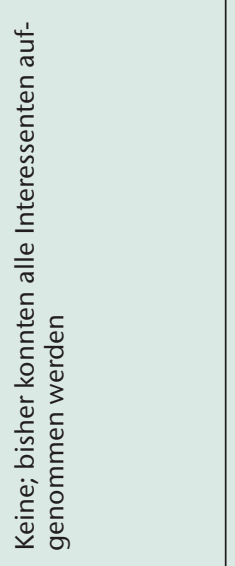 & 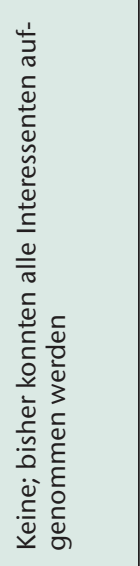 & 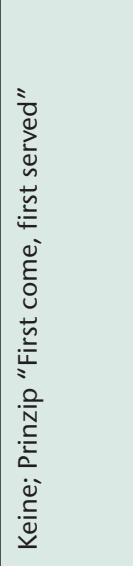 \\
\hline & $\bar{i}$ & $\stackrel{m}{\grave{N}}$ & $\stackrel{m}{\grave{N}}$ & & $\stackrel{+}{\grave{N}}$ & $\begin{array}{l}0 \\
\stackrel{0}{2} \\
i \\
\\
\end{array}$ & ָ̃ & $\stackrel{m}{i}$ \\
\hline 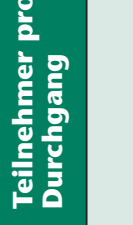 & 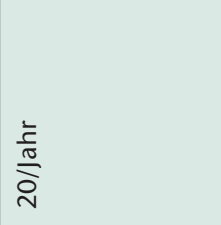 & 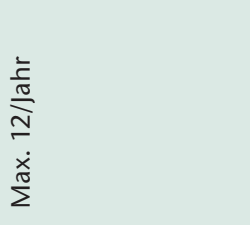 & 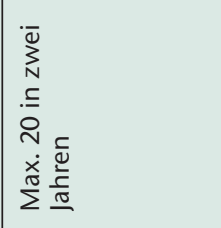 & & $\begin{array}{l}\bar{N} \\
\dot{x} \\
\stackrel{\tilde{\sigma}}{\Sigma}\end{array}$ & 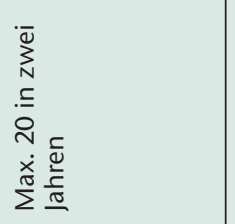 & $\begin{array}{l}\stackrel{+}{m} \\
\dot{x} \\
\stackrel{\varpi}{\Sigma} \\
\stackrel{\pi}{\Sigma}\end{array}$ & $\begin{array}{l}\circ \\
\dot{x} \\
\stackrel{x}{\Sigma}\end{array}$ \\
\hline & 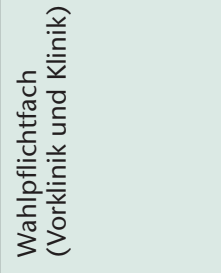 & 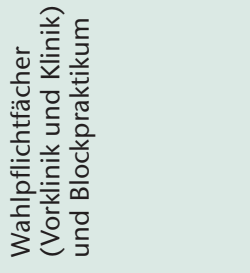 & 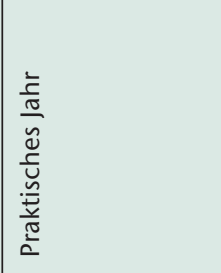 & & 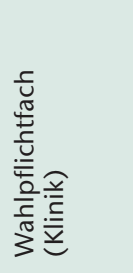 & 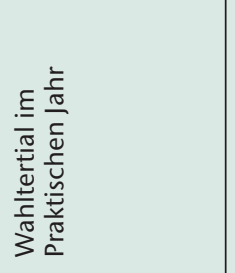 & 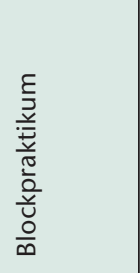 & 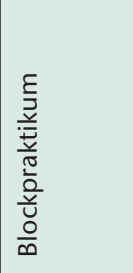 \\
\hline 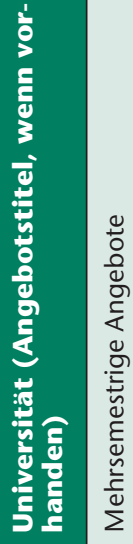 & 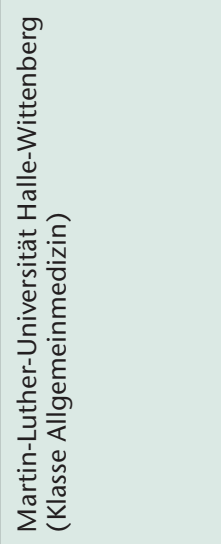 & 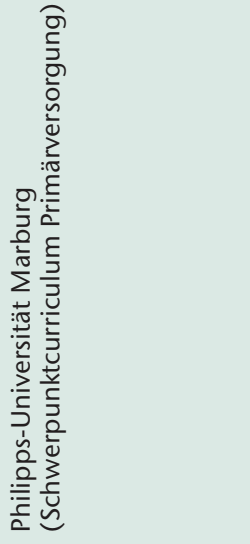 & 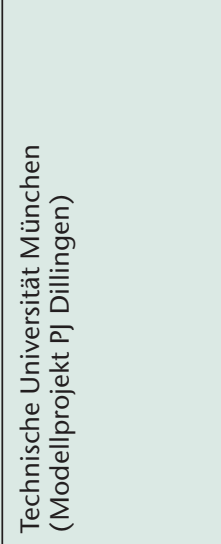 & 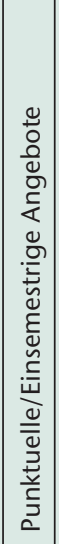 & 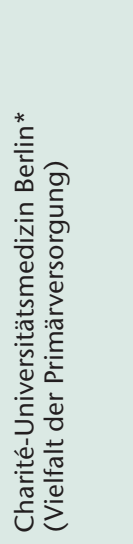 & 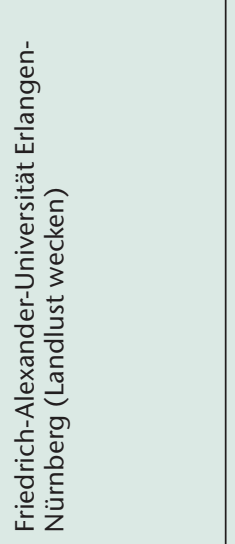 & 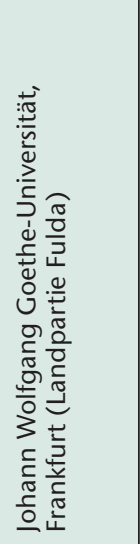 & 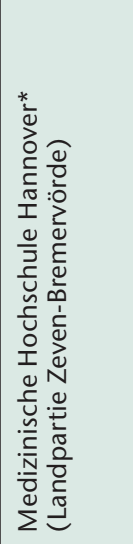 \\
\hline
\end{tabular}




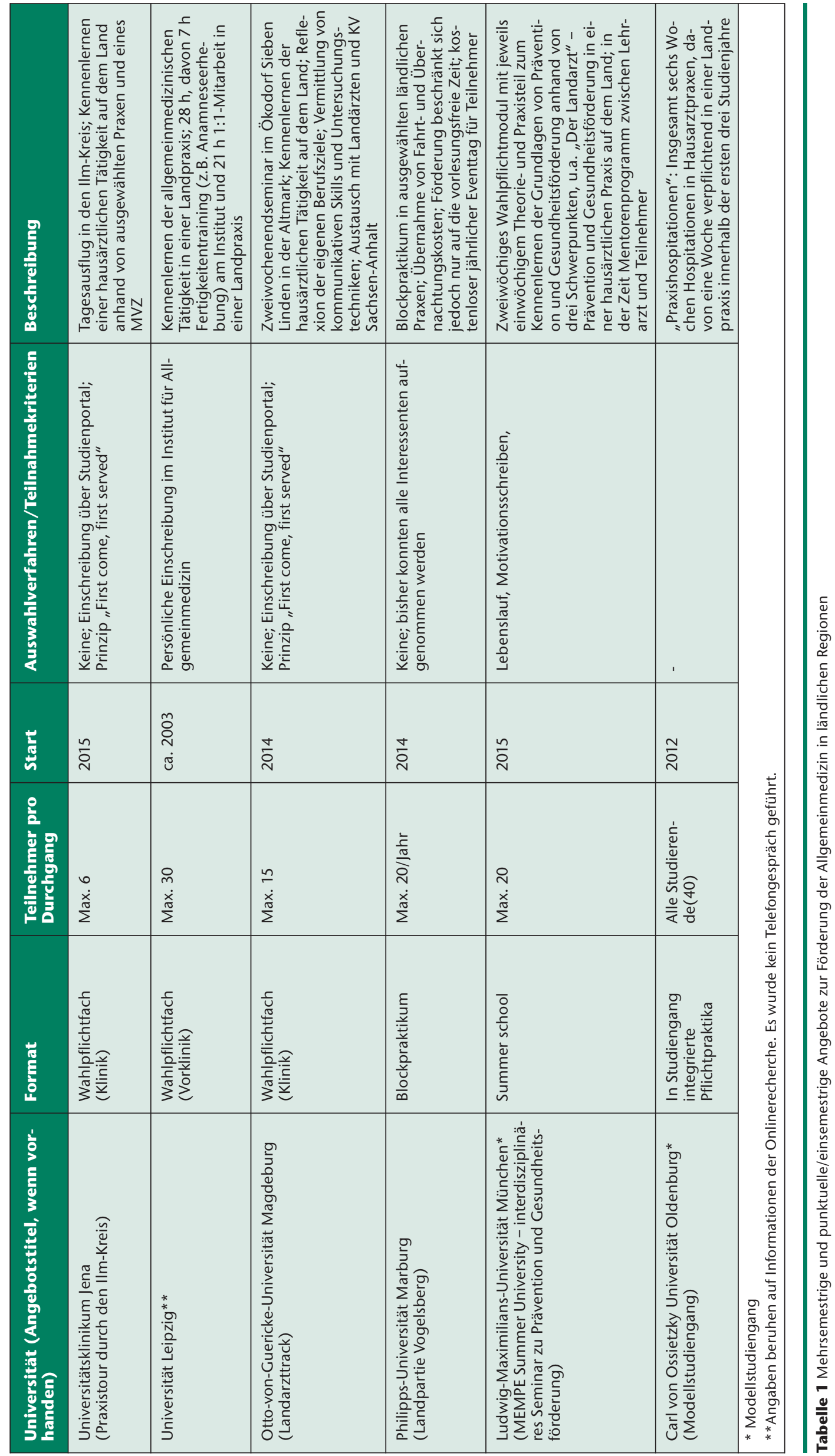


gebnis aus der Internetrecherche und den Telefongesprächen.

\section{Ergebnisse}

Die Telefongespräche dauerten zwischen 10 und 35 Minuten und fanden zwischen Ende Juli und Anfang November 2015 statt. Von insgesamt 31 der 37 universitären allgemeinmedizinischen Einrichtungen erhielten die Autoren eine Rückmeldung (83,8 \%) Zusätzlich konnte über die Onlinerecherche ein weiteres Angebot an der Universität Leipzig identifiziert werden (Abb. 1). Insgesamt existieren an 12 Universitäten jeweils ein relevantes Angebot und an einer Universität sogar zwei Angebote. An vier weiteren Universitäten (Heidelberg, Lübeck, Münster und Tübingen) sind nach eigenen Angaben Angebote in der Entwicklungsphase bzw. es liegen diesbezüglich erste Ideen vor. An der Universität Frankfurt befindet sich ein zweites Angebot in der Entwicklung. 16 Universitäten hatten kein Angebot, das die Einschlusskriterien erfüllt, und zu fünf konnte kein Kontakt hergestellt werden.

Die insgesamt 13 recherchierten Angebote wurden zunächst in mehrsemestrige gegenüber punktuellen/einsemestrigen Angeboten unterteilt. Hierbei zeigte sich, dass lediglich an drei Universitäten mehrsemestrige Angebote stattfinden (Tab. 1). Die Mehrheit der Angebote ist dabei so gestaltet, dass diese in das Curriculum integriert sind, sodass für die teilnehmenden Studierenden möglichst kein extracurricularer Zeitaufwand entsteht.

Die deutliche Mehrheit der Konzepte richtet sich an Studierende ab dem klinischen Studienabschnitt. Ausnahmen bilden hier die Universitäten in Halle und Marburg mit ihrem mehrsemestrigen Ansatz ab der Vorklinik bzw. ab dem ersten Fachsemester sowie die Universität Leipzig, deren Angebot sich ausschließlich an Studierende der Vorklinik richtet.

Mit Ausnahme der Universität Oldenburg - hier gehen alle Studierenden eines Jahrgangs für eine Woche in eine Landpraxis - ist allen Angeboten gemeinsam, dass sie sich an eine kleine Studierendenzahl von maximal 34 Teilnehmern pro Jahr/Durchgang wenden. Darüber hinaus existiert die
Mehrheit der Angebote seit maximal fünf Jahren.

Die Teilnahmekriterien für interessierte Studierende variieren stark zwischen den einzelnen Universitäten. Für acht Programme müssen die Bewerber keine speziellen Voraussetzungen mitbringen. Zumeist können alle Interessenten aufgenommen werden, da die Nachfrage das Angebot nicht übersteigt. Sollte dies jedoch der Fall sein, greift das Prinzip „First come, first served“ oder ein Losverfahren. An drei Universitäten müssen interessierte Studierende hingegen ein Motivationsschreiben und einen Lebenslauf einreichen. Für die „Klasse Allgemeinmedizin“ (Universität Halle-Wittenberg) existiert zudem ein mehrstufiges Auswahlverfahren. Ausgewählt werden dabei verstärkt Studierende, die eine erhöhte Motivation haben, später als Hausarzt im ländlichen Raum tätig zu werden und/oder die selbst aus einer ländlichen Region stammen.

Als Hauptinitiator und Organisator kann die jeweilige allgemeinmedizinische Einrichtung bzw. können einzelne Mitarbeiter/Arbeitsgruppen aus der dortigen Lehre und Forschung gesehen werden. In Einzelfällen gab es eine unterstützende Entwicklung durch weitere Akteure wie Landkreise, Ministerien oder Verbände.

Nahezu ausnahmslos konnten die Angebote zumindest auf eine Anschubfinanzierung zurückgreifen. Zum Teil existieren mehrjährige finanzielle Förderungen vorrangig für die Erstattung von Fahrt- und Übernachtungskosten. Aber auch Sach- und Personalkosten werden in einzelnen Fällen übernommen. Finanzgeber sind neben Kommunen und Landkreisen auch Ministerien, die jeweilige Kassenärztliche Vereinigung (KV), die Deutsche Apothekerund Ärztebank sowie Hausärzteverbände.

Die deutliche Mehrheit der Gesprächspartner hat angegeben, die Angebote zu evaluieren. Gleichwohl liegen nur in Ausnahmefällen entsprechende Veröffentlichungen vor [19-21].

\section{Diskussion}

An rund einem Drittel (12) der medizinischen Fakultäten konnte mindestens ein Angebot identifiziert werden, das
Studierenden Praxiserfahrungen in der Allgemeinmedizin auf dem Land ermöglicht. Davon sind drei longitudinal, d.h. auf mindestens zwei Semester angelegt. Fünf weitere Programme sind in Planung. Über deren Ausgestaltung lagen zum Zeitpunkt der Befragung noch keine detaillierten Informationen vor. Mit Ausnahme der Universität Oldenburg richten sich die Angebote nur an eine geringe Anzahl Studierender pro Durchgang und/oder Jahr.

Nahezu alle Angebote können auf eine finanzielle Unterstützung durch verschiedene Akteure zurückgreifen. Allerdings scheinen die Angebote insgesamt unterfinanziert, da kaum personelle Ressourcen für (die Veröffentlichung von) fundierte(n) Evaluationsstudien und entsprechende inhaltliche sowie organisatorische Weiterentwicklungen bestehen. Ein Programm musste als Konsequenz einer auslaufenden Finanzierung komplett eingestellt werden (Universität Ulm). Mehrjährige Finanzierungen könnten den Einrichtungen Planungssicherheit geben und dazu beitragen, dass Angebote nicht nur evaluiert und organisatorisch begleitet werden könnten, sondern zuvor bereits eine strukturierte Konzeptions- und Implementierungsphase möglich ist.

Dennoch ist die Zahl der Angebote in den vergangenen Jahren stetig gestiegen. In den Gesprächen zeigten die Rückmeldungen der Befragten, dass die Stärkung der hausärztlichen Versorgung auf dem Land während des Studiums ein Thema von zunehmender Bedeutung für die akademischen Lehreinrichtungen ist. Die Gesprächspartner wiesen ein hohes Problembewusstsein für die hausärztliche Versorgung in ländlichen Regionen auf. Sie äußerten zum Teil den Wunsch, sich über diese Problematik mit anderen betroffenen Universitäten, der Politik und relevanten Akteuren des Gesundheitssystems auszutauschen.

Ein Vergleich mit dem angloamerikanischen Raum zeigt, dass hiesige Ansätze nicht an die Intensität und Qualität der dortigen, zum Teil seit Jahrzehnten existierenden Angebote heranreichen. Exemplarisch sei hier das „Physician Shortage Area Program“ (PSAP) am Jefferson Medical College in Philadelphia, USA genannt. Das 1974 initiierte Programm richtet sich an Studierende mit einer ländlichen oder kleinstädti- 


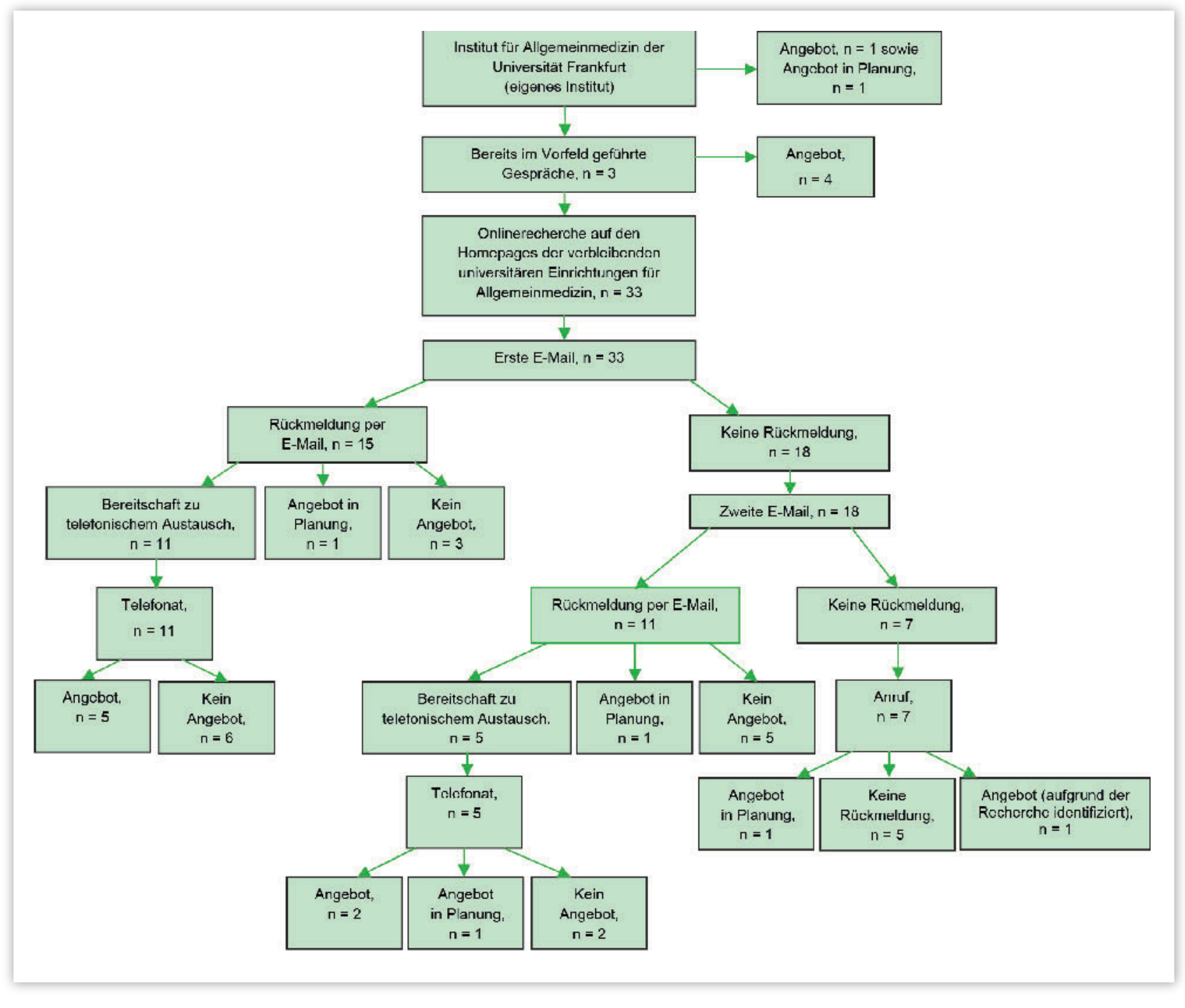

Abbildung 1 Flow Chart - Rekrutierung und Datenerhebung (eigene Darstellung)

schen Herkunft und einem grundlegenden Interesse an der Allgemeinmedizin. PSAP-Teilnehmer absolvieren u.a. ihr mindestens sechswöchiges „clinical clerkship“ an einem „Landkrankenhaus", verbringen ihr vierwöchiges „subinternship“ möglichst in einer ländlich gelegenen Allgemeinmedizinpraxis und nehmen an einem Mentoringprogramm teil [23]. Evaluationsergebnisse konnten zeigen, dass PSAPAbsolventen im Vergleich zu Studienkollegen, die nicht am Programm teilgenommen haben, auch noch mindestens fünf Jahre nach ihrem Abschluss sowohl häufiger als Allgemeinmediziner $(32,0 \%$ vs. $3,2 \%$; $R R=9,9)$, als auch in ländlichen Gebieten Pennsylvanias tätig sind $(24,7 \%$ vs. $2,0 \%$; RR $=12,4)$ [24]. Weitere Ansätze und Programme mit ähnlichem Format existieren weltweit $[10-15,25,26]$.
Gleichzeitig bedeutet dies nicht, dass diese Ansätze eins zu eins auf Deutschland übertragen werden sollen bzw. eine uneingeschränkte Adaption überhaupt möglich wäre. Grundlegende Unterschiede in Studiengangstrukturen, Finanzierungsquellen und der Definition von „ländlicher Raum“ führen sicherlich $\mathrm{zu}$ verschiedenen Voraussetzungen und Bedarfen für die Entwicklung und Umsetzung von Landarztprogrammen in den einzelnen Ländern.

Trotz der unterschiedlichen und hierzulande zum Teil begrenzenden Rahmenbedingungen können etablierte Programme aus dem Ausland als Orientierung und Ideengeber dienen. Sie können dazu beitragen, dass in Deutschland in Zukunft mehr kreative, praxisnahe, neigungsorientierte und longitudinale Ansätze auch im Rahmen von Regelstudiengängen geschaffen werden.
Im Gegensatz zu den genannten ausländischen Programmen liegen in Deutschland aufgrund der zu kurzen Laufzeiten der Programme (noch) keine Evaluationsergebnisse dazu vor, ob die Teilnahme am Angebot die Wahrscheinlichkeit erhöht, später als niedergelassener Hausarzt auf dem Land zu arbeiten. Aktuell konnten erste Untersuchungsergebnisse der Universitäten Frankfurt [19], Magdeburg [20] und Leipzig [21] jedoch belegen, dass zumindest das Interesse an der Allgemeinmedizin und daran, später als niedergelassener Hausarzt zu arbeiten, durch die Teilnahme an den Angeboten steigt. Diese Ergebnisse decken sich mit der Literatur, die positive Effekte auf die spätere Berufswahl - hier auf die Entscheidung, als niedergelassener Hausarzt im ländlichen Raum zu arbeiten - durch longitudinale Lehrkonzepte beschreibt $[26,27]$. 


\section{Linda Barthen, M.Sc. Public Health ...}

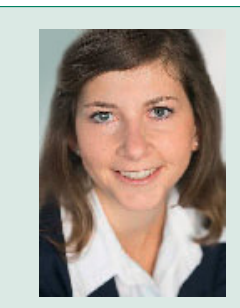

... ist Gesundheitswissenschaftlerin und seit 2014 wissenschaftliche Mitarbeiterin am Institut für Allgemeinmedizin der Goethe-Universität, Frankfurt am Main. In ihren Projekten befasst sie sich neben der Sicherstellung der hausärztlichen Versorgung in ländlichen Regionen mit der konzeptionellen Entwicklung sowie Umsetzung von allgemeinmedizinischen Schwerpunktprogrammen im Medizinstudium.
Trotz fehlender Langzeitstudien wären aber auch Evaluationsergebnisse zu Erfolgsfaktoren der jeweiligen Programme wünschenswert. Warum nehmen die Studierenden an den freiwilligen Landarztprogrammen teil? Sind beispielsweise die Zusatzangebote wie Eventtage, die extra geschulten Lehrpraxen, die Begleitseminare oder am Ende doch die finanziellen Anreize ursächlich? Weitere Fragestellungen zur Prozess- und Ergebnisevaluation sind hier denkbar.

Insgesamt gibt es für die Initiierung eines Angebots zwei wesentliche, wenn zuweilen auch triviale Voraussetzungen - einen „Kümmerer“ vor Ort, der die Entwicklung und Umsetzung des jeweiligen Angebots vorantreibt sowie mindestens eine Anschubfinanzierung. Sowohl an der einen wie auch an der anderen Voraussetzung scheint es aktuell mancherorts zu scheitern.

\section{Stärken und Schwächen der Arbeit}

Die vorliegende Untersuchung ist die erste umfassende Übersicht zu aktuellen Förderangeboten der Allgemeinmedizin im ländlichen Raum während des Studiums. Allerdings erhebt die Zusammenstellung keinen Anspruch auf Vollständigkeit, da der Rücklauf bei 83,8 \% lag. Es ist durchaus möglich, dass weitere, hier nicht gelistete Angebote existieren.

Ein Vergleich zwischen den Universitäten sollte mit Vorsicht erfolgen und ist nur eingeschränkt möglich. Es ist anzunehmen, dass sich die Möglichkeiten und Grenzen der Gestaltung sowie der Umsetzung von Angeboten unterscheiden in Abhängigkeit davon, ob es sich um Regelstudiengänge, Modellstudiengänge oder auch Schwerpunktcurricula handelt. Eine Bewertung der einzelnen Angebote wurde daher in der vorliegenden Arbeit bewusst nicht vorgenommen.

\section{Schlussfolgerungen}

Die Ergebnisse der Untersuchung zeigen, dass Angebote, die die Allgemeinmedizin in ländlichen Regionen während des Studiums stärken sollen, eine zunehmende Bedeutung an deutschen medizinischen Fakultäten erhalten. Es handelt sich jedoch um ein noch sehr junges Themenfeld, sodass im Vergleich zu internationalen Flächenstaaten mit einer entsprechend längeren Historie deutlich wird, dass hiesige Programme noch in den Kinderschuhen stecken. Für die Zukunft sollten begleitende Evaluationen zeigen, welche der gewählten Ansätze einen positiven Beitrag zur Gewinnung allgemeinmedizinischen Nachwuchses für ländliche Regionen leisten können. Kooperationen zwischen den Universitäten und ein gegenseitiges Lernen voneinander könnten möglicherweise die Qualität der Angebote steigern.

Interessenkonflikte: keine angegeben.

\section{Korrespondenzadresse}

Linda Barthen

Goethe-Universität Frankfurt am Main

Theodor-Stern-Kai 7

60590 Frankfurt am Main

Tel.: 069 6301-4926

Barthen@allgemeinmedizin.uni-frankfurt.de

\section{Literatur}

1. Korzilius H. Hausärztemangel in Deutschland: Die große Landflucht. Dtsch Arztebl 2008; 105: A-373 / B-335 / C-331

2. Roos M, Hartleb L, Langbein SH. Der Hausarztmangel auf dem Land ist angekommen. Z Allg Med 2015; 91: 131-136

3. Bundesärztekammer. Ärztestatistik 2015 www.bundesaerztekammer.de/filead min/user_upload/downloads/pdfOrdner/Statistik2015/Stat15Abb-

Tab.pdf (letzter Zugriff am 31.05.2016)

4. Bundesärztekammer. Ärztestatistik 2013. www.bundesaerztekammer.de/filead min/user_upload/downloads/Stat13 AbbTab.pdf (letzter Zugriff am 31.03.2016)

5. Heinz A, Jacob R. Medizinstudenten und ihre Berufsperspektiven. In welcher Facharztrichtung, wo und wie wollen sie arbeiten? Bundesgesundheitsbl 2012; 55: 245-253

6. MacKean P, Gutkin C. Fewer medical students selecting family medicine. Can Fam Physician 2003; 49: 408-409
7. Bethune C, Hansen PA, Deacon D, Hurley K, Kirby A, Godwin M. Family medicine as a career option. How students' attitudes changed during medical school. Can Fam Physician 2007; 53: 880-885

8. Federal Office of Rural Health Policy. Facts about ...rural physicians. www. shepscenter.unc.edu/rural/pubs/finding_brief/phy.html (letzter Zugriff am 23.05.2016)

9. Joyce C, Wolfe R. Geographic distribution of the Australian primary health workforce in 1996 and 2001. Aust N Z J Public Health 2005; 29: 129-135

10. Inoue K, Matsumoto M, Sawada T. Evaluation of a medical school for rural doctors. J Rural Health 2007; 23: 183-187

11. Putthasri W, Suphanchaimat R, Topothai T, Wisaijohn T, Thammatacharee N, Tangcharoensathien V. Thailand special recruitment track of medical students. Hum Resour Health 2013; 47
12. Krupa LK, Chan BT. Canadian rural family medicine training programs: growth and variation in recruitment. Can Fam Physician 2005; 51: 852-853

13. Rabinowitz HK, Diamond JJ, Markham FW, Wortman JR. Medical school programs to increase the rural physician supply: a systematic review and projected impact of widespread replication. Acad Med 2008; 83: 235-243

14. Strasser RP, Lanphear JH, McCready WG, Topps MH, Hunt DD, Matte MC. Canada's new medical school. Acad Med 2009; 84: 1459-1464

15. The University of Western Australia. The Rural Clinical School of WA. www. rcs.uwa.edu.au/ (letzter Zugriff am 29.04.2016)

16. Farmer J, Kenny A, McKinstry C, Huysmans RD. A scoping review of the association between rural medical education and rural practice location. Hum Resour Health 2015; 13: 27

17. MacDowell M, Glasser M, Hunsaker M. A decade of rural physician workforce 
outcomes for the Rockford Rural Medical Education (RMED) Program, University of Illinois. Acad Med 2013; 88: 1941-1947

18. Steger T, Langosch C, Klement A, Onnasch J. „Klasse Allgemeinmedizin“. Z Allg Med 2012; 88: 264-267

19. Schäfer H, Mangold B, Paulitsch M, Gerlach FM. Landpartie Fulda - ein Projekt zur Förderung des hausärztlichen Nachwuchses im ländlichen Raum. Z Allg Med 2015; 91: 260-263

20. Herrmann M, Hänel P, Jansen E. Landärztliche Ausbildung zur Reduzierung der medizinischen Unterversorgung auf dem Land-Erfahrungen, Chancen, Widrigkeiten. In: Brunnett $\mathrm{R}$ et al. (Hrsg.). Jahrbuch für Kritische Medizin und Gesundheitswissenschaften 50. Hamburg: Argument Verlag, 2014: 144-172
21. Deutsch T, Hönigschmid P, Frese T, Sandholzer H. Early community-based family practice elective positively influences medical students' career considerations - a Pre-post-comparison. BMC Fam Pract 2013; 14: 24

22. Fricke A. Neue Debatte um Landarztquote. www.aerztezeitung.de/politik gesellschaft/bedarfsplanung/default. aspx?sid=903084\&cm_mmc=News letter-_-Newsletter-C-_-20160120-_-Bedarfsplanung (letzter Zugriff am 23.08.2016)

23. Jefferson Medical College. Physician Shortage Area Program. www.jefferson. edu/university/skmc/programs/physician-shortage-area-program.html (letzter Zugriff am 05.07.2016)

24. Rabinowitz HK, Diamond JJ, Markham FW, Santana AJ. Increasing the supply of rural family physicians: recent outcomes from Jefferson Medical College's Physician Shortage Area Program (PSAP). Acad Med 2011; 86: 264-269

25. Verma P, Ford JA, Stuart A, Howe A, Everington S, Steel N. A systematic review of strategies to recruit and retain primary care doctors. BMC Health Serv Res 2016; 16: 194

26. Pfarrwaller E, Sommer J, Chung C, et al. Impact of interventions to increase the proportion of medical students choosing a primary care career. J Gen Intern Mer 2015; 30: 1349-1358

27. Deutsch T, Lippmann S, Frese T, Sandholzer H. Gewinnung hausärztlichen Nachwuchses - Zusammenhang zwischen praxisorientierter Lehre und Karriereentscheidung. Gesundheitswesen 2014; 76: 26-31 


\title{
How can General Practice be incorporated longitudinally in medical studies? Students' views on the development of a new rural health program
}

\begin{abstract}
Aim: Participation of medical students in the conceptual development of targeted and attractive teaching content for rural areas.

Method: A questionnaire was developed to gather information on students' views of their current medical studies, career interests, and what requirements should be met by an optional rural health program in general practice. By means of an online survey in summer 2015, all medical students from the fourth preclinical semester onwards $(n=2,150)$ at Goethe University Frankfurt were surveyed on one occasion. Statistical analysis was mainly descriptive. Personal attitudes towards a career as a family practitioner were examined for statistical significance. Further information was gathered on whether a measurable correlation exists between personal background and desired work location.

Results: Of the 2,150 students that were contacted, 617 participated in the survey (response rate $=28.7 \%$ ). The results covered a wide range of ideas and recommendations and were representative both of medical students with a positive attitude toward general practice, as well as those that were rather critical of teaching in general practice. The students expected the planned health program to be of strong practical relevance and to acquaint them with the administrative and economic aspects of running a practice.

Conclusions: By including the target group in the development process, it was possible to tailor the health program to meet the needs of future participants more precisely. Student participation can also be expected to result in greater acceptance of the program. The results on teaching content may also provide other medical faculties with orientation when developing comparable programs.
\end{abstract}

Keywords: general practice, medical students, curriculum, rural health program, shortage of family doctors

\section{Introduction}

Against the background of an increasing shortage of GPs, it is essential to attract a new generation of family doctors, particularly to the rural areas of Germany. The use of designated health programs during medical studies to promote general practice can have a positive effect on readiness to work as a family doctor (in rural areas) after completion of studies [1]. In foreign countries, and particularly in the U.S., Canada and Australia, numerous health programs already exist that are aimed at promoting general practice during medical studies [2], [3]. The number of such programs is also increasing in Germany [4], [5]. There are signs that the acquisition of skills during special general practice programs has a positive influence on the likelihood that a student will choose to specialize in general practice [6], and thus promotes general practice as a preferred career option [7].

\author{
Linda Barthen ${ }^{1}$ \\ Gisela Ravens-Taeuber ${ }^{1}$ \\ Michael A. Paulitsch ${ }^{1}$ \\ Ferdinand M. Gerlach ${ }^{1}$ \\ Monika Sennekamp ${ }^{1}$
}

1 Goethe University Frankfurt, Institute of General Practice, Frankfurt/Main, Germany 
volved when curricular changes and innovations of this kind are planned [11]. As experts in matters affecting them personally, students know where the deficits and potential improvements in the current curriculum are, and are very interested in being involved in the change process [12].

The aim of the survey presented here was to include students in the conceptual development of a new "rural health program" (also to be referred to as "priority program"). The focus lay on the question what the students wanted and expected of a priority program aimed at promoting general practice in rural areas. Furthermore, information was collected on the students' desired careers and their evaluation of general practice as part of their medical studies.

\section{Methods}

\section{Data collection}

An invitation to participate in a web-based survey with a cross-sectional design was sent to all students in their fourth preclinical semester or above and studying at Goethe University Frankfurt. They were contacted via the e-mail distribution lists of the Dean's Office and the Institute of General Practice. The choice of student population was based on the assumption that from the fourth preclinical semester onwards students had sufficient knowledge of the way their course was structured and what awaited them in the future. Furthermore, e-mail distribution lists existed for these students, so direct contact was possible. The e-mail included a short information letter and the link to the online-questionnaire. At the end of May, the entire population of 2,150 students was contacted for the first time. A reminder was sent mid-June 2015.

\section{Survey instrument}

The specially designed questionnaire (see attachment 1) was based on the results of a nationwide survey of medical students that took place in the year 2015 [13] and was further developed and agreed upon by an interdisciplinary team consisting of a physician in specialist training, an experienced GP and specialists in educational science, psychology and the health sciences.

The questionnaire was tested on three students and subsequently adapted. The final version of the questionnaire included 19 items broken down into four thematic sections - socio-demographic information, career choice, medical studies, and comments on the rural health program to be developed. They were also asked to gauge their inclination to set up as a GP in private practice on a six-point scale (1=“True"; 6="False"). In addition to mostly closed questions, the questionnaire included five open questions.

In the final thematic section, the participants were first presented with an initial idea of what the future priority program might look like, based on the literature and other established programs (components: training in a practice, accompanying seminar, and mentoring program [z.B. [14], [15], [16], [17]. Participants were subsequently required to comment on the outlined idea and then to say whether they would take part in such a program.

The Survey Monkey tool was used to ensure low-threshold access. In this way, it was hoped that students that had shown little or no interest in general practice to date, or were critical of the discipline, could also be encouraged to participate.

The ideas for the rural health program that stemmed from the survey were agreed upon in a subsequent discussion session with individual faculty students.

\section{Data analysis}

Data were mostly descriptively analyzed using the IBM SPSS statistics software. Absolute and relative frequencies were calculated for categorical variables. Possible differences in average values for readiness to work as a GP were tested for statistical significance using a paired Wilcoxon test. The effect size of any differences were quantified by calculating Cohen's d: 0.2-0.4=small effect; $0.5-0.8=$ moderate effect and $>0.8=$ large effect [18]. In addition, any correlation between background and desired place of work was tested using Spearman's correlation coefficient ( $\alpha \leq 5 \%)$.

Socio-demographic information was presented by reducing the six-levels of the rating scale on personal background to a dichotomous rural community or town answer [19].

Free-text answers were analyzed using quantitative content analysis, with relevant attributes of text passages being identified and operationalized via allocation to a developed system of categories. Frequencies of allocation to individual categories were regarded as indicators of text attributes [20]. Participants in the survey generally answered in whole sentences, or by naming key points. Single points were regarded as single units (e.g. question 13 a "Receive own patients for a preliminary investigation"), longer presentations of key points or several sentences were divided into several units, depending on content (e.g. question 13 a "Practice should make at least one investigation room available to students, and it would also be good to be able to perform instrumentbased diagnostics". The individual units were then allocated to the categories (e.g. "Receive own patients for a preliminary investigation"=Do practical work; "Practice should make at least one investigation room available to students"=Own treatment room; “... instrument-based diagnostics..." Get to know and use instrumental diagnostic procedures) that were previously derived from the text material (inductive approach). Two persons were used to analyze and encode the open texts. Only those categories that were named at least 10 times were presented in the results (see table 1 ). 
Table 1: Information on the three elements of the rural health program. (Evaluation of open questions; Illustration of categories mentioned at least 10 times).

\begin{tabular}{|c|c|c|c|}
\hline \multicolumn{4}{|c|}{ PRACTICAL TRAINING/INTERNSHIP AT A FAMILY PRACTICE, N = 329} \\
\hline & & n & $\%$ \\
\hline$\bullet$ & Practical work & 207 & 62.9 \\
\hline$\bullet$ & Regular and detailed feedback provided by family doctor/mentor & 115 & 35.0 \\
\hline \multicolumn{4}{|c|}{ - Organization and circumstances } \\
\hline & Own treatment room & 22 & 6.7 \\
\hline & 1:1 support & 19 & 5.8 \\
\hline & Highly motivated and dedicated family doctors/support & 15 & 4.6 \\
\hline & Free choice of family practice & 12 & 3.7 \\
\hline & - Organized arrival and departure (to/from the practice) & 12 & 3.7 \\
\hline$\bullet$ & Get to know and use diagnostic equipment (e.g. ECG, Sonography) & 29 & 8.8 \\
\hline & $\begin{array}{l}\text { Get to know additional work of a family doctor (home visits, visits to care homes, provide } \\
\text { support to HCAs, emergency services) }\end{array}$ & 27 & 8.2 \\
\hline • & Practice management (computer system, invoicing, coding, documentation etc.) & 21 & 6.4 \\
\hline \multicolumn{4}{|c|}{ SEMINARS, $N=303$} \\
\hline & \multicolumn{3}{|l|}{ Topics for the seminars } \\
\hline & $\begin{array}{l}\text { Practical tutorials - from anamnesis to therapy based on typical disease patterns in } \\
\text { family medicine, }\end{array}$ & 129 & 42.6 \\
\hline & - Sharing experiences, case reviews after practical training/internship & 88 & 29.0 \\
\hline & $\begin{array}{l}\text { Practice management (computer system, invoicing, coding, documentation etc.) and } \\
\text { getting to know different forms of practice organization }\end{array}$ & 30 & $\begin{array}{l}9.9 \\
9.2\end{array}$ \\
\hline & - Practical tutorials - diagnostic equipment (e.g. ECG, Sonography, wound care) & 28 & \\
\hline & $\begin{array}{l}\text { Soft skills/social competencies/communication training/dealing with ("complicated") } \\
\text { patients and situations }\end{array}$ & 21 & 6.9 \\
\hline & Emergency care; Referral to other medical doctors & & \\
\hline & Comparison urban/rural healthcare; advantages and disadvantages of working as a & & 3.7 \\
\hline & family doctor in a rural area & $\begin{array}{l}12 \\
12\end{array}$ & 3.7 \\
\hline \multicolumn{4}{|c|}{ - Organization und circumstances } \\
\hline & No/few student presentations & 27 & 8.9 \\
\hline & Interactive, no/little teacher-centered learning, workshops, topics chosen by students & 20 & 6.6 \\
\hline & Very small groups & & \\
\hline & Real patients/real medical cases/patient actors & $\begin{array}{l}20 \\
20\end{array}$ & $\begin{array}{l}6.6 \\
6.6\end{array}$ \\
\hline \multicolumn{4}{|c|}{ MENTORING PROGRAM, N = 259} \\
\hline \multicolumn{4}{|c|}{ - Family doctor as mentor } \\
\hline & Trustworthy and prepared to answer questions of any kind & 65 & 25.1 \\
\hline & Hints/targeted assistance/feedback during internship & 61 & 23.6 \\
\hline & Advice on occupational and personal development & 21 & 8.1 \\
\hline & - Joint case reviews/discussion of therapy strategies & 18 & 6.9 \\
\hline & $\begin{array}{l}\text { Development of practical skills (gain and make use of practical experience, work } \\
\text { independently, gain confidence dealing with patients) }\end{array}$ & 70 & 27.0 \\
\hline \multicolumn{4}{|c|}{ - Organization and circumstances } \\
\hline & - Good 1:1 support/small groups & 32 & 12.4 \\
\hline & Easy access to family doctor & 30 & 11.6 \\
\hline & - Highly motivated mentors that find enough time to teach support & 28 & 10.8 \\
\hline & $\begin{array}{l}\text { Realistic insight into practice management (computer system, invoicing, coding, } \\
\text { documentation etc.) }\end{array}$ & 38 & 14.7 \\
\hline & Benefit from the family doctors' experience & 27 & 10.4 \\
\hline
\end{tabular}

\section{Results}

After cleansing the data, 617 questionnaires were suitable for analysis (response rate=28.7\%). 423 participants were female (68.6\%). The median year of birth was 1990 (range: 1968-1995), and on average the participants were in their $8^{\text {th }}$ semester (range: $2^{\text {nd }}-16^{\text {th }}$ semester; one person said they were in their $2^{\text {nd }}$ semester). Most participants grew up in a town ( $>5,000$ inhabitants). Around one fifth $(21.0 \%)$ came from a rural community $(<5,000$ inhabitants). Furthermore, nearly one fifth (18.5\%) said they had completed vocational training in another profession. Only $4.5 \%$ had children.

\section{Current study situation and career choice}

When asked at what stage in their studies medical students should gain their first practical experience in a family practice, almost one quarter (23.5\%) said it should be in the preclinical phase. A clear majority of nearly twothirds (65.8\%) regarded the clinical stage of their studies as the right time. Only $5.4 \%$ were in favor of such practical training before beginning their studies. Differences existed between students in preclinical and clinical stages of their studies, with $41.7 \%$ of those in the preclinical phase regarding the preclinical phase as suitable, while only $17.6 \%$ of those in the clinical phase did.

The questionnaire did not collect data on students' experience of general practice, but responses could be expected to depend on the year of studies a student was in. Students that considered a career in general practice to be possible were asked whether they thought their education to date had prepared them sufficiently. The further a student had progressed in their studies, the more likely the answer was to be yes. Nonetheless, $19.0 \%$ of respondents from the $11^{\text {th }}$ semester onwards $(N=158)$ said they did not feel sufficiently prepared, and $55.1 \%$ said they were only partially prepared (74.1\% overall). The respondents said that in order to feel sufficiently prepared $(N=97$; open question), they would need more practical experience (53.6\%), to be acquainted with the administrative and economic aspects of running a practice (15.5\%), and more interdisciplinary knowledge (11.4\%). 
A basic interest in working in family medicine was also measured using a 6-point rating scale (1="True"; $6=$ "Untrue"). The participants were asked whether "at the beginning of their studies, they could imagine working as a family doctor in the future" (average 3.81) and whether they "could now imagine working as a family doctor in the future" (average 3.36). A comparison of the results using the Wilcoxon test showed that interest increased statistically significantly during the course of studies $(p<0.001 ; N=617)$. At 0.278 , Cohen's d showed a small effect size [18]. Furthermore, $43.5 \%$ of students $(n=268)$ said they could not really or not at all imagine later working as a GP. Nevertheless, $46.7 \%$ of these $(\mathrm{N}=125)$ made specific proposals and had definite ideas on how to structure practical training. These results can be seen in Table 1.

Figure 1 shows the preferred number of inhabitants living in the future place of work. A statistically significant correlation exists between the variables of own background and desired place of work with a moderate effect size $(r=0.366 ; p<0.001 ; N=458)$ [20]. Almost one third of participants from rural communities said they had no preferences with regard to future place of work.

\section{Information on the planned rural health program}

To structure the priority program, the students were first provided with a preliminary concept (part I: training in a practice; part II: accompanying seminar; part III: mentoring program). Around one half of participants took the opportunity to formulate their own requirements of a rural health program in general practice and express their own ideas in free text.

$62.9 \%$ of participants mentioned active practice involvement as the most important requirement of training in a family practice (part 1). This predominantly involved treating their own patients independently, from taking a medical history through diagnostic investigations to making a therapy recommendation (initially) under supervision. In addition, more than a third of participants (35\%) wanted regular and detailed feedback from the teaching physician. With regard to accompanying seminars in small groups at the university (part II), the students mainly mentioned specific content for inclusion in the seminars. Practical exercises (42.6\%) and discussion of experience and cases after the practice phase $(29.0 \%)$ were considered particularly important. The participants expected of mentoring (part III) that a family practitioner should provide them with theoretical and practical tips and feedback (23.6\%) and show a readiness to answer all types of question (25.1\%). The students hoped the mentoring would enable them to develop practical skills, for example by means of practical training (27.0\%). Complete results can be found in table 1 .

Students' responses on the desired length of the health program varied (answers were predefined). More than a quarter $(27.7 \%)$ were in favor of one semester, with a two further quarters either wanting it to last several semesters
(23.0\%), or not specifying a length as long as the "course is useful to me" (23.5\%). Fewer than $5 \%$ wanted a priority program that continued throughout the entire length of medical studies.

Finally, the participants were asked whether they would participate in the outlined course (yes $=45.4 \%$; no $=17.5 \%$; don't know=30.6\%; no response $=6.5 \%$ ). Those that were against participation attributed their decision to "no interest in general practice" (61.5\%), "no interest in working in a rural area" (45.0\%), "no time during studies" (41.3\%), "no interest in setting up in private practice" (21.1\%), and "no interest in further practical training" (21.1\%).

\section{Discussion}

617 medical students from the fourth preclinical semester onwards used the online questionnaire to participate in the development of a general practice priority program for rural areas.

The need for more practical training in medical studies is reflected in the results of the present study and the requirements that students thought the planned rural health program should satisfy. The potential participants saw a need for greater practical orientation in all three core areas (training in a practice, accompanying seminar, and mentoring program). An interest in treating patients, further developing personal skills and being actively involved in day-to-day practice life was mentioned in the open questions. Furthermore, it was seen as important to be familiarized with practice management and practice organization. This is therefore a field that should be considered in the priority program.

Regardless of specialization, more than half the participants $(54.0 \%)$ would prefer to work in a mid-sized or large town (>20.000 inhabitants). It is unclear whether this rules out "living in a town and working in the country" as a possible model for the future. It has often been noted that students with a rural background often show a greater readiness to return to the country [1], [21]. The fact that almost a third (31.3\%) of students with a rural background are undecided as to where they would like to work, indicates that this target group should be encouraged to participate in the priority program in order to awaken their interest in a career in a rural region early on.

Almost three-quarters of all participants (74.1\%) from the 11th semester onwards did not feel they had been prepared or adequately prepared for a future career in general practice. The reasons for this are often a lack of practical experience, but also insufficient knowledge of the administrative and economic know-how required to run a practice. The results present no reason for alarm, as specialist training in general practice is supposed to fulfil these needs. However, the aim of medical studies is "... to teach basic knowledge, skills and proficiency in all the disciplines that are necessary to provide comprehensive healthcare to the population" [https://www.gesetze-im-internet.de/_appro_2002/BJNR240500002. 


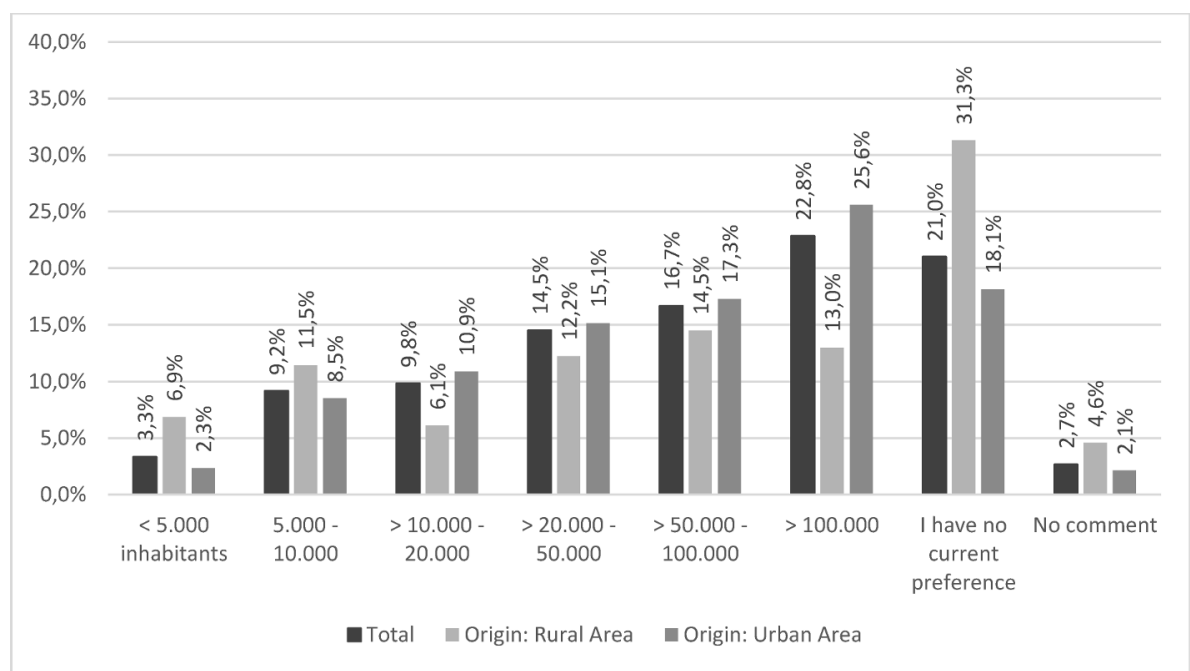

Figure 1: Preferred number of inhabitants living in future place of work according to background, N=617.

html]. Nonetheless, a survey of graduates was able to show that the lack of practical orientation and insufficient teaching of practical, medical skills are seen as weaknesses of medical studies [22]. Policymakers have also recognized these problems and are now actively trying to do something about them as part of the Masterplan Medical Studies 2020. The intention of the Masterplan is not only to raise the share of general practice in medical studies, but also to strengthen training in a family practice setting [23].

The investigation was also able to show that students' interest in possibly working in general practice increased during the course of their studies. A 2015 study carried out by Jacob, Kopp und Schultz came to a similar conclusion [13]. It should further be noted that although the rural health program could only be described in broad outlines at the time of the survey, and it is extremely specialized (general practice in rural areas), $45.4 \%$ of participants were able to imagine taking part in it. Although it cannot be assumed that actual interest will be correspondingly high, it is obvious that there is a need for such a program and that it makes sense to develop one.

The results presented here have some limitations. The questionnaire was developed specifically for the study and has not been validated. It should also be taken into consideration that cross-sectional surveys are not able to assess longitudinal changes that occur in students during the course of their studies, so divergences between cohorts in successive years may be due to factors that were not revealed here. As far as transferring the approach and the developed concept to other German study locations is concerned, it should be borne in mind that conditions differ between universities. The present results are therefore not representative for Germany as a whole and implementation elsewhere should be adapted to take account of local circumstances. On the other hand, one strength of the study is the participation of students in the development of the curriculum. It could be shown that students that were critical of general practice, or that had no interest in a career in family medicine, also parti- cipated in the survey. A broad range of views could thus be captured, making our approach preferable to the use of focus groups, in which those with no interest in general practice would not have participated. Nevertheless, in order to benefit from student feedback, we presented and agreed upon the developed concept in a discussion group with individual faculty students.

\section{Conclusions}

Overall, the participation of students helped to give concrete form to the content, organization and thematic design of the country doctor program. Initial ideas could be further developed, new approaches generated and observations that had not previously been considered taken into account. For example, the decision not to provide the priority program before the clinical stage of medical studies was made on the basis of the results. Furthermore, issues concerning running a practice and practice management will be included in the course of seminars. At heart, the program will and must demonstrate continual practical relevance, which, among other things, will be manifested in practical exercises during the seminars.

It is also to be expected that the inclusion of the target group will raise acceptance of the program. However, it may make sense in the future to consider finding ways to intensify and improve feedback from the students so their opinions continue to be reflected in the results. Overall, the methodological approach of involving students in the development of the curriculum can be recommended. The results can provide orientation to those involved in the development of rural health programs in other medical faculties.

\section{Acknowledgement}

We would especially like to thank Inga Beig for her great help in entering the data. 


\section{Competing interests}

The authors declare that they have no competing interests.

\section{Attachments}

Available from http://www.egms.de/en/joumals/zma/2018-35/zma001188.shtml

1. Attachment_1.pdf (149 KB)

Questionnaire: Development of an optional rural health program

\section{References}

1. Goodfellow A, Ulloa JG, Dowling PT, Talamantes E, Chheda S, Bone C, Moreno G. Predictors of primary care physician practice location in underserved urban or rural areas in the United States: A systematic literature review. Acad Med. 2016;91(9):13131321. DOI: $10.1097 /$ ACM. 0000000000001203

2. Krupa LK, Chan BT. Canadian rural family medicine training programs: growth and variation in recruitment. Can Fam Physician. 2005;51:852-853.

3. Eley DS, Synnott R, Baker PG, Chater AB. A decade of Australian rural clinical school graduates - where are they and why? Rural Remote Health. 2012;12:1937. Zugänglich unter/available from: http://www.rrh.org.au/publishedarticles/article_print_1937.pdf

4. Blozik E, Ehrhardt M, Scherer M. Förderung des allgemeinmedizinischen Nachwuchses: Initiativen in der universitären Ausbildung von Medizinstudierenden. Bundesgesundhbl Gesundheitsforsch Gesundheitsschutz. 2014;57(7):892-902. DOI: 10.1007/s00103-014-1984-6

5. Barthen L, Beig I, Sennekamp M, Gerlach FM, Erler A, RavensTaueber G. Raus aufs Land während des Medizinstudiums: Eine Übersicht zu bestehenden und geplanten allgemeinmedizinischen Förderangeboten. Z Allg Med. 2016;92(11):448-445. DOI: 10.3238/zfa.2016.0448-0455

6. Samos FA, Heise M, Fuchs S, Mittmann S, Bauer A, Klement A. A pilot phase evaluation of the elective general pracitice class: results of student surveys of the first two years. GMS J Med Educ. 2017;34(1):Doc4. DOI: 10.3205/zma001081

7. Deutsch T, Hönigschmid P, Frese T, Sandholzer H. Early community-based family practice elective positively influences medical students' career considerations - a pre-post-comparison. BMC Fam Pract. 2013;14:24. DOI: 10.1186/1471-2296-14-24

8. Schäfer H, Mangold B, Paulitsch M, Gerlach FM. Landpartie Fulda - ein Projekt zur Förderung des hausärztlichen Nachwuchses im ländlichen Raum. Z Allg Med. 2015;91(6):260-263. DOI: 10.3238/zfa.2015.0260-0263

9. Bundesvertretung der Medizinstudierenden in Deutschland (bvmd). Longitudinale Einbindung der Allgemeinmedizin in das Medizinstudium. Berlin: bvmd; 2014. Zugänglich unter/available from: https://www.bvmd.de/fileadmin/redaktion/ Positionspapiere/Positionspapier_2014-04-26_Longit.Einbindung-der-Allgemeinmed-in-Medizinstudium.pdf

10. Huenges B, Gulich M, Böhme K, Fehr F, Streitlein-Böhme I, Rüttermann V, Baum E, Niebling WB, Rusche $\mathrm{H}$. Recommendations for undergraduate training in the primary care sector -position paper of the GMA-Primary Care Committee. GMS Z Med Ausbild. 2014;31(3):Doc35. DOI: 10.3205/zma000927
11. Yengo-Kahn AM, Baker CE, Lomis KD. Medical students' perspectives on implementing curriculum change at one institution. Acad Med. 2017;92(4):455-461. DOI: 10.1097/ACM.0000000000001569

12. Atkins KM, Roberts AE, Cochran N. How medical students can bring about curricular change. Acad Med. 1998;73(11):11731176. DOI: 10.1097/00001888-199811000-00014

13. Jacob R, Kopp J, Schultz S. Berufsmonitoring Medizinstudenten 2014: Ergebnisse einer bundesweiten Befragung. Berlin: Kassenärztliche Bundesvereinigung; 2015. Zugänglich unter/available from: http://www.kbv.de/media/sp/2015_04_ 08_Berufsmonitoring_2014_web.pdf

14. Glasser M, Hunsaker M, Sweet K, MacDowell M, Meurer M. A comprehensive medical education program response to rural primary care needs. Acad Med. 2008;83(10):952-961. DOI: 10.1097/ACM.0b013e3181850a02

15. Greer T, Kost A, Evans DV, Norris T, Erickson J, McCarthy J, Allen S. The WWAMI Targeted Rural Underserved Track (TRUST) Program: an innovative response to rural physician workforce shortage. Acad Med. 2016;91(1):65-69. DOI: 10.1097/ACM.0000000000000807

16. Langosch C, Onnasch JF, Steger T, Klement A, Grundke S. Die "Klasse Allgemeinmedizin" als Wahlpflichtfach im vorklinischen Studienabschnitt: Didaktischer Aufbau, Lehrziele und Umsetzung. GMS J Med Educ. 2012;29(5):Doc67. DOI: 10.3205/zma000837

17. Broerman M, Wunder A, Sommer S, Baum E, Gerlach FM, Sennekamp M. Hessenweites Weiterbildungskolleg für Ärztinnen und Ärzte in Weiterbildung Allgemeinmedizin. Z Allg Med. 2015;91(1):18-22. DOI: 10.3238/zfa.2015.0018-0022

18. Cohen J. Statistical power analysis for the behavioral sciences. 2 ed. Hillsdale, NJ: L. Erlbaum Associates Inc.; 1988.

19. Bundesinstitut für Bau-, Stadt- und Raumforschung. Laufende Stadtbeobachtung - Raumabgrenzungen. Bonn: Bundesinstitut für Bau-, Stadt- und Raumforschung; 2015. Zugänglich unter/available from: http://www.bbsr.bund.de/BBSR/DE/ Raumbeobachtung/Raumabgrenzungen/StadtGemeindetyp/ StadtGemeindetyp_node.html

20. Bortz J, Doering N. Forschungsmethoden und Evaluation: Für Human- und Sozialwissenschaftler. 4 ed. Berlin, Heidelberg: Springer; 2006. DOI: 10.1007/978-3-540-33306-7

21. Verma P, Ford JA, Stuart A, Howe A, Everington S, Steel N. A systematic review of strategies to recruit and retain primary care doctors. BMC Health Serv Res. 2016;16(1):126. DOI: 10.1186/s12913-016-1370-1

22. Jungbauer J, Kamenik C, Alfermann D, Brähler E. Wie bewerten angehende Arzte rückblickend ihr Medizinstudium? Ergebnisse einer Absolventenbefragung. Gesundheitswesen. 2004;66(1):5156. DOI: $10.1055 / \mathrm{s}-2004-812705$

23. Bundesministerium für Bildung und Forschung. Masterplan Medizinstudium 2020. Berlin: Bundesministerium für Bildung und Forschung; 2017. Zugänglich unter/available from: https:/ /www.bmbf.de/files/2017-03-31_Masterplan\% 20Beschlusstext.pdf

\section{Corresponding author:}

Linda Barthen

Goethe University Frankfurt, Institute of General Practice, Theodor Stern Kai 7, D-60590 Frankfurt/Main, Germany barthen@allgemeinmedizin.uni-frankfurt.de 


\section{Please cite as}

Barthen L, Ravens-Taeuber G, Paulitsch MA, Gerlach FM, Sennekamp M. How can General Practice be incorporated longitudinally in medical studies? Students' views on the development of a new rural health program. GMS J Med Educ. 2018;35(3):Doc42.

DOI: 10.3205/zma001188, URN: urn:nbn:de:0183-zma0011886

This article is freely available from

http://www.egms.de/en/journals/zma/2018-35/zma001188.shtml
Received: 2017-10-12

Revised: 2018-05-17

Accepted: 2018-06-06

Published: 2018-08-15

\section{Copyright}

(C)2018 Barthen et al. This is an Open Access article distributed under the terms of the Creative Commons Attribution 4.0 License. See license information at http://creativecommons.org/licenses/by/4.0/. 


\section{Wie kann die Allgemeinmedizin longitudinal im Studium verankert werden? Die Perspektive der Studierenden auf die Gestaltung eines neuen Schwerpunktprogramms im ländlichen Raum}

\section{Zusammenfassung}

Zielsetzung: Beteiligung von Medizinstudierenden im Rahmen der konzeptionellen Entwicklung eines zielgruppenspezifischen und attraktiven allgemeinmedizinischen Lehrangebots im ländlichen Raum.

Methodik: Es wurde ein Fragebogen entwickelt, der die Bewertung der Studierenden hinsichtlich des aktuellen Ablaufs ihres Studiums, den späteren Berufswunsch sowie die Anforderungen an ein zu entwickelndes allgemeinmedizinisches Schwerpunktprogramm im ländlichen Raum erfasst. Mittels einer Online-Befragung wurden im Sommer 2015 alle Medizinstudierende ab dem vierten vorklinischen Semester $(n=2.150)$ der Goethe-Universität Frankfurt einmalig befragt. Die statistische Auswertung erfolgte primär deskriptiv. Die persönliche Einstellung hinsichtlich der Bereitschaft, als Hausarzt tätig zu werden, wurde auf statistische Signifikanz überprüft. Zudem wurde erhoben, ob ein messbarer Zusammenhang zwischen der eigenen Herkunft und dem späteren Wunscharbeitsort besteht.

Ergebnisse: Von insgesamt 2.150 kontaktierten Studierenden nahmen 617 an der Befragung teil (Rücklaufquote=28,7\%). Die Ergebnisse repräsentieren eine große Bandbreite an Ideen und Anregungen, die sowohl die Meinung von Befürwortern als auch eher kritisch gegenüber der Lehre in der Allgemeinmedizin eingestellten Medizinstudierenden widerspiegeln. Von dem geplanten Schwerpunktprogramm erwarten die Studierenden einen starken Praxisbezug ebenso wie das Kennenlernen administrativer sowie wirtschaftlicher Hintergründe zum Führen einer Praxis.

Schlussfolgerungen: Durch die Einbeziehung der Zielgruppe am Entwicklungsprozess bestand die Möglichkeit, das zu entwickelnde Schwerpunktprogramm auf die späteren Teilnehmer passgenauer zuzuschneiden. Zudem ist zu erwarten, dass die Beteiligung der Studierenden zu einer höheren Akzeptanz des Programms führt. Die gewonnenen Ergebnisse zur Gestaltung eines Lehrangebots können als Orientierung für die mögliche Entwicklung ähnlicher Schwerpunktprogramme an anderen medizinischen Fakultäten dienen.

Schlüsselwörter: Allgemeinmedizin, Medizinstudierende, Curriculum, Landarztprogramm, Hausarztmangel

\author{
Linda Barthen ${ }^{1}$ \\ Gisela Ravens-Taeuber ${ }^{1}$ \\ Michael A. Paulitsch ${ }^{1}$ \\ Ferdinand M. Gerlach ${ }^{1}$ \\ Monika Sennekamp ${ }^{1}$ \\ 1 Goethe-Universität Frankfurt, \\ Institut für Allgemeinmedizin, \\ Frankfurt/Main, Deutschland
}

\section{Einleitung}

Vor dem Hintergrund eines zunehmenden Mangels an Hausärzten, vor allem in den ländlichen Regionen Deutschlands, stellt die Gewinnung des medizinischen Nachwuchses eine essentielle Herausforderung dar. Die Förderung der Allgemeinmedizin in Form von Schwerpunktangeboten während des Medizinstudiums kann einen positiven Effekt auf die Bereitschaft haben, nach dem
Studium hausärztlich (in ländlichen Gebieten) tätig zu werden [1]. Im Ausland, vor allem in den USA, Kanada und Australien, existieren bereits zahlreiche Schwerpunktprogramme, die zum Ziel haben, die Allgemeinmedizin während der Ausbildung zu fördern [2], [3]. Auch in Deutschland wächst die Zahl solcher Angebote [4], [5]. Erste Hinweise deuten darauf, dass der Kompetenzerwerb im Rahmen allgemeinmedizinischer Programme einen positiven Einfluss auf die Präferenz zur Fachgebietswahl Allgemeinmedizin hat [6] bzw. das Fach als erste Karriereoption gefördert wird [7]. 
Am Fachbereich Medizin in Frankfurt am Main gibt es seit 2012 das Angebot der „Landpartie Fulda“: Ein freiwilliges Förderprogramm bei dem die teilnehmenden Studierenden ihr Blockpraktikum Allgemeinmedizin in ausgewählten Landarztpraxen im Landkreis Fulda in Hessen absolvieren. Es konnte belegt werden, dass die Teilnahme am Programm zu einem signifikanten Motivationszuwachs, später hausärztlich tätig zu werden, führt [8]. Wie von Fachvertretern und Studierenden gefordert, sollte die Allgemeinmedizin zukünftig jedoch auch longitudinal im Studium verankert werden [9], [10]. Daher sollte in Kooperation mit dem Studiendekanat zusätzlich zu dem bereits bestehenden Angebot der „Landpartie Fulda“ ein über mehrere Semester laufendes und auf weitere Landkreise ausgedehntes Programm konzipiert werden. Bei curricularen Änderungen oder Neuerungen dieser Art sind Studierende allerdings selten involviert. Dabei konnten Studien belegen, dass die Beteiligung der Zielgruppe selbst eine Kernvoraussetzung für den Erfolg und die Akzeptanz einer Curriculumsänderung ist [11]. Die Studierenden als Experten in eigener Sache wissen um die Defizite und Potenziale ihres bisherigen Studienplans und sind zudem hoch motiviert, am Veränderungsprozess mitzuwirken [12].

Ziel der hier dargelegten Erhebung war es daher, die Studierenden in die konzeptionelle Entwicklung eines neuen „Landarztprogramms“ (folgend auch „Schwerpunktprogramm“) miteinzubeziehen. Im Mittelpunkt stand die Frage, welche Wünsche und Anforderungen die Studierenden selbst an ein Schwerpunktprogramm, das die Allgemeinmedizin im ländlichen Raum fördert, stellen. Zudem wurden der Berufswunsch sowie die Bewertung der Allgemeinmedizin im aktuellen Studium erfasst.

\section{Methoden}

\section{Datenerhebung}

Die Einladung zur Beteiligung an einer webbasierten Befragung im Querschnittsdesign wurde an alle Studierenden ab dem 4. vorklinischen Semester der Goethe-Universität Frankfurt verschickt. Die Kontaktaufnahme erfolgte per E-Mail-Verteiler durch das Dekanat und das Institut für Allgemeinmedizin. Die Auswahl der Studienpopulation basierte zum einen auf der Annahme, dass Studierende erst ab dem 4. vorklinischen Semester ausreichend über die Studienstrukturen und den weiteren Studienverlauf informiert sind. Zudem lagen für diese Jahrgänge E-MailVerteiler vor, sodass eine Ansprache direkt möglich war. Die E-Mail enthielt ein kurzes Informationsschreiben sowie den zur Online-Befragung führenden Link. Insgesamt wurden 2.150 Studierende erstmals Ende Mai 2015 kontaktiert, was der Grundgesamtheit entsprach. Ein Erinnerungsschreiben folgte Mitte Juni 2015.

\section{Erhebungsinstrument}

Der selbstkonzipierte Fragebogen (siehe Anhang 1) wurde auf Grundlage der Ergebnisse aus einer bundesweiten Befragung von Medizinstudierenden aus dem Jahr 2015 erstellt [13] und in einem interdisziplinären Team bestehend aus einer Ärztin in Weiterbildung, einem erfahrenen Hausarzt, einer Pädagogin, einem Psychologen und einer Gesundheitswissenschaftlerin weiterentwickelt sowie abgestimmt.

Der Fragebogen wurde in einem Pretest von drei Studierenden geprüft und anschließend angepasst. In seiner Endversion enthielt der Fragebogen insgesamt 19 Items zu den Themenbereichen Sozio-demographische Angaben, Berufswunsch und Studium sowie Angaben zu dem zu entwickelnden Schwerpunktprogramm. Das Interesse im Studienverlauf an einer hausärztlichen Niederlassung wurde anhand einer sechsstufigen Ratingskala erfasst (1=,Trifft voll zu“; 6=„Trifft überhaupt nicht zu“). Neben überwiegend geschlossenen Fragen gab es fünf offene Fragen im Bogen.

Im letzten Themenbereich wurde den Befragten eingangs eine erste Konzeptidee des zukünftigen Programms auf Basis der Literatur bzw. bereits andernorts etablierter Programmen beschrieben (Bestandteile: Praxisphasen, Begleitendes Seminar- und Mentoringprogramm) [z.B. [14], [15], [16], [17]. Die Teilnehmer sollten die skizzierte Idee anschließend in einzelnen Fragen kommentieren und abschließend angeben, ob sie selbst an solch einem Angebot teilnehmen würden.

Für die Befragung wurde das Umfrage-Tool Survey Monkey gewählt, um einen niederschwelligen Zugang zu ermöglichen. Dadurch sollten auch Studierende erreicht werden, die bisher keinen oder wenig Kontakt zur Allgemeinmedizin hatten oder kritisch gegenüber allgemeinmedizinischer Lehre eingestellt sind.

Die aus der Befragung abgeleiteten Ansätze für das Schwerpunktprogramm wurden im Nachgang mit einzelnen Studierenden aus der Fachschaft in einer Gesprächsrunde final abgestimmt.

\section{Datenauswertung}

Die Auswertung der Daten erfolgte primär deskriptiv mittels der Statistiksoftware IBM SPSS. Für die kategorialen Variablen wurden die absoluten und relativen Häufigkeiten ermittelt. Potentielle Mittelwertunterschiede hinsichtlich der Bereitschaft als Hausarzt tätig zu werden, wurden anhand eines gepaarten Wilcoxon-Tests auf statistische Signifikanz hin überprüft. Zur Quantifizierung möglicher Unterschiede wurde die Effektstärke von Cohens d berechnet: 0,2-0,4=kleiner Effekt; 0,5-0,8=mittlerer Effekt und $>0,8=$ großer Effekt [18]. Zudem wurde die Korrelation nach Spearman zwischen der eigenen Herkunft und dem späteren Wunscharbeitsort überprüft ( $\alpha \leq 5 \%)$.

Bei der Darstellung der sozio-demographischen Angaben wurde die ursprünglich sechsstufige Ratingskala der eigenen Herkunft auf die dichotome Skalierung Landgemeinde und Stadt reduziert [19]. 
Die offenen Antworten wurden mittels einer quantitativen Inhaltsanalyse ausgewertet. Bei dieser Methode werden die relevanten Merkmale der Textpassagen erfasst, indem sie einem entwickelten Kategoriensystem zugeordnet und somit operationalisiert werden. Die Häufigkeiten in den einzelnen Kategorien können dann als Indikator für die Eigenschaften der Texte dienen [20]. In der Befragung antworteten die Teilnehmer in der Regel stichpunktartig oder in ganzen Sätzen. Einzelne Stichpunkte wurden als eine Einheit (z.B. Frage 13 a:„Eigene Patienten zur Voruntersuchung“), längere Stichpunkte oder mehrere Sätze je nach Inhalt in mehreren Einheiten erfasst (z.B. Frage $13 \mathrm{a}$ „Praxis sollte mind. einen freien Untersuchungsraum für den Studenten zu Verfügung haben, außerdem wäre die Möglichkeit apparativer Diagnostik zu üben schön“). Die einzelnen Einheiten wurden dann den Kategorien zugeordnet (z.B. „Eigene Patienten zur Voruntersuchung “=Praktisch tätig sein; „Praxis sollte mind. einen freien Untersuchungsraum für den Studenten zu Verfügung haben “=Eigener Behandlungsraum; „....apparativer Diagnostikzu üben... “=Kennenlernen und Anwenden der Diagnosetechniken), die zuvor anhand des vorliegenden Textmaterials abgeleitet wurden (induktiv geleitetes Vorgehen). Die Auswertung der offenen Antworten erfolgte durch zwei Kodiererinnen. Bei der Darstellung der Ergebnisse wurden nur Kategorien mit mindestens zehn Nennungen berücksichtigt (vgl. Tabelle 1).

\section{Ergebnisse}

Nach einer Datenbereinigung konnten 617 Fragebögen für die Auswertung berücksichtigt werden (Rücklaufquote $=28,7 \%$ ). Unter den Teilnehmern waren 423 weiblich (68,6\%). Das mittlere Geburtsjahr betrug 1990 (Range: 1968-1995) und im Schnitt befanden sich die Teilnehmer im 8. Fachsemester (Range: 2.-16. Fachsemester; Eine Person gab an, sich erst im 2. Fachsemester zu befinden). Die Mehrzahl (76,0\%) ist in einer Stadt (>5.000 Einwohner) aufgewachsen. Circa ein Fünftel (21,0\%) entstammt dagegen einer Landgemeinde (<5.000 Einwohner). Ferner gibt knapp ein Fünftel (18,5\%) an, über eine abgeschlossene Berufsausbildung zu verfügen. Lediglich 4,5\% der Befragten haben Kinder.

\section{Aktuelle Studiensituation und Berufswunsch}

Danach befragt, in welchem Abschnitt des Medizinstudiums Studierende erstmalig praktische Erfahrungen in einer hausärztlichen Praxis sammeln sollten, spricht sich insgesamt knapp ein Viertel der Befragten (23,5\%) für die Vorklinik aus. Die deutliche Mehrheit mit knapp zwei Dritteln $(65,8 \%)$ hält dagegen den klinischen Studienabschnitt für geeignet. Lediglich 5,4\% befürworten ein Praktikum vor Beginn des Studiums. Unterschiede zeigen sich hier zwischen der Vorklinik und Klinik. Während 41,7\% der Studierenden aus dem vorklinischen Studienabschnitt selbigen als geeigneten Zeitpunkt zum Erstkontakt mit der hausärztlichen Praxis einstufen, teilen diese
Einschätzung nur 17,6\% der Studierenden ab dem klinischen Abschnitt.

Die bisherigen Erfahrungen mit dem Fach Allgemeinmedizin wurden in der Befragung nicht erfasst, dürften zwischen den Teilnehmer jedoch variieren in Abhängigkeit des jeweiligen Studienjahres.

Unter der Annahme, dass eine spätere hausärztliche Tätigkeit in Frage kommt, sollten die Studierenden weiterhin angeben, ob sie sich hierzu durch ihre bisherige Ausbildung ausreichend vorbereitet fühlen. Die Frage wird mit zunehmendem Fachsemester bejaht. Dennoch geben $19,0 \%$ aller Befragten ab dem 11. Fachsemester $(\mathrm{N}=158)$ an, dass sie sich nicht ausreichend vorbereitet fühlen bzw. 55,1\% sagen, sich nur zum Teil ausreichend vorbereitet zu fühlen (insgesamt 74,1\%). Voraussetzungen für eine ausreichende Vorbereitung sind aus Sicht der Befragten ( $\mathrm{N}=97$; offene Frage): Mehr Praxis (53,6\%), Administrative und wirtschaftliche Hintergründe einer Praxis kennen (15,5\%) sowie Mehr fächerübergreifendes Wissen (11,4\%).

Auch das grundsätzliche Interesse an einer allgemeinmedizinischen Tätigkeit wurde erfasst. Anhand einer sechsstufigen Ratingskala (1=,,Trifft voll zu“; 6= ,Trifft überhaupt nicht zu“) wurden die Teilnehmer gefragt, "Ob sie sich zu Beginn des Studiums vorstellen konnten, als niedergelassener Hausarzt zu arbeiten“ (Mittelwert=3,81) und „Ob sie sich jetzt vorstellen können, später als niedergelassener Hausarzt zu arbeiten “(Mittelwert=3,36). Ein Vergleich der Werte mittels des Wilcoxon-Tests zeigt, dass das Interesse im Laufe des Studiums statistisch signifikant zunimmt $(p<0,001 ; N=617)$. Anhand von $d$ nach Cohen zeigt sich mit 0,278 ein kleiner Effekt [18]. Ferner geben 43,5\% $(n=268)$ der Studierenden an, sich eher nicht bis überhaupt nicht vorstellen zu können, später als Allgemeinmediziner zu arbeiten. Dennoch haben hiervon beispielsweise 46,7\% ( $n=125)$ konkrete Vorschläge und Ideen zur Ausgestaltung der Praxisphasen gegeben, die in den Ergebnissen in Tabelle 1 mündeten.

In Abbildung 1 ist die präferierte Anzahl an Einwohnern des späteren Arbeitsortes angegeben. Zwischen den Variablen der eigenen Herkunft und des Wunscharbeitsortes zeigt sich anhand einer Korrelation eine mittlere Effektstärke bei statistischer Signifikanz $(r=0,366$; $p<0,001$; $\mathrm{N}=458$ ) [20]. Knapp ein Drittel der Teilnehmer, die aus Landgemeinden stammen, gibt an, noch keine Präferenz für den späteren Arbeitsort zu haben.

\section{Angaben zu dem zu entwickelnden Schwerpunktprogramm}

Zur Ausgestaltung des Schwerpunktprogramms wurde den Studierenden eine erste Konzeptidee skizziert (Bestandteil I: Praxisphasen; Bestandteil II: Begleitendes Seminar; Bestandteil III: Mentoringprogramm). Etwa die Hälfte der Teilnehmer nutzte die Gelegenheit, Anforderungen an das allgemeinmedizinische Schwerpunktprogramm und eigene Ideen in Form von Freitextantworten zu formulieren. 
Tabelle 1: Angaben zu dem zu entwickelnden Schwerpunktprogramm in den beschriebenen drei Bestandteilen (Auswertung der offenen Fragen; Darstellung von Kategorien, mit mind. 10 Nennungen).

\begin{tabular}{|c|c|c|c|}
\hline \multicolumn{4}{|c|}{ PRAXISPHASEN BEIM HAUSARZT, $N=329$} \\
\hline & & $\mathbf{n}$ & $\%$ \\
\hline - & Aktiver Praxisbezug & 207 & 62,9 \\
\hline$\cdot$ & Regelmäßiges und ausführliches Feedback durch den Lehrarzt & 115 & 35,0 \\
\hline & Organisation und Rahmenbedingungen & & \\
\hline & $\circ$ Eigener Behandlungsraum & 22 & 6,7 \\
\hline & 1:1 Betreuung & 19 & 5,8 \\
\hline & Motivierte/engagierte Ärzte/gute Betreuung & 15 & 4,6 \\
\hline & Freie Praxiswahl & 12 & 3,7 \\
\hline & $\circ$ Organisation der An- und Abreise & 12 & 3,7 \\
\hline & $\begin{array}{l}\text { Kennenlernen und Anwenden der Diagnosetechniken (EKG, Sonographie, } \\
\text { Lungenfunktion) }\end{array}$ & 29 & 8,8 \\
\hline & $\begin{array}{l}\text { Kennenlernen des erweiterten Tätigkeitsspektrums (Haus- und Pflegeheimbesuche, } \\
\text { Begleitung der MFAs, Teilnahme am Ärttlichen Bereitschaftsdienst) }\end{array}$ & 27 & 8,2 \\
\hline & $\begin{array}{l}\text { Praxismanagement (EDV, Abrechnungen, Kodierung, Aktendokumentation etc.) und } \\
\text { Praxisalltag kennenlernen }\end{array}$ & 21 & 6,4 \\
\hline \multicolumn{4}{|c|}{ SEMINARE, $N=303$} \\
\hline & Themenvorschläge für Seminare & & \\
\hline & $\begin{array}{l}\text { - Praktische Übungen - Anamnese bis Therapieplanung anhand typischer } \\
\text { Krankheitsbilder, Fallbesprechungen vor den Praktika }\end{array}$ & 129 & 42,6 \\
\hline & - Erfahrungsaustausch, Fallbesprechungen nach den Praktika & 88 & 29,0 \\
\hline & Praxismanagement (EDV, Abrechnungen, Kodierung, Aktendokumentation etc.) und & 30 & 9,9 \\
\hline & Praxisformen kennenlernen & & 9,2 \\
\hline & - Praktische Übungen - Diagnostik (Sonographie, EKG, Labor, Wundversorgung etc.) & 28 & \\
\hline & $\begin{array}{l}\text { Vermittlung von Soft Skills/Sozialen Kompetenzen/Kommunikationstraining/Umgang } \\
\text { mit (schwierigen) Patienten und Situationen }\end{array}$ & 21 & 6,9 \\
\hline & ○ Notfallversorgung; Überweisung an Fachärzte & & \\
\hline & ○ Vergleich Stadt/Land; Darstellung der Vor- und Nachteile einer hausärztlichen & & 3,7 \\
\hline & Tätigkeit auf dem Land & $\begin{array}{l}12 \\
12\end{array}$ & 3,7 \\
\hline & Organisation und Rahmenbedingungen & & \\
\hline & Keine/wenige Referate durch Studierende & 27 & 8,9 \\
\hline & $\begin{array}{l}\text { - Interaktiv, wenig/nicht frontal, Workshops, Themenauswahl gemeinsam mit } \\
\text { Studierenden }\end{array}$ & 20 & 6,6 \\
\hline & Sehr kleine Gruppen & 20 & 6,6 \\
\hline & $\circ$ Echte Patienten/echte Fälle/Schauspielpatienten & 20 & 6,6 \\
\hline \multicolumn{4}{|c|}{ MENTORING, N = 259} \\
\hline & Hausarzt als Ansprechpartner/Mentor & & \\
\hline & - Vertrauensvoller Ansprechpartner bei Fragen jeglicher Art & 65 & 25,1 \\
\hline & Tipps/Gezielte Anleitungen in der Praxis und Feedback & 61 & 23,6 \\
\hline & Beratung zu beruflichen/persönlichen Werdegang & 21 & 8,1 \\
\hline & - Gemeinsame Fallbesprechung/Besprechung von Therapiestrategien & 18 & \\
\hline & $\begin{array}{l}\text { Entwicklung praktischer Fertigkeiten (Praktische Erfahrungen sammeln/Anwendung von } \\
\text { Wissen, eigenständiges Arbeiten, erlernen von/sicherer Umgang mit Patienten) }\end{array}$ & 70 & 27,0 \\
\hline & Organisation und Rahmenbedingungen & & \\
\hline & - Gute 1:1 Betreuung/Kleingruppen & 32 & 12,4 \\
\hline & - Gute Erreichbarkeit des Mentors & 30 & 11,6 \\
\hline & $\circ \quad$ Motivierte und lehrfähige Mentoren mit ausreichend Zeit & 28 & 10,8 \\
\hline & $\begin{array}{l}\text { Realistischer Einblick in Praxismanagement (EDV, Abrechnungen, Kodierung, } \\
\text { Aktendokumentation etc.) und Praxisalltag }\end{array}$ & 38 & 14,7 \\
\hline & Von langjähriger Berufserfahrung des Hausarztes profitieren & 27 & 10,4 \\
\hline
\end{tabular}

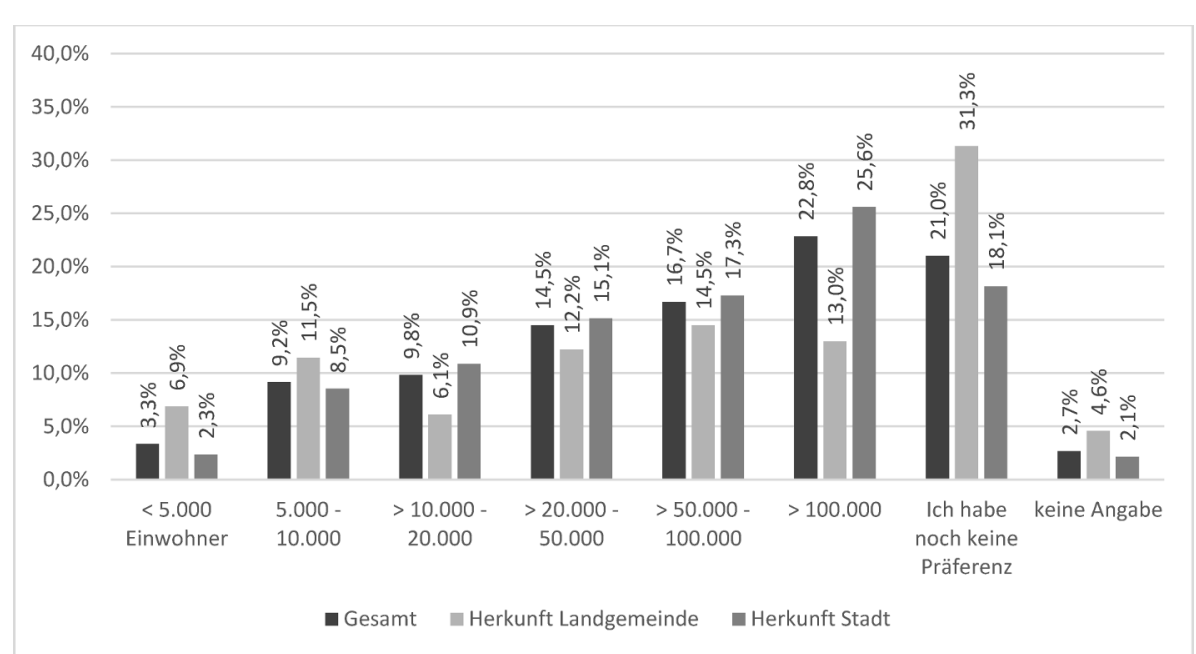

Abbildung 1: Präferierte Einwohnerzahl des späteren Arbeitsortes nach der eigenen Herkunft, N=617.

Als wichtigste Voraussetzung für die Praxisphasen beim Hausarzt (Bestandteil I) wird von 62,9\% der Teilnehmer der Aktive Praxisbezug genannt. Hierunter fällt vor allem die selbstständige Behandlung eigener Patienten von der Anamneseerhebung, über die Diagnostik bis hin zur Therapieempfehlung unter (anfänglicher) Supervision. 
Daneben wünscht sich über ein Drittel der Befragten $(35,0 \%)$ ein regelmäßiges und ausführliches Feedback durch den Lehrarzt. Bei den begleitenden Kleingruppenseminaren an der Universität (Bestandteil II) werden vorrangig konkrete Themen für die inhaltliche Gestaltung des Seminars genannt. Als besonders bedeutsam werden dabei Praktische Übungen (42,6\%) sowie Erfahrungsaustausch und Fallbesprechungen nach den Praxisphasen (29,0\%) eingestuft. Von einem Mentoring (Bestandteil III) erwarten sich die Teilnehmer einen Hausarzt, der Ihnen fachliche/praktische Tipps und Feedback gibt $(23,6 \%)$ sowie als Ansprechpartner bei Fragen jeglicher Art (25,1\%) zur Seite steht. Durch das Mentoring erhoffen sich die Studierenden zudem die Entwicklung praktischer Fertigkeiten beispielsweise in Form von Praktika (27,0\%). Die vollständigen Ergebnisse sind in Tabelle 1 abgebildet. Befragt nach der gewünschten Dauer des Schwerpunktprogramms gehen die Antworten auseinander (Antworten waren vorgegeben). Über ein Viertel $(27,7 \%)$ spricht sich für ein Semester aus. Jeweils ein weiteres Viertel wünscht sich eine Dauer von mehreren Semestern (23,0\%) oder hat keine Vorgaben solange „das Angebot für mich sinnvoll ist“ (23,5\%). Weniger als $5 \%$ wünschen sich ein Schwerpunktprogramm, das das gesamte Studium umfasst.

Abschließend wurden die Teilnehmer gefragt, ob sie an dem skizzierten Angebot teilnehmen würden ( $\mathrm{Ja}=45,4 \%$; Nein=17,5\%; Weiß nicht=30,6\%; keine Angabe=6,5\%). Diejenigen, die sich gegen eine Teilnahme aussprechen, begründen dies mit kein Interesse an der Allgemeinmedizin (61,5\%), kein Interesse, in einer ländlichen Region tätig zu werden (45,0\%), keine Zeit im Studium (41,3\%), kein Interesse an einer Niederlassung (21,1\%) sowie kein Interesse an weiteren Praktika (21,1\%).

\section{Diskussion}

617 Medizinstudierende ab dem 4. vorklinischen Semester nutzten die Online-Befragung, um sich an der Entwicklung eines allgemeinmedizinischen Schwerpunktprogramms für den ländlichen Raum zu beteiligen.

Insbesondere der Bedarf an mehr Praxis im Studium spiegelt sich in den Ergebnissen der vorliegenden Untersuchung bzw. den formulierten Anforderungen an das zu entwickelnde Lehrangebot wider. In allen drei Kernbereichen (Praxisphasen, begleitendes Seminar- und Mentoringprogramm) fordern die potentiellen Teilnehmer einen starken Praxisbezug. Der Wunsch, eigene Patienten zu behandeln, die persönlichen Fähigkeiten weiterzuentwickeln und aktiv in den Praxisalltag einbezogen zu werden, wird jeweils auf die offenen Fragen angegeben. Darüber hinaus spielen das Kennenlernen des Praxismanagements sowie der Praxisorganisation eine wichtige Rolle und stellen somit einen Bereich dar, der im Schwerpunktprogramm Beachtung finden sollte.

Unabhängig vom Fach präferiert über die Hälfte der Befragten (54,0\%) eine spätere Tätigkeit in einer Mitteloder Großstadt (>20.000 Einwohner). Ob dies „ein Woh- nen in der Stadt und ein Arbeiten auf dem Land" als mögliches Zukunftsmodell ausschließt, bleibt allerdings unklar. Dass Studierende mit einer ländlichen Herkunft eher bereit sind, dorthin zurückzukehren ist zudem bereits mehrfach beschrieben worden [1], [21]. Dass jedoch knapp ein Drittel (31,3\%) der Studierenden aus Landgemeinden hinsichtlich des später gewünschten Arbeitsorts unentschlossen ist, weist auf das Potential hin, diese Zielgruppe für die Teilnahme an dem Schwerpunktprogramm anzusprechen und somit für einen entsprechenden Werdegang frühzeitig zu begeistern.

Knapp dreiviertel aller Teilnehmer (74,1\%) ab dem 11. Fachsemester fühlen sich nicht oder nur unzureichend auf eine mögliche hausärztliche Tätigkeit vorbereitet. Gründe hierfür sind vorrangig fehlende praktische Erfahrungen, aber auch unzureichende Kenntnisse über die administrativen sowie wirtschaftlichen Voraussetzungen zum Führen einer Praxis. Die Ergebnisse stellen per se keinen Grund zur Beunruhigung dar, da erst die Facharztweiterbildung zum Allgemeinmediziner diese Anforderungen in Gänze erfüllen sollte. Das Studium verfolgt hingegen das Ziel „...grundlegende Kenntnisse, Fähigkeiten und Fertigkeiten in allen Fächern zu vermitteln, die für eine umfassende Gesundheitsversorgung der Bevölkerung erforderlich sind." [https://www.gesetze-im-internet.de/_appro_2002/BJNR240500002.html]. Dennoch konnte eine Absolventenbefragung zeigen, dass der mangelnde Praxisbezug und die unzureichende Vermittlung praktischer, ärztlicher Fähigkeiten als defizitäre Aspekte des Medizinstudiums wahrgenommen werden [22]. Auch die Politik hat diese Probleme erkannt und versucht nun, im Rahmen des Masterplans Medizinstudium 2020, aktiv dagegen anzugehen. So sieht der Masterplan vor, nicht nur den allgemeinen Praxisanteil im Studium zu erhöhen, sondern insbesondere auch die Ausbildung im hausärztlichen Versorgungssetting zu stärken [23].

Die Untersuchung konnte zudem zeigen, dass das Interesse der Studierenden an einer möglichen allgemeinmedizinischen Tätigkeit im Studienverlauf zunimmt. Zu ähnlichen Ergebnissen kommt auch eine Studie aus dem Jahr 2015 von Jacob, Kopp und Schultz [13]. Weiterhin ist anzumerken, dass sich 45,4\% der Antwortenden eine Teilnahme am skizzierten Programm vorstellen können, obwohl dieses zum Befragungszeitpunkt nur in groben Umrissen beschrieben werden konnte und es sich darüber hinaus um ein sehr fokussiertes Schwerpunktprogramm handeln wird (Allgemeinmedizin im ländlichen Raum). Zwar kann nicht davon ausgegangen werden, dass dies der tatsächlichen Nachfrage entsprechen wird, dennoch wird hier frühzeitig ein Bedarf deutlich, der die Sinnhaftigkeit eines solchen Programms bekräftigt.

Die vorliegenden Ergebnisse müssen mit einigen Einschränkungen interpretiert werden. Der Fragebogen wurde für die Zwecke der Studie entwickelt und ist daher kein bereits validiertes Instrument. Ferner sollte bedacht werden, dass in einer Querschnittserhebung Veränderungen im „Studienverlauf“ nicht längsschnittlich bei identischen Studierenden untersucht werden können, sodass 
Unterschiede zwischen verschiedenen Jahrgangskohorten auch auf hier nicht erfasste Einflüsse zurückgeführt werden könnten. Mit Blick auf die Übertragbarkeit des Vorgehens und des entwickelten Konzepts auf andere deutsche Studienstandorte ist darauf zu achten, dass sich die Rahmenbedingungen zwischen den Universitäten voneinander unterscheiden können. Die vorliegenden Ergebnisse sind somit nicht repräsentativ für Deutschland und eine Umsetzung andernorts müsste den entsprechenden Gegebenheiten angepasst werden. Eine Stärke der Studie ist hingegen in der Beteiligung der Studierenden an der Entwicklung eines curricularen Angebots zu sehen. Es konnte gezeigt werden, dass auch Studierende, die der Allgemeinmedizin kritisch gegenüberstehen bzw. kein Interesse an einer allgemeinmedizinischen Tätigkeit haben, an der Befragung teilgenommen haben. Dadurch konnte ein möglichst breites Stimmungsbild eingefangen werden und war in diesem Fall der Methode von Fokusgruppen vorzuziehen, da so der Effekt vermieden wurde, dass nur besonders (an der Allgemeinmedizin) Interessierte in die Entwicklung eingeschlossen wurden. Um dennoch eine Feedbackschleife von den Studierenden zu erhalten, wurde das entwickelte Konzept im Nachgang mit einzelnen Studierenden aus der Fachschaft in einer Gesprächsrunde final vorgestellt und abgestimmt.

\section{Schlussfolgerungen}

Insgesamt hat die Beteiligung der Studierenden dazu beigetragen, die inhaltliche, organisatorische sowie thematische Gestaltung des Landarztprogramms zu konkretisieren. Erste Ideen konnten weiterentwickelt, neue Ansätze generiert und Hinweise berücksichtigt werden, die zuvor nicht bedacht wurden. Anhand der Ergebnisse wurde beispielsweise entschieden, dass das Schwerpunktprogramm erst ab dem klinischen Studienabschnitt startet und nicht das gesamte Studium umfasst. Ferner werden Themen zur Praxisführung und zum Praxismanagement Bestandteile der Seminarreihe sein. Im Kern wird und muss das Programm zudem einen kontinuierlichen Praxisbezug aufweisen, der sich auch in den Seminaren in Form von praktischen Übungen zeigen wird.

Es ist zudem zu erwarten, dass die Einbeziehung der Zielgruppe zu einer höheren Akzeptanz des Programms bei derselbigen führt. Für die Zukunft könnte jedoch überlegt werden, wie die Rückkopplung zu den Studierenden intensiviert und verbessert werden könnte, um gewonnene Ergebnisse nochmals zu spiegeln. Insgesamt kann das methodische Vorgehen, Studierende bei der Curriculumsentwicklung einzubeziehen, empfohlen werden. Die gewonnenen Ergebnisse können als Orientierung für die Entwicklung weiterer Schwerpunktprogramme an anderen medizinischen Fakultäten dienen.

\section{Danksagung}

Wir danken insbesondere Inga Beig, die uns bei der Dateneingabe tatkräftig unterstützt hat.

\section{Interessenkonflikt}

Die Autoren erklären, dass sie keine Interessenkonflikte im Zusammenhang mit diesem Artikel haben.

\section{Anhänge}

Verfügbar unter

http://www.egms.de/en/joumals/zma/2018-35/zma001188.shtml

1. Anhang_1.pdf (152 KB)

Fragebogen: Entwicklung eines fakultativen

Landarztprogramms/Schwerpunktprogramms

\section{Literatur}

1. Goodfellow A, Ulloa JG, Dowling PT, Talamantes E, Chheda S, Bone C, Moreno G. Predictors of primary care physician practice location in underserved urban or rural areas in the United States: A systematic literature review. Acad Med. 2016;91(9):13131321. DOI: 10.1097/ACM.0000000000001203

2. Krupa LK, Chan BT. Canadian rural family medicine training programs: growth and variation in recruitment. Can Fam Physician. 2005;51:852-853.

3. Eley DS, Synnott R, Baker PG, Chater AB. A decade of Australian rural clinical school graduates - where are they and why? Rural Remote Health. 2012;12:1937. Zugänglich unter/available from: http://www.rrh.org.au/publishedarticles/article_print_1937.pdf

4. Blozik E, Ehrhardt M, Scherer M. Förderung des allgemeinmedizinischen Nachwuchses: Initiativen in der universitären Ausbildung von Medizinstudierenden. Bundesgesundhbl Gesundheitsforsch Gesundheitsschutz. 2014;57(7):892-902. DOI: 10.1007/s00103-014-1984-6

5. Barthen L, Beig I, Sennekamp M, Gerlach FM, Erler A, RavensTaueber G. Raus aufs Land während des Medizinstudiums: Eine Übersicht zu bestehenden und geplanten allgemeinmedizinischen Förderangeboten. Z Allg Med. 2016;92(11):448-445. DOI: 10.3238/zfa.2016.0448-0455

6. Samos FA, Heise M, Fuchs S, Mittmann S, Bauer A, Klement A. A pilot phase evaluation of the elective general pracitice class: results of student surveys of the first two years. GMS J Med Educ. 2017;34(1):Doc4. DOI: 10.3205/zma001081

7. Deutsch T, Hönigschmid P, Frese T, Sandholzer H. Early community-based family practice elective positively influences medical students' career considerations - a pre-post-comparison. BMC Fam Pract. 2013;14:24. DOI: 10.1186/1471-2296-14-24

8. Schäfer H, Mangold B, Paulitsch M, Gerlach FM. Landpartie Fulda - ein Projekt zur Förderung des hausärztlichen Nachwuchses im ländlichen Raum. Z Allg Med. 2015;91(6):260-263. DOI: 10.3238/zfa.2015.0260-0263

9. Bundesvertretung der Medizinstudierenden in Deutschland (bvmd). Longitudinale Einbindung der Allgemeinmedizin in das Medizinstudium. Berlin: bvmd; 2014. Zugänglich unter/available from: https://www.bvmd.de/fileadmin/redaktion/ Positionspapiere/Positionspapier_2014-04-26_Longit.Einbindung-der-Allgemeinmed-in-Medizinstudium.pdf 
10. Huenges B, Gulich M, Böhme K, Fehr F, Streitlein-Böhme I, Rüttermann V, Baum E, Niebling WB, Rusche $H$. Recommendations for undergraduate training in the primary care sector-position paper of the GMA-Primary Care Committee. GMS Z Med Ausbild. 2014;31(3):Doc35. DOI: 10.3205/zma000927

11. Yengo-Kahn AM, Baker CE, Lomis KD. Medical students' perspectives on implementing curriculum change at one institution. Acad Med. 2017;92(4):455-461. DOl: 10.1097/ACM.0000000000001569

12. Atkins KM, Roberts AE, Cochran N. How medical students can bring about curricular change. Acad Med. 1998;73(11):11731176. DOI: 10.1097/00001888-199811000-00014

13. Jacob R, Kopp J, Schultz S. Berufsmonitoring Medizinstudenten 2014: Ergebnisse einer bundesweiten Befragung. Berlin: Kassenärztliche Bundesvereinigung; 2015. Zugänglich unter/available from: http://www.kbv.de/media/sp/2015_04 08_Berufsmonitoring_2014_web.pdf

14. Glasser M, Hunsaker M, Sweet K, MacDowell M, Meurer M. A comprehensive medical education program response to rural primary care needs. Acad Med. 2008;83(10):952-961. DOI: 10.1097/ACM.0b013e3181850a02

15. Greer T, Kost A, Evans DV, Norris T, Erickson J, McCarthy J, Allen S. The WWAMI Targeted Rural Underserved Track (TRUST) Program: an innovative response to rural physician workforce shortage. Acad Med. 2016;91(1):65-69. DOI: 10.1097/ACM.0000000000000807

16. Langosch C, Onnasch JF, Steger T, Klement A, Grundke S. Die "Klasse Allgemeinmedizin" als Wahlpflichtfach im vorklinischen Studienabschnitt: Didaktischer Aufbau, Lehrziele und Umsetzung. GMS J Med Educ. 2012;29(5):Doc67. DOI: 10.3205/zma000837

17. Broerman M, Wunder A, Sommer S, Baum E, Gerlach FM, Sennekamp M. Hessenweites Weiterbildungskolleg für Ärztinnen und Ärzte in Weiterbildung Allgemeinmedizin. Z Allg Med. 2015;91(1):18-22. DOI: 10.3238/zfa.2015.0018-0022

18. Cohen J. Statistical power analysis for the behavioral sciences. 2 ed. Hillsdale, NJ: L. Erlbaum Associates Inc.; 1988.

19. Bundesinstitut für Bau-, Stadt- und Raumforschung. Laufende Stadtbeobachtung - Raumabgrenzungen. Bonn: Bundesinstitut für Bau-, Stadt- und Raumforschung; 2015. Zugänglich unter/available from: http://www.bbsr.bund.de/BBSR/DE/ Raumbeobachtung/Raumabgrenzungen/StadtGemeindetyp/ StadtGemeindetyp_node.html
20. Bortz J, Doering N. Forschungsmethoden und Evaluation: Für Human- und Sozialwissenschaftler. 4 ed. Berlin, Heidelberg: Springer; 2006. DOI: 10.1007/978-3-540-33306-7

21. Verma P, Ford JA, Stuart A, Howe A, Everington S, Steel N. A systematic review of strategies to recruit and retain primary care doctors. BMC Health Serv Res. 2016;16(1):126. DOI: 10.1186/s12913-016-1370-1

22. Jungbauer J, Kamenik C, Alfermann D, Brähler E. Wie bewerten angehende Arzte rückblickend ihr Medizinstudium? Ergebnisse einer Absolventenbefragung. Gesundheitswesen. 2004;66(1):5156. DOI: $10.1055 / \mathrm{s}-2004-812705$

23. Bundesministerium für Bildung und Forschung. Masterplan Medizinstudium 2020. Berlin: Bundesministerium für Bildung und Forschung; 2017. Zugänglich unter/available from: https:/ /www.bmbf.de/files/2017-03-31_Masterplan\% 20Beschlusstext.pdf

\section{Korrespondenzadresse:}

Linda Barthen

Goethe-Universität Frankfurt, Institut für

Allgemeinmedizin, Theodor Stern Kai 7, 60590

Frankfurt/Main, Deutschland

barthen@allgemeinmedizin.uni-frankfurt.de

\section{Bitte zitieren als}

Barthen L, Ravens-Taeuber G, Paulitsch MA, Gerlach FM, Sennekamp M. How can General Practice be incorporated longitudinally in medical studies? Students' views on the development of a new rural health program. GMS J Med Educ. 2018;35(3):Doc42.

DOI: 10.3205/zma001188, URN: urn:nbn:de:0183-zma0011886

\section{Artikel online frei zugänglich unter}

http://www.egms.de/en/journals/zma/2018-35/zma001188.shtml

Eingereicht: 12.10 .2017

Überarbeitet: 17.05 .2018

Angenommen: 06.06.2018

Veröffentlicht: 15.08.2018

\section{Copyright}

(c)2018 Barthen et al. Dieser Artikel ist ein Open-Access-Artikel und steht unter den Lizenzbedingungen der Creative Commons Attribution 4.0 License (Namensnennung). Lizenz-Angaben siehe http://creativecommons.org/licenses/by/4.0/. 


\title{
"Landpartie 2.0" - Conceptual development and implementation of a longitudinal priority program to promote family medicine in rural areas
}

\begin{abstract}
Objective: This article reports on the conceptual development and subsequent implementation of a targeted and attractive general practice teaching program in a rural area for students of human medicine at the medical faculty of Goethe University, Frankfurt am Main.

Project description: Since the 2016/2017 winter semester, usually up to 15 interested students a year have had the opportunity to participate in the longitudinal priority program "Landpartie 2.0". The program runs for six semesters during the clinical stage of medical studies and consists of regular internships during which the students receive one-toone support in a family practice, and participate in a series of seminars and an annual one-day excursion. The aim is to enable students, early on in their studies and without any obligations, to gain uninterrupted experience of providing patient care, and to find out what it means to pursue a career in family medicine.

Results: Since the beginning of the annual program, 62 students have been included in it. The initial results show that the different elements of the program fulfil the expectations and requirements of participants and that their overall level of satisfaction is high. Almost $95 \%$ of students reported that they felt their knowledge had increased as a result of the internships, and they rated them as a useful part of their medical education. Despite the rural focus of the program, around half of the participants were of urban origin.

Conclusion: The "Landpartie 2.0" provides students with the opportunity to familiarize themselves with family health care in a rural area. Further studies should investigate to what extent the program encourages students to continue their training with a view to pursuing a career in family medicine.
\end{abstract}

Keywords: family medicine, medical students, curriculum, rural health program, shortage of family doctors

\section{Background}

It is possible to establish and reinforce an interest in general practice by strongly supporting the subject during medical studies [1], [2]. Priority programs in general practice can contribute towards ensuring that enough young doctors choose to enter the profession. This is particularly relevant in view of the shortage of new recruits that has been the subject of discussion for many years. Many such programs have been available outside Germany for decades [3], [4], [5], and a similar development has recently begun in Germany as well [6], [7], [8]. The results of preliminary evaluations have shown that participation in such a program can have a positive influence on preferences in favor of specialization in general practice [9] and possibly pursuing a career in the field [10]. Following this development, Goethe University in Frankfurt am Main has worked on a needs- and student-oriented priority program for the promotion of family health care in rural areas.

A multistage process was chosen in the planning phase, starting with an international literature search according to the snowball principle. Numerous and diverse programs were identified, most of which were in North America and Australia [3], [4], [5]. The accompanying analyses, which included long-term studies, demonstrated a positive effect on the choice of a later career as a rural physician [11]. However, entrance requirements for medical studies, as well as curricula and examination procedures, differed, sometimes considerably, from the German system, making it difficult to adopt them without changes. For this reason, we will focus in the following on existing developments and conditions in Germany.

In addition to the literature search, we contacted local experts that are interested in supporting young general practitioners in rural areas [12], [13], and discussed how 
to organize a priority program, and what should be the content of such a program.

The focus of most of the conceptual development phase was on the courses and overall conditions existing at German universities. As no systematic overview was available of the efforts that German universities were already making to strengthen general practice in rural areas, we conducted a survey of all medical faculties in Germany [6]. In addition, students at Goethe University Frankfurt were asked what they expected of such a priority program [14].

\section{Survey of all medical faculties in Germany}

Between July and November 2015, all 37 general practice teaching institutes in Germany were asked what programs they have, or are planning to introduce, to support family medicine in rural areas. The authors received feedback from 31 of the institutes (response rate $83.8 \%$ ). Twelve of the faculties had 13 relevant programs that focused on supporting family medicine during medical studies. Three of them lasted at least two semesters. Attributes shared by most of the programs were that the number of places available per year was limited and the programs had only recently been introduced. It was noteworthy that unlike the program we were planning, none of the identified priority programs explicitly focused on supporting family medicine in rural areas [6].

\section{Student survey at the medical faculty of Goethe University}

In order to help ensure the program was well accepted by the target group, we also asked students what they would want and require of a priority program by means of an online survey in summer 2015 [15]. Of the students, 617 in their fourth preclinical semester and later (response rate $28.7 \%$ ) took part. The results showed that interest in a possible program was considerable, whereby students attached most importance to a program that was practice-oriented, i.e. that included experiencing and actively working in a family practice. They also felt it was important to get to know what running a practice meant from an administrative and commercial perspective [14]. The online survey enabled us to gather information and suggestions relating to the organization and content of the individual elements of the program. For example, it was specified that the teaching course should start during the clinical stage of studies, and that besides the continuous practice orientation that is the central element of the program, the series of seminars should include subjects relating to running and managing a practice.

Using the described multistage development procedure, the priority program "Landpartie 2.0" ("rural outing 2.0") was developed on the basis of the "Landpartie $1.0^{\prime 1}$ that has existed since 2012. This paper presents the concept and preliminary evaluation results.

\section{Project description}

Based on the development stages presented above, the longitudinal six-semester (three-year) "Landpartie 2.0" teaching program was developed between April 2015 and September 2016.

The optional teaching program is directed at students in their first and, in exceptional circumstances, third clinical semesters. In order to apply, the students must complete a self-developed questionnaire and prepare a letter of motivation. As well as requiring socio-demographic details, and information on the careers the students currently foresee for themselves, the application documents also include questions on students' personal attitudes towards family medicine. Some of the questions are closed and some contain free-text fields, so that applicants have room to individualize their responses, while ensuring manageable comparisons remain possible. Completed questionnaires are then rated according to a fixed point system. The selection of participants takes place on the basis of predictors that correlate positively with a later decision to pursue a career in family medicine [16] and/or to work in a rural area [17], [18]. For example, the probability of being accepted in the program is higher when applicants say they have grown up in a rural region, and are particularly interested in developing long-term relationships to patients or treating a wide range of illnesses in their later day-to-day work. Overall, up to 15 students can generally be included in the program each year, meaning that in three annual cohorts, an average of 45 students can be taking part in the program at any one time. After the recruitment of the students, the program begins with a kick-off event that serves to introduce them to the content and organization of the program and enables all the participants to get to know one another. From the second clinical semester onwards. The practical part of "Landpartie 2.0" begins, which lasts until the end of the clinical phase of studies (see figure 1).

One of the most important criteria in the development of "Landpartie 2.0" was that the students' workload should only increase marginally. For this reason, existing compulsory courses and the time allocated to them were incorporated into a new organisational structure and were partly restructured contentwise. By participating in the program, the students complete the obligatory "General practice course" as well as the complete "Clinical elective" and the "Block internship", but simply in a new and restructured form. In addition, students have the free option to take advantage of organizational support and help in the procurement of clinical traineeships as well as placement in a general practice during the four-month elective in the practical year.

Regular practical experience with one-to-one support in rural family practices is at the core of the reorganization. The practices are located in one of the three participating districts - Bergstrasse, Hochtaunus and Fulda. Depending on semester, the students spend two to five days (per semester) at their allocated practice. Practice internships, on the other hand, always take place during semester 


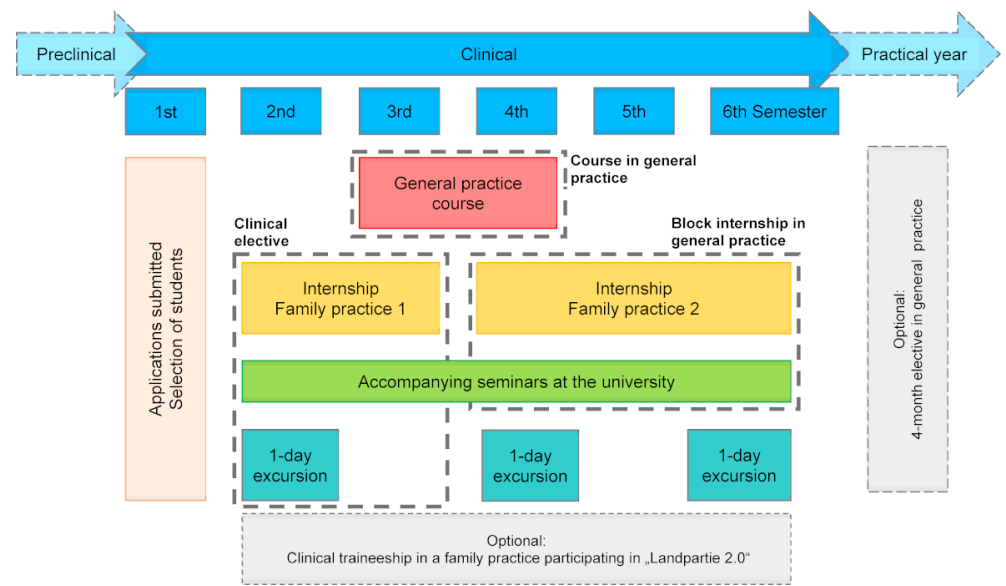

Figure 1: Curriculum timeline of "Landpartie 2.0" in clinical stage of medical studies.

breaks, with the exact period being decided upon by the students and the teaching physician.

Most of the teaching physicians were recruited especially for "Landpartie 2.0". When selecting the practices, we ensured that they not only fulfilled standard requirements for academic teaching practices, but were also located in rural communities ${ }^{2}$. After inclusion in the program, all the new teaching physicians are introduced to the organization of the program and undergo one-time training in didactics. Overall, it was possible to recruit 20 teaching physicians, practicing in communities with an average population of 10,238 [range: $2,430-25,345$, status as of 30.9.2019], for the "Landpartie 2.0" program.

In order to familiarize the students with the wide range of tasks that make up family health care, they get to know two practices during five semesters, of which one might be a single-handed practice in a community of 20,000 inhabitants and the other a joint practice in a community of 2,700 . The costs of travel and accommodation are covered by the participating districts, and the students receive a catering allowance.

Parallel to the practice internships, the Institute of General Practice provides an accompanying seminar, covering a wide range of topics ranging from case reviews, a personal exchange of information, strategies used to facilitate consultations (e.g. "breaking bad news") to the discussion of strategies related to specific therapies. Interdisciplinary subjects (e.g. "Outpatient Palliative Care" and "Psychosomatic Medicine for Outpatients"), as well as the commercial/organizational aspects involved in running a practice (e.g. "Opportunities in the Family Practice - Registration and Remuneration") are also covered in the series of seminars. The specific topics depend on students' needs and complement previous study content. Most of the lecturers come from the field of family medicine, with a few coming from the public health sciences. For participants in "Landpartie 2.0", the "General Practice Course" takes place in one of the teaching practices in the rural area and in particularly small groups with a maximum of seven students (generally 8-10). The duration of the course is 10.5 hours and takes place in either the third or fourth clinical semester. Amongst other topics, the students learn about typical procedures and disease patterns that they are likely to be confronted with in a family practice.

Once a year, participants in the program also have the opportunity to go on a one-day excursion to get to know innovative healthcare models. On this occasion, they are introduced to a broad spectrum of different approaches to organizing and collaborating in health care. The destinations of the excursions can be physician and health networks, joint practices, medical care centers and health centers. Participation in one excursion during the threeyear period is compulsory.

Furthermore, in order to support the students and teaching physicians, a logbook is provided, which serves as both a documentation aid throughout the whole program, as well as a basis for organizing the content of internships. The logbook provides the opportunity to stipulate learning objectives for individual internships and thus delivers an overview of the development of individual skills. The main areas of expertise include "Medical Interview Strategies", "Physical Examination", "The Use of Diagnostic Tools" and "Therapy/Prevention". The development of learning objectives is based to a significant extent on those that are used in the standard family medicine internship and that have been tried and tested during medical studies over the long term [19]. The logbook also contains feedback sheets, attendance sheets for all compulsory courses, and assessment sheets for grading students.

Besides reimbursement for travel and accommodation costs, the students also receive a book voucher and financial support for participation in scientific congresses linked to general practice.

\section{Results}

Using a number of methodological steps in its development, the priority program “Landpartie 2.0 " was successfully designed and implemented and embeds family medicine in the clinical phase of medical studies both longitudinally and with a practical orientation.

During the winter semester 2016/17, the "Landpartie 2.0" was offered to medical students for the first time. 
Applications were generally possible in the first clinical semester and the initial number of annual participants was 15. In the first three years, however, the number of applications from students in their first clinical semester was lower than the number of available places. Project management therefore decided to offer the program to students in their third clinical semester as well. However, as they were more advanced in their studies, they only completed the second phase of the internship in a family practice and only some of the program seminars. In this manner, it was possible to fill 16, 15 and 14 of the available places. In the winter semester of 2019/20, the number of applicants was 26 and thus greater than the number of available places for the first time. In response to the number of applicants and the capacity situation, it was therefore decided that two additional students should be allocated a place on the basis of the selection criteria. Since the winter semester 2016/17, it has thus been possible to include 62 students in the program.

Up to now, the participants have differed considerably from one another in terms of socio-demographic characteristics (see table 1$)$. The ratio of women (69.4\%) to men (30.6\%) reflects the situation in medical studies overall, but has nonetheless varied from year to year. The average age of participants at the time of application was 24 years (range 20-48), and, as expected, older applicants tended to already have completed vocational training of some kind. On the other hand, it was surprising that around half of the students had come from communities with more than 10,000 inhabitants and thus, according to the definition used in the program, had not grown up in a rural area. This result is in line with free-text responses provided on the application forms. While some applicants repeatedly expressed having a personal connection to the country, other students explicitly mentioned that despite having been raised in a large town, they wanted to take advantage of the opportunity to get to know a different lifestyle and another way of practicing family medicine.

The preliminary evaluation is of the time spent in the family practices, the accompanying seminars and of the annual one-day excursions, up to the end of the summer semester 2019. Over the long term, a graduate destination survey will also be used find out what actually influenced the decision of students to later work as a family practitioner (in a rural area), and their overall career paths.

All participants completed a two- to five-day internship in the family practices to which they were allocated once per semester, or twice a year. The students had to have attended each family practice at least twice before it was possible to switch. The evaluation then took place per practice and not per internship. Figure 2 shows the results from the first two years of the program. It shows that the level of satisfaction was very high with regard to the practical elements of the program. Almost 95\% of participants also reported that they felt their level of knowledge had increased and that they considered the practical internships to be a useful part of their medical edu- cation. Overall, the internships were allocated the school grade $1.51(n=37)$.

Two two-hour seminars take place per semester. The instructors and their number (between one and three instructors per seminar) vary depending on subject. Figure 2 shows that the demands that students had of the organization and content of the seminars were almost completely fulfilled, with $93 \%$ of students confirming that the content of the seminars was relevant and that their level of knowledge had risen as a result. The instructors were also unanimously considered to demonstrate high levels of motivation and to present the teaching material in a structured manner.

Since the beginning of "Landpartie 2.0", three one-day excursions to get to know different innovative models of healthcare have taken place in one of the participating districts. One excursion, for example, was to Ärztehaus Weilrod in Hochtaunuskreis. Six family practitioners work together in the healthcare center, which is made up of four single-handed practices and one group practice. Starting with a self-initiated quality circle that later developed into the organization of a local emergency service, the physicians now work closely with further specialist physicians in the region [20]. Excursion destinations such as Ärztehaus Weilrod provide the participants with an insight into a broad spectrum of physician and interdisciplinary cooperation models. The results of the evaluation show that students' expectations were consistently met (see figure 2). The organization also received their approval, with $91 \%$ of students saying the excursion had contributed towards increasing their level of knowledge, while $94 \%$ felt they had gained knowledge of different professional opportunities. Overall, $98 \%$ would recommend the excursion to others. The average school grade allocated to it was $1.43(n=47)$.

\section{Discussion}

Using a broad-based methodological approach, it was possible to conceptually develop the "Landpartie 2.0" priority program between 2015 and 2016 and to implement the changes from 2016/2017 onwards. Since the beginning of the teaching program, 62 interested students have been motivated to take part in it.

At its core, the "Landpartie 2.0" consists of regular internships at selected and trained rural practices in the Hessian districts of Bergstrasse, Fulda and Hochtaunuskreis. The internships are accompanied by seminars, the "General Practice Course" in small groups, and an annual one-day excursion to become acquainted with innovative heath care models. In order to prevent the students' workload from increasing, or to at least confine any increase to a minimum, the students that participated in the program were automatically considered to have completed the obligatory "Clinical elective" and "Block internship in General Practice".

As recommended by medical experts and students [21], [22], program participation leads to an increase in the 
Table 1: Socio-demographic details at time of application (around November of each year); (Source: proprietary research).

\begin{tabular}{|c|c|c|c|c|c|c|c|c|}
\hline & \multicolumn{2}{|l|}{ Semester } & \multicolumn{2}{|l|}{ Gender } & \multirow[b]{2}{*}{$\begin{array}{l}\text { Average age in } \\
\text { years [age range] }\end{array}$} & \multicolumn{2}{|c|}{ Origin (number) } & \multirow{2}{*}{$\begin{array}{l}\text { Completed vocational } \\
\text { training (number) }\end{array}$} \\
\hline & $\begin{array}{l}\text { first clinical } \\
\text { semester }\end{array}$ & $\begin{array}{l}\text { third clinical } \\
\text { semester }\end{array}$ & male & female & & $\begin{array}{l}\text { Rural } \\
\text { up to } 10,000 \\
\text { inhabitants }\end{array}$ & $\begin{array}{l}\text { Urban } \\
>10,000 \\
\text { inhabitants }\end{array}$ & \\
\hline $\begin{array}{l}\text { Cohort } \\
2016 / 17\end{array}$ & 11 & 5 & 7 & 9 & $\begin{array}{c}26 \\
{[20-48]}\end{array}$ & 6 & 10 & 4 \\
\hline $\begin{array}{l}\text { Cohort } \\
2017 / 18\end{array}$ & 12 & 3 & 3 & 12 & $\begin{array}{c}22 \\
{[20-25]}\end{array}$ & 4 & 11 & 1 \\
\hline \multirow{2}{*}{$\begin{array}{l}\text { Cohort } \\
2018 / 19\end{array}$} & \multirow[t]{2}{*}{13} & \multirow[t]{2}{*}{1} & \multirow{2}{*}{7} & \multirow{2}{*}{7} & \multirow{2}{*}{$\begin{array}{c}27 \\
{[21-37]}\end{array}$} & 8 & 5 & \multirow{2}{*}{9} \\
\hline & & & & & & \multicolumn{2}{|c|}{1 "don’t know" } & \\
\hline $\begin{array}{l}\text { Cohort } \\
2019 / 20\end{array}$ & 17 & 0 & 2 & 15 & $\begin{array}{c}23 \\
{[20-29]}\end{array}$ & 12 & 5 & 3 \\
\hline \multirow{2}{*}{ Overall } & 53 & 9 & 19 & 43 & \multirow{2}{*}{$\begin{array}{c}24 \\
{[20-48]}\end{array}$} & \multirow{2}{*}{30} & \multirow{2}{*}{31} & \multirow{2}{*}{17} \\
\hline & & & & & & & & \\
\hline
\end{tabular}

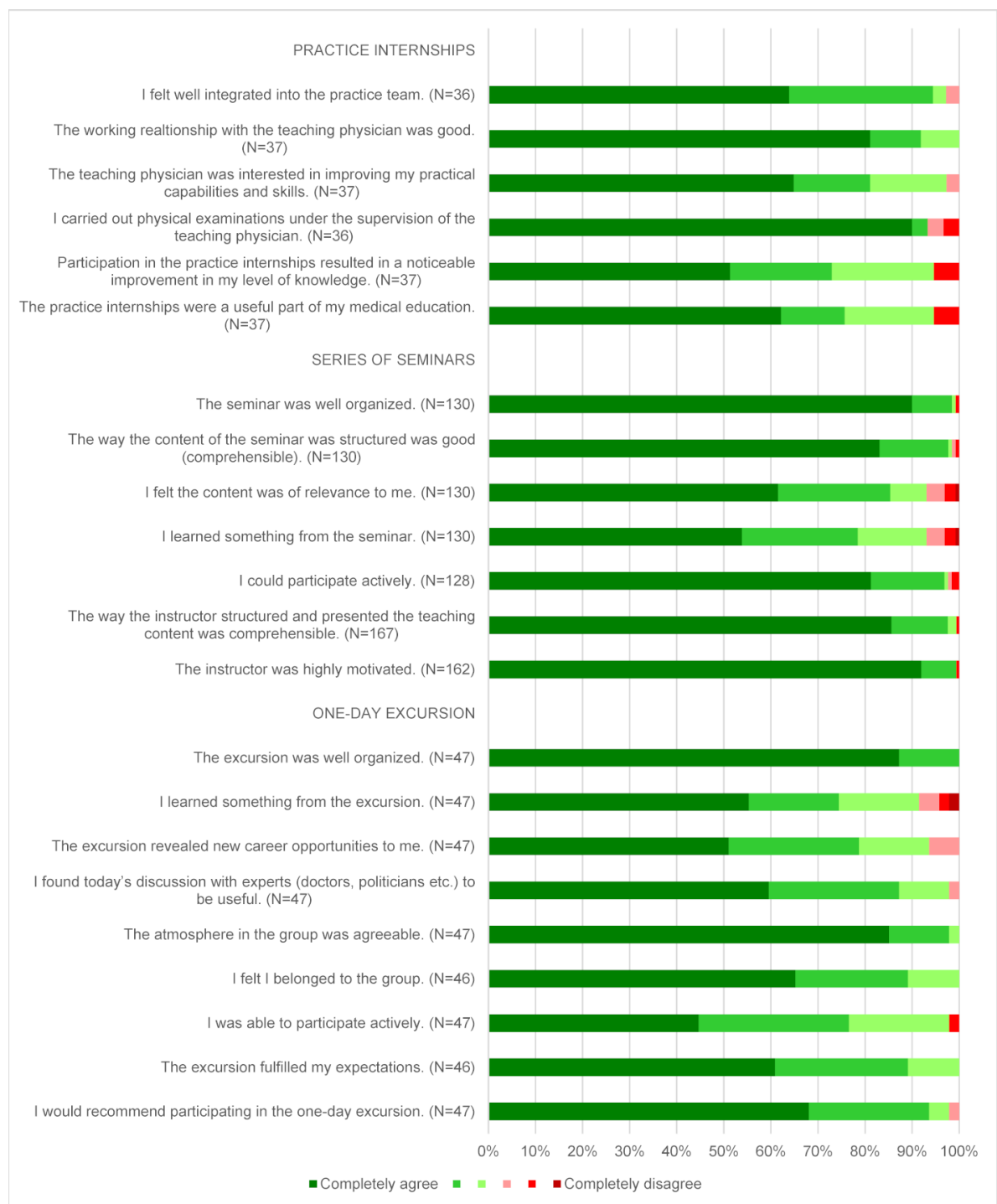

Figure 2: Evaluation results of the practice internships, series of seminars and the one-day excursion up to the summer semester 2019 (Source: proprietary presentation)

practical share of students' education in general practice. The objective is that by experiencing intense contact with patients early on, participants should develop and strengthen their practical, communication and social skills. The academic teaching physicians serve the students as role models and mentors, both in seminars and in the practices, and are thus able to strengthen student interest in family medicine [23].

The voluntary character of the program enables participants to get to know both family health care and what it means to work in a rural area without having to commit themselves over the long term, as they would if they set 
up in private practice in one of the three participating districts. Recurring and early contact with family health care and medical care in rural areas can also have an "adhesive effect". The evaluation of similar programs has demonstrated a positive effect on readiness to work as a family practitioner (in a rural area) after completing medical studies [11].

The "Landpartie 2.0" program can thus be considered an alternative to the "rural physician quotas" that have recently been introduced by several federal German states. Whereas the "rural physician quota" represents a binding decision to choose to work as a family doctor in a rural area previous to medical studies [24], the priority program described here provides students with the opportunity to try working as a young doctor and to get to know their own personal interests, based on the appeal of working in a rural area, and their own intrinsic motivation. At the same time, they can develop their medical skills and competencies.

Preliminary evaluation results show that the expectations and demands that students wished to see fulfilled by "Landpartie 2.0" were satisfied, and approval of practice internships, the series of seminars and the annual oneday excursion was high to very high. Over the mediumand long-term, evaluative graduate destination surveys will illustrate whether participation in the program increases interest in family medicine. They will also show whether, in comparison to students with similar interests that did not participate in the program, the probability is higher that participants will choose to do a four-month elective in their practical year and/or specialist training in family medicine, and whether they will later decide to work as family doctors (in a rural region).

The present evaluation takes its place among further investigations that have been conducted into other established programs that are available in Germany. Since 2011, the "Family Medicine Class" has provided 20 medical students with a clinical elective that focuses on primary care. An evaluation after the first two years showed that the acquisition of skills resulting from participation in the program had a positive influence on the decision to select family medicine as a medical specialty [9]. The study of a preclinical elective that focused on family healthcare in a rural area around Leipzig demonstrated that participation in a course on family medicine strengthened the position of family medicine as the first choice among career options [10].

Despite the comprehensive methodological approach employed in the development phase, it is by no means certain that the program developed for "Landpartie 2.0" will awaken the interest of medical students in the future. The question to what extent participation in the program increases the readiness to, for example, complete a fourmonth elective in the practical year and/or specialize in family medicine with a view to working in a rural area remains uncertain. Amongst other considerations, it should be taken into account that the participating students made up, a priori, a strongly self-selected group. Only students with a basic interest in family medicine, and who are at least prepared to consider specializing in the field and working in a practice outside a large city, are likely to apply to participate in the program. However, other similar programs also face this methodological problem [7], [25]. It is therefore essential to take this selection effect into account in more detailed evaluations. To the best of our knowledge, the longitudinal priority program presented here is the first in Germany to focus on promoting family medicine in a rural area during medical studies. Similar programs in other regions generally concentrate on family medicine but have no explicit rural focus. The broad methodological approach to the conceptual development of the program enabled best practices, the needs and wishes of students, as well as existing curricular and legal framework conditions in Germany to be taken into account early on in the process. In the development phase, it was therefore clear early on what long-term personnel and financial resources the program would have to mobilize to ensure its implementation was sustainable. Furthermore, the program was designed to be voluntary, available from the first clinical semester onwards, and to focus on practical training as far as possible.

\section{Conclusion}

The optional "Landpartie 2.0" program was successfully planned and integrated into the clinical phase of medical studies. The chosen methodological approach contributed towards ensuring that the opportunities and limitations of such a program were recognized and considered in its conceptual development.

Preliminary evaluation results indicate that "Landpartie 2.0" meets the needs of students, makes an important contribution towards improving medical training and, despite its rural focus, also appeals to students from more urban regions. Overall, it is the view of the authors that the longitudinal and comprehensive character of the developed priority program can positively influence the further training and career paths chosen by medical students in favor of family medicine. Participation in a voluntary teaching program that forms an integral part of medical studies is therefore a promising alternative to the so-called "rural physician quota".

However, any attempt to secure the availability of young professionals in a specific field requires corresponding personnel and financial resources, and these are not (currently) available at a number of locations. The repeatedly affirmed readiness to promote family medicine in medical studies should therefore be followed by concrete steps, and should in particular be aimed at ensuring the sustainable financial footing required to implement the necessary measures. 


\section{Notes}

${ }^{1}$ The "Landpartie 1.0" permits interested students to complete a block internship in family medicine in rural practices in cooperation with the District of Fulda. It is complemented by special undertakings and offers (Event Day, voucher for leisure activities, reimbursement of costs of accommodation and travel)

${ }^{2}$ Our intention was that practices should be in communities with fewer than 20,000 inhabitants. With two exceptions (both with 25,000 inhabitants), it was possible to satisfy this criterion.

\section{Acknowledgements}

We would like to thank all the practices, districts, experts and students that played such an active role in helping us develop "Landpartie 2.0".

\section{Funding}

This project was supported by local authorities in the districts of Bergstrasse, Fulda and Hochtaunuskreis.

\section{Competing interests}

The authors declare that they have no competing interests.

\section{References}

1. Deutsch T, Lippmann S, Sandholzer H. Gewinnung hausärztlichen Nachwuchses-Zusammenhang zwischen praxisorientierter Lehre und Karriereentscheidung. Gesundheitswesen. 2014;76(01):2631. DOI: $10.1055 / \mathrm{s}-0033-1334933$

2. Nicholson S, Hastings AM, McKinley RK. Influences on students' career decisions concerning general practice: a focus group study. Br J Gen Pract..2016;66(651):e768-775. DOI: 10.3399/bjgp16X687049

3. Krupa LK, Chan BT. Canadian rural family medicine training programs: growth and variation in recruitment. Can Fam Physician. 2005;51:852-853.

4. Eley DS, Synnott R, Baker PG, Chater AB. A decade of Australian rural clinical school graduates - where are they and why? Rural Remote Health. 2012;12:1937. Zugänglich unter/available from: http://www.rrh.org.au/publishedarticles/article_print_1937.pdf

5. Rabinowitz HK, Diamond JJ, Markham FW, Wortman JR. Medical school programs to increase the rural physician supply: a systematic review and projected impact of widespread replication. Acad Med. 2008;83(3):235-243. DOI: 10.1097/ACM.0b013e318163789b

6. Barthen L, Beig I, Sennekamp M, Gerlach FM, Erler A, RavensTaueber G. Raus aufs Land während des Medizinstudiums: Eine Übersicht zu bestehenden und geplanten allgemeinmedizinischen Förderangeboten. Z Allg Med. 2016;92(11):448-445. DOI: 10.3238/zfa.2016.0448-0455
7. Blozik E, Ehrhardt M, Scherer M. Förderung des allgemeinmedizinischen Nachwuchses: Initiativen in der universitären Ausbildung von Medizinstudierenden. Bundesgesundhbl Gesundheitsforsch Gesundheitsschutz. 2014;57(7):892-902. DOI: 10.1007/s00103-014-1984-6

8. Schäfer H, Mangold B, Paulitsch M, Gerlach FM. Landpartie Fulda - ein Projekt zur Förderung des hausärztlichen Nachwuchses im ländlichen Raum. Z Allg Med. 2015;91(6):260-263. DOI: 10.3238/zfa.2015.0260-0263

9. Samos FA, Heise M, Fuchs S, Mittmann S, Bauer A, Klement A. A pilot phase evaluation of the elective general pracitice class: results of student surveys of the first two years. GMS J Med Educ. 2017;34(1):Doc4. DOI: 10.3205/zma001081

10. Deutsch T, Hönigschmid P, Frese T, Sandholzer H. Early community-based family practice elective positively influences medical students' career considerations - a pre-post-comparison. BMC Fam Pract. 2013;14:24. DOI: 10.1186/1471-2296-14-24

11. Goodfellow A, Ulloa JG, Dowling PT, Talamantes E, Chheda S, Bone C, Moreno G. Predictors of primary care physician practice location in underserved urban or rural areas in the United States: A systematic literature review. Acad Med. 2016;91(9):13131321. DOI: $10.1097 /$ ACM.0000000000001203

12. Bayerisches Ärzteblatt. Hausarztleben im Bayerischen Wald. Bay Ärztebl. 2016. Zugänglich unter/available from: https:// www.bayerisches-aerzteblatt.de/inhalte/details/news/detail/ News/hausarztleben-im-bayerischen-wald.html

13. Nohl-Deryk P. Landarzt-Famulaturen: Heiss begehrt. Ärztebl. 2015. Zugänglich unter/available from: https:// www.aerzteblatt.de/archiv/172217/Landarzt-Famulaturen-Heissbegehrt

14. Barthen L, Ravens-Taeuber G, Paulitsch MA, Gerlach FM, Sennekamp M. How can General Practice be incorporated longitudinally in medical studies? Students' views on the development of a new rural health program. GMS J Med Educ. 2018;35(3):Doc42. DOI: 10.3205/zma001188

15. Yengo-Kahn AM, Baker CE, Lomis KD. Medical students' perspectives on implementing curriculum change at one institution. Acad Med. 2017;92(4):455-461. DOI: 10.1097/ACM.0000000000001569

16. Deutsch T, Lippmann S, Frese T, Sandholzer H. Who wants to become a general practitioner? Student and curriculum factors associated with choosing a GP career - a multivariable analysis with particular consideration of practice-orientated GP courses. Scand J Prim Health Care. 2015;33(1):47-53. DOI: 10.3109/02813432.2015.1020661

17. Puddey I, Mercer A, Playford, D, Pougnault S, Riley G. Medical student selection criteria as predictors of intended rural practice following graduation. BMC Med Eeduc. 2014;14(1):218. DOI: 10.1186/1472-6920-14-218

18. Viscomi M, Larkins S, Gupta TS. Recruitment and retention of general practitioners in rural Canada and Australia: a review of the literature. Can J Rural Med. 2013;18(1):13-23.

19. Ravens-Taeuber G, Wunder A, Güthlin C, Koné I. Checkliste Blockpraktikum Allgemeinmedizin. Wie beeinflusst die systematische Festlegung von ausgewählten Lernzielen den subjektiven Lernfortschritt der Studierenden? Z Allg Med. 2019;95(7):448-445. DOI: 0.3238/zfa.2019.0307-0313

20. Ärztehaus Weilrod. Innovative Gesundheitsmodelle. Weilrod: Ärztehaus Weilrod. Zugänglich unter/available from:http:// www.innovative-gesundheitsmodelle.de/modelle/-/asset_ publisher/ubf7syrf6E2N/content/arztehaus-weilrod/maximized 
21. Bundesvertretung der Medizinstudierenden in Deutschland (bvmd). Longitudinale Einbindung der Allgemeinmedizin in das Medizinstudium. Berlin: bvmd; 2014. Zugänglich unter/available from: https://www.bvmd.de/fileadmin/redaktion/ Positionspapiere/Positionspapier_2014-04-26_Longit.Einbindung-der-Allgemeinmed-in-Medizinstudium.pdf

22. Huenges B, Gulich M, Böhme K, Fehr F, Streitlein-Böhme I, Rüttermann V, Baum E, Niebling WB, Rusche $H$.

Recommendations for undergraduate training in the primary care sector - position paper of the GMA-Primary Care Committee. GMS Z Med Ausbild. 2014;31(3):Doc35. DOI: 10.3205/zma000927

23. Stagg P, Prideaux D, Greenhill J, Sweet L. Are medical students influenced by preceptors in making career choices, and if so how? A systematic review. Rural Remote Health. 2012;12:1832.

24. Bundesministerium für Bildung und Forschung. Masterplan Medizinstudium 2020. Berlin: Bundesministerium für Bildung und Forschung; 2017. Zugänglich unter/available from: https:/ /www.bmbf.de/files/2017-03-31_Masterplan\% 20Beschlusstext.pdf

25. Verma P, Ford JA, Stuart A, Howe A, Everington S, Steel N. A systematic review of strategies to recruit and retain primary care doctors. BMC Health Serv Res. 2016;16:126. DOI:

10.1186/s12913-016-1370-1

\section{Corresponding author:}

Linda Seeger, M.Sc. Public Health

Goethe University Frankfurt am Main, Institute of General Practice, Theodor-Stern-Kai 7, D-60590 Frankfurt/Main, Germany

seeger@allgemeinmedizin.uni-frankfurt.de

\section{Please cite as}

Seeger L, Becker N, Ravens-Taeuber G, Sennekamp M, Gerlach FM. "Landpartie 2.0" - Conceptual development and implementation of a longitudinal priority program to promote family medicine in rural areas. GMS J Med Educ. 2020;37(3):Doc29.

DOI: 10.3205/zma001322, URN: urn:nbn:de:0183-zma0013225

\section{This article is freely available from}

https://www.egms.de/en/journals/zma/2020-37/zma001322.shtml

Received: 2019-10-22

Revised: 2020-01-15

Accepted: 2020-02-11

Published: 2020-04-15

\section{Copyright}

(C)2020 Seeger et al. This is an Open Access article distributed under the terms of the Creative Commons Attribution 4.0 License. See license information at http://creativecommons.org/licenses/by/4.0/. 


\section{"Landpartie 2.0" - Konzeptionelle Entwicklung und Implementierung eines longitudinalen Schwerpunktprogramms zur Förderung der Allgemeinmedizin im ländlichen Raum}

\section{Zusammenfassung}

Zielsetzung: Dieser Beitrag berichtet über die konzeptionelle Entwicklung und anschließende Implementierung eines zielgruppenspezifischen und attraktiven allgemeinmedizinischen Lehrangebots im ländlichen Raum für Studierende der Humanmedizin am Fachbereich Medizin der Goethe-Universität, Frankfurt am Main.

Methodik: Seit dem Wintersemester 2016/2017 können in der Regel bis zu 15 interessierte Studierende pro Jahr an dem longitudinalen Schwerpunktprogramm der „Landpartie 2.0“ teilnehmen. Das für den klinischen Studienabschnitt konzipierte, longitudinal über sechs Semester reichende Angebot besteht aus regelmäßigen Praxisphasen in 1:1Betreuung, einer Seminarreihe und einem jährlichen Tagesausflug. Ziel ist das frühzeitige und kontinuierliche Erleben der Patientenversorgung, um so die eigenen Kompetenzen zu stärken und einen möglichen allgemeinmedizinischen Karriereweg anzuregen.

Ergebnisse: Seit dem Start des jährlichen Angebots konnten 62 Studierende in das Programm aufgenommen werden. Erste Evaluationsergebnisse zeigen, dass die Angebotsbestandteile die Erwartungen und Anforderungen der Teilnehmenden erfüllen und eine hohe Programmzufriedenheit besteht. Knapp 95 \% der Studierenden berichten von einem subjektiven Wissenszuwachs durch die Praxisphasen und stufen diese als nützlichen Abschnitt ihrer ärztlichen Ausbildung ein. Trotz des ländlichen Fokus des Programms, stammt etwa die Hälfte aller Teilnehmenden aus urbaneren Regionen.

Schlussfolgerung: Die „Landpartie 2.0“ stellt eine Möglichkeit dar, Studierende frühzeitig mit der hausärztlichen Versorgung im ländlichen Raum in Kontakt zu bringen. Inwieweit auf diese Weise der weitere Ausbildungs- und Berufsweg mit dem Ziel einer hausärztlichen Tätigkeit positiv beeinflusst wird, soll in Verbleibstudien evaluiert werden.

Schlüsselwörter: Allgemeinmedizin, Medizinstudierende, Curriculum, Landarztprogramm, Hausarztmangel

\section{Hintergrund}

Eine intensivere Förderung der Allgemeinmedizin bereits im Studium stellt eine Möglichkeit dar, ein grundlegendes Interesse am Fach zu etablieren oder zu bestärken [1], [2]. Bezugnehmend auf den seit Jahren diskutierten Nachwuchsmangel in der hausärztlichen Versorgung können allgemeinmedizinische Schwerpunktprogramme einen Beitrag zur fachspezifischen Nachwuchssicherung leisten. Während im Ausland bereits vielfältige Angebote seit Jahrzehnten bestehen [3], [4], [5], ist eine ähnliche Entwicklung zuletzt auch für Deutschland zu verzeichnen [6], [7], [8]. Erste Evaluationsergebnisse zeigen hierbei, dass der Kompetenzerwerb durch eine Programmteilnahme einen positiven Einfluss auf die Präferenz zur Fachgebietswahl Allgemeinmedizin hat [9] bzw. das Fach als erste Karriereoption bestärkt [10].

Dieser Entwicklung entsprechend sollte auch an der Goethe-Universität in Frankfurt am Main ein bedarfsgerechtes und studierendenorientiertes Schwerpunktprogramm zur Förderung der Allgemeinmedizin im ländlichen Raum entwickelt werden.

Für die Konzeption wurde auf ein mehrstufiges Verfahren zurückgegriffen. Begonnen wurde mit einer internationaIen Literaturrecherche nach dem Schneeballprinzip. Es konnten diverse Programme identifiziert werden, die jedoch vorrangig in Nordamerika und Australien etabliert sind [3], [4], [5]. Die begleitenden Evaluationen weisen 
u.a. durch langjährige Verbleibstudien auch hier einen positiven Effekt auf eine spätere Landarzttätigkeit nach [11]. Die Zugangsbedingungen zum Medizinstudium sowie die Curricula und Prüfungsformate unterscheiden sich jedoch teils erheblich vom deutschen System, wodurch eine Adaption nicht uneingeschränkt möglich ist. Aus diesem Grund wurde im weiteren Verlauf das Augenmerk auf die Entwicklungen und den Ist-Stand in Deutschland gelegt.

Neben der Literaturrecherche erfolgte so die Kontaktaufnahme zu hiesigen Experten, die sich um die Förderung des allgemeinmedizinischen Nachwuchses in ländlichen Regionen bemühen [12], [13]. Ziel des gemeinsamen Austauschs war die Gewinnung von projektrelevanten Informationen zur organisatorischen und inhaltlichen Entwicklung eines Schwerpunktprogramms.

Im Hauptteil der konzeptionellen Entwicklung wurde der Fokus auf Angebote und Rahmenbedingungen, die an Universitäten in Deutschland existieren, gelegt. Da eine systematische Übersicht fehlte, die darstellt, welche Angebote zur Stärkung der Allgemeinmedizin (im ländlichen Raum) an deutschen Universitäten bereits existieren, wurde eine Befragung aller medizinischen Fakultäten Deutschlands durchgeführt [6]. Zudem wurden Studierende der Goethe-Universität Frankfurt zu ihren Anforderungen an ein Schwerpunktprogramm befragt [14].

\section{Befragung aller medizinischen Fakultäten in Deutschland}

Von Juli bis November 2015 wurden alle 37 allgemeinmedizinischen Lehreinrichtungen in Deutschland befragt, welche Programme zur Förderung der Allgemeinmedizin auf dem Land vor Ort bestehen oder derzeit in Planung sind. Von 31 Einrichtungen erhielten die Autoren eine Rückmeldung (Rücklaufquote=83,8\%). An 12 Fakultäten wurden dabei 13 relevante Angebote identifiziert, die sich auf die Förderung der Allgemeinmedizin im Studium fokussieren. Drei davon sind auf eine Laufzeit von mindestens zwei Semestern angelegt. Gemeinsam ist den meisten Angeboten, dass die Zahl der Plätze pro Jahrgang begrenzt ist und die Angebote erst in den vergangenen Jahren entstanden sind. Auffallend war hingegen, dass keines der identifizierten Schwerpunktprogramme explizit damit wirbt, die Allgemeinmedizin im ländlichen Raum zu fördern, so wie es das Ziel der eigenen konzeptionellen Entwicklung war [6].

\section{Studierendenbefragung an der medizinischen Fakultät der Goethe-Universität}

Neben den Erfahrungen aus etablierten Angeboten sollten insbesondere die Vorstellungen der Zielgruppe in die Entwicklung einfließen, da dies zu einer erhöhten Akzeptanz bei der selbigen beitragen kann [15]. Dementsprechend wurde mittels einer Online-Befragung im Sommer 2015 erfasst, welche Wünsche und Anforderungen Medizinstudierende der Goethe-Universität an ein Schwerpunktprogramm haben. An der Befragung nahmen 617 Studie- rende ab dem 4. vorklinischen Semester teil (Rücklaufquote=28,7\%). Im Ergebnis zeigte sich, dass das Interesse an einem potentiellen Angebot groß ist. Dabei erwarten die Studierenden in erster Linie einen starken Praxisbezug - also das Erleben und die aktive Mitarbeit in Hausarztpraxen. Zudem gehören aus Sicht der angehenden Mediziner zu einem solchen Angebot auch das Kennenlernen administrativer sowie wirtschaftlicher Hintergründe zum Führen einer Praxis [14].

Durch die Online-Befragung konnten Hinweise und Anregungen für die organisatorische sowie inhaltliche Gestaltung der einzelnen Bestandteile des Programms gewonnen werden. So wurde z. B. definiert, dass das Lehrangebot erst ab dem klinischen Studienabschnitt startet und neben dem zentralen Element eines kontinuierlichen Praxisbezugs auch Themen zur Praxisführung und -management Bestandteile in einer begleitenden Seminarreihe sein sollten.

Auf Basis des beschriebenen, mehrstufigen Entwicklungsverfahrens wurde letztlich das Schwerpunktprogramm „Land partie 2.0“ entwickelt, das an der bereits seit 2012 bestehenden „Landpartie 1.0“" anknüpft. Dieser Beitrag stellt das Konzept und erste Evaluationsergebnisse vor.

\section{Projektbeschreibung}

Auf Basis der dargestellten Entwicklungsschritte wurde von April 2015 bis September 2016 das longitudinale, über sechs Semester (drei Jahre) reichende, Lehrangebot der „Landpartie 2.0“ konzipiert.

Das fakultative Lehrangebot richtet sich an Studierende des ersten klinischen Semesters. Diese können sich mittels eines eigens entwickelten Fragebogens/Motivationsschreibens bewerben. Der Bewerbungsbogen umfasst sozio-demografische Angaben, Angaben zum aktuell geplanten Karriereweg, aber auch Fragen zur persönlichen Einstellung gegenüber der Allgemeinmedizin. Es gibt dabei sowohl geschlossene als auch offene Fragen, um den Bewerber*innen möglichst viel Raum für individuelle Antworten zu lassen, aber gleichzeitig eine handhabbare Vergleichbarkeit zu ermöglichen. Der eingegangene Fragebogen wird anhand eines festgelegten Punkteschemas bewertet. Die Auswahl der Teilnehmenden erfolgt dabei auf Grundlage von Prädiktoren, die in einem positiven Zusammenhang mit einer späteren Karriereentscheidung der Studierenden in der Allgemeinmedizin [16] und/oder einer Tätigkeit im ländlichen Raum stehen [17], [18]. So erhöht sich beispielweise die Chance, in das Programm aufgenommen zu werden, wenn die Bewerber*innen angeben, selbst in einer ländlichen Region aufgewachsen zu sein, in ihrem späteren beruflichen Alltag insbesondere an einer Langzeitbeziehung zu Patient*innen oder einem breiten Krankheitsspektrum interessiert zu sein. Insgesamt können pro Jahrgang in der Regel bis zu 15 Studierende in das Programm aufgenommen werden. In drei Jahrgangs-Kohorten nehmen somit durchschnittlich 45 Studierende zeitgleich teil. Das Angebot startet nach der Rekrutierung der Studierenden mit einer Auftaktveranstal- 


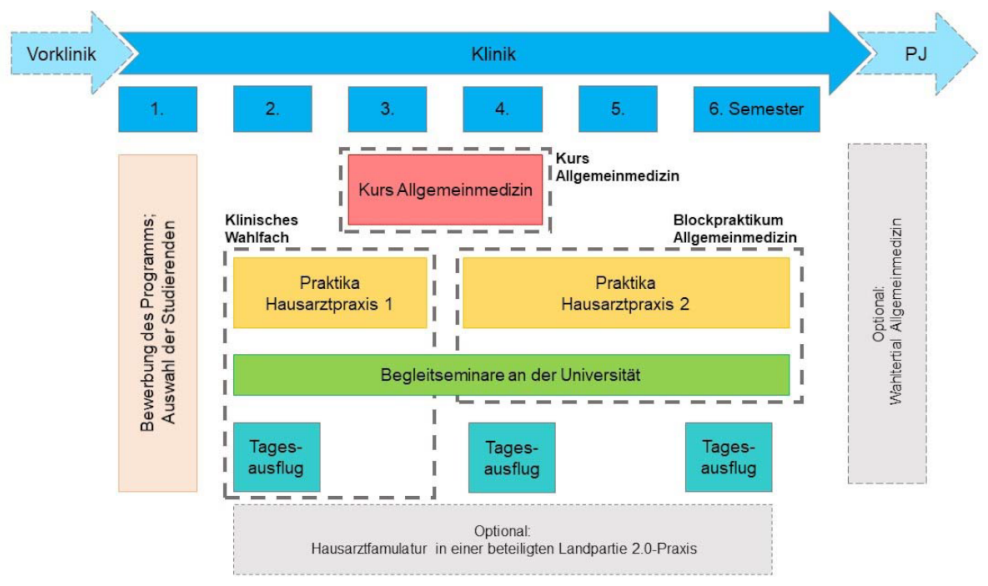

Abbildung 1: Curricularer Ablauf der "Landpartie 2.0" im klinischen Studienabschnitt

tung, die zur inhaltlichen und organisatorischen Einführung dient und ein erstes Kennenlernen zwischen allen Beteiligten ermöglicht. Ab dem zweiten klinischen Semester beginnt der praktische Teil der „Landpartie 2.0“, der sich insgesamt bis zum Ende des klinischen Studienabschnitts erstreckt (siehe Abbildung 1).

Bei der Entwicklung der „Landpartie 2.0“ war eines der wichtigsten Kriterien, dass für die Studierenden kein oder nur ein sehr geringer zeitlicher Mehraufwand entsteht. Aus diesem Grund wurden bereits bestehende curriculare Pflichtlehrveranstaltungen bzw. deren festgesetztes Zeitkontingent aufgegriffen und organisatorisch sowie zum Teil inhaltlich neu strukturiert. Im Ergebnis absolvieren die Studierenden somit durch ihre Teilnahme am Programm den obligatorischen „Kurs Allgemeinmedizin“, das gesamte „klinische Wahlfach“ sowie das „Blockpraktikum Allgemeinmedizin“ - aber in einem neuen, strukturierten Design. Optional können die Studierenden auch bei der Organisation und Vermittlung von Famulaturplätzen sowie dem Wahltertial im Praktischen Jahr in der Allgemeinmedizin unterstützt werden.

Im Zentrum der Neuausrichtung stehen regelmäßige praktische Erfahrungen in 1:1-Betreuung in ländlich gelegenen Hausarztpraxen. Diese befinden sich in einem der drei teilnehmenden Landkreise - Bergstraße, Hochtaunus oder Fulda. Die Studierenden gehen pro Semester für zwei bis fünf Tage (in Abhängigkeit des jeweiligen Semesters) in ihre zugewiesene Praxis. Die Praxisphasen finden stets in der vorlesungsfreien Zeit statt und der konkrete Termin wird individuell zwischen Studierenden und Lehrärzt*innen geplant.

Die Mehrheit der Lehrärzt*innen wurde dabei eigens für die „Landpartie 2.0“ neu rekrutiert. Bei der Auswahl der Praxen wurde darauf geachtet, dass sie nicht nur die regulären Aufnahmebedingungen für akademische Lehrpraxen erfüllen, sondern auch in ländlich gelegenen Kommunen liegen ${ }^{2}$. Nach der Aufnahme in das Programm durchlaufen alle neuen Lehrärzt*innen eine organisatorische Einführung und eine einmalige Didaktikschulung. Insgesamt konnten bisher 20 Lehrärzt*innen, die im Durchschnitt in Gemeinden mit einer Einwohnerzahl von 10.238 praktizieren [Range: 2.430-25.345, Stand:
30.9.2019], in das Programm der „Landpartie 2.0“ aufgenommen werden.

Um ein breites Spektrum hausärztlicher Versorgung zu erleben, lernen die Studierenden innerhalb von fünf Semestern zwei verschiedene Praxen kennen (z. B. Praxis 1: Einzelpraxis in einer Kommune mit 20.000 Einwohner*innen; Praxis 2: Gemeinschaftspraxis in einer Kommune mit 2.700 Einwohner*innen). Um einen finanziellen Aufwand für die teilnehmenden Studierenden zu vermeiden, werden Fahrt- und Übernachtungskosten sowie eine Verpflegungspauschale durch die beteiligten Landkreise übernommen.

Die Praxisphasen werden von einem begleitenden Seminar am Institut für Allgemeinmedizin umrahmt. Das Themenspektrum ist vielfältig und reicht von Fallbesprechungen, persönlichem Erfahrungsaustausch, Strategien zur Gesprächsführung (z.B. Thema „Breaking Bad News“) bis hin zur Besprechung von Therapiestrategien. Aber auch interdisziplinäre Themen (z.B. „ambulante Palliativmedizin“ oder „ambulante Psychosomatik“) sowie wirtschaftliche/organisatorische Aspekte zum Führen einer Praxis (z.B. „Möglichkeiten in der hausärztlichen Praxis - Niederlassung \& Honorar") sind Gegenstand der Seminarreihe. Die konkrete Themenfestsetzung hängt auch von dem Bedarf der Studierenden ab und stellt eine Ergänzung zu den bisherigen Studieninhalten dar. Die Dozent*innen stammen dabei vorrangig aus der Allgemeinmedizin aber vereinzelt auch aus den Gesundheitswissenschaften.

Der „Kurs Allgemeinmedizin“ erfolgt für die Teilnehmenden der „Landpartie 2.0“ in besonders kleinen Gruppen von max. sieben Studierenden (regulär 8-10 Studierende) in einer geschulten Hausarztpraxis auf dem Land. Der Kurs umfasst 10,5 Zeitstunden und findet im 3. oder 4. klinischen Semester statt. Die Studierenden lernen u. a. typische allgemeinmedizinische Vorgehensweisen und Krankheitsbilder der hausärztlichen Praxis kennen.

Zusätzlich wird den Teilnehmenden im Programm einmal pro Jahr ein Tagesausflug zu innovativen Modellen der Gesundheitsversorgung angeboten. Ziel ist es, das breite Spektrum an Kooperations- und Organisationsformen in der hausärztlichen Versorgung aufzuzeigen. Ausflugsziele können neben Arzt- oder Gesundheitsnetzen auch Gemeinschaftspraxen, medizinische Versorgungszentren 
oder Gesundheitszentren sein. Die Teilnahme an einem Tagesausflug innerhalb von drei Jahren ist dabei verpflichtend.

Zur Unterstützung der Studierenden und der Lehrärzt*innen wurde zudem ein eigenes Logbuch erstellt, das über das gesamte Programm hinweg als Dokumentationshilfe und als Grundlage für die inhaltliche Gestaltung der Praktika dient. Das Logbuch bietet die Möglichkeit, Lernziele für die einzelnen Praktika festzuhalten und somit auch einen Überblick über die individuelle Kompetenzentwicklung zu erstellen. Hauptkompetenzfelder sind dabei „Gesprächsführung“, „Körperliche Untersuchung“, „Gerätediagnostik“ sowie „Therapie/Prävention“. Die Entwicklung der Lernziele erfolgte in starker Anlehnung an denen, die bereits für das Blockpraktikum Allgemeinmedizin im regulären Studienverlauf entwickelt und langfristig erprobt wurden [19]. Ferner beinhaltet das Logbuch Feedbackbögen, Teilnahmebestätigungen für alle curricular verpflichtenden Veranstaltungen sowie die Bewertungsbögen für die Notenvergabe.

Neben der Erstattung von Fahrt- und Übernachtungskosten erhalten die Studierenden zudem pro Semester einen Büchergutschein und finanzielle Unterstützung bei der Teilnahme an wissenschaftlichen Kongressen mit allgemeinmedizinischem Bezug.

\section{Ergebnisse}

Mittels verschiedener methodischer Entwicklungsschritte konnte das Schwerpunktprogramm, „Landpartie 2.0“, das die Allgemeinmedizin im klinischen Studienabschnitt longitudinal und praxisnah verankert, konzeptioniert und erfolgreich implementiert werden.

Zum Wintersemester 2016/2017 wurde die Landpartie 2.0 den Medizinstudierenden erstmals angeboten. Bewerbungen waren normalerweise im ersten klinischen Semester möglich und die ursprüngliche Teilnehmerzahl pro Jahr betrug 15. In den ersten drei Jahren gingen jedoch weniger Bewerbungen aus dem ersten klinischen Semester ein als Plätze vorhanden waren. Aus diesem Grund entschied sich die Projektleitung dazu, das Programm auch den Studierenden im dritten klinischen Semester anzubieten. Da diese jedoch bereits weiter in ihrem Studium vorangeschritten sind, wird nur noch die Hausarztpraxisphase 2 und ein Teil der Seminare im Rahmen des Programms absolviert. Auf diese Weise konnten in den ersten drei Jahren 16, 15 und 14 Plätze belegt werden. Im Wintersemester 2019/2020 lag die Bewerberzahl mit 26 erstmals über den zur Verfügung stehenden Plätzen. Aufgrund der sehr guten Bewerberlage und den kapazitiven Möglichkeiten wurde entschieden, anhand der beschriebenen Auswahlkriterien, zwei Studierende mehr aufzunehmen. Seit dem Wintersemester 2016/2017 konnten somit insgesamt 62 Studierende in das Programm aufgenommen werden. Hinsichtlich der sozio-demografischen Merkmale zeigt sich ein sehr heterogenes Bild innerhalb der bisherigen Teilnehmenden (vgl. Tabelle 1).
Die ersten Evaluationsergebnisse beziehen sich auf die Praxisphasen, die begleitende Seminarreihe sowie den jährlichen Tagesausflug, Stand Ende Sommersemester 2019. Langfristig sollen darüber hinaus in einer Verbleibstudie Effekte auf die Motivation für eine hausärztliche Tätigkeit (auf dem Land) sowie Karriereverläufe abgebildet werden.

Alle Teilnehmenden absolvieren pro Semester bzw. zweimal im Jahr ein Praktikum im Umfang von zwei bis fünf Tagen in ihrer jeweiligen Hausarztpraxis. Jede Hausarztpraxis muss insgesamt mindestens zweimal besucht werden, bevor es zu einem Wechsel kommt. Eine Evaluation findet dann pro Praxis und nicht pro Praktikum statt. In der Abbildung 2 sind die bisherigen Ergebnisse der ersten beiden Jahrgänge abgebildet. Es zeigt sich eine sehr hohe Zufriedenheit bezüglich der praktischen Programmbestandteile. Knapp 95 \% berichten ferner von einem subjektiven Wissenszuwachs und stufen die Praktika als nützlichen Abschnitt ihrer ärztlichen Ausbildung ein. Im Durchschnitt werden die jeweiligen Praktika mit der Schulnote 1,51 bewertet $(\mathrm{N}=37)$.

Pro Semester finden zwei Seminartermine à zwei Zeitstunden statt. Die Dozent*innen und auch deren Anzahl (zwischen einem und drei Dozent*innen pro Seminar) variieren dabei in Abhängigkeit des jeweiligen Themas. Abbildung 2 zeigt, dass die Ansprüche der Studierenden an die Organisation und den inhaltlichen Aufbau der Seminare nahezu vollständig erfüllt wurden. Eine Vermittlung relevanter Inhalte durch die Seminare bestätigten 93\% und einen Wissenszuwachs ebenfalls 93\% der Studierenden. Das Engagement der Dozent*innen und deren strukturierte Darstellung des Lehrstoffs wurden zudem bis auf eine Nennung durchgehend positiv bewertet.

Seit Beginn der „Landpartie 2.0“ haben bisher drei Tagesausflüge zu unterschiedlichen innovativen Gesundheitsmodellen in jeweils einem der beteiligten Landkreise stattgefunden. Ein Ausflugsziel war beispielsweise das Ärztehaus Weilrod im Hochtaunuskreis. In dem medizinischen Versorgungszentrum arbeiten insgesamt sechs Hausärzt*innen als Praxisgemeinschaft in einer Gemeinschafts- und vier Einzelpraxen zusammen. Über einen einst eigens initiierten Qualitätszirkel aus dem auch die Organisation des regionalen Bereitschaftsdienstes hervorging, arbeiten die Ärzt*innen mit weiteren Facharztkolleg*innen im Umkreis eng zusammen. Ausflugsziele wie das Ärztehaus Weilrod bieten den Teilnehmenden somit Einblicke in das vielfältige Spektrum an ärztlichen sowie interprofessionellen Kooperationsmodellen [20]. Die Evaluationsergebnisse zeigen, dass die Erwartungen der Studierenden durchgehend erfüllt wurden (siehe Abbildung 2). Große Zustimmung fand die Organisation. Bei 91\% der Studierenden konnte der Tagesausflug zu einem Wissenszuwachs beitragen und 94\% fühlten sich über neue berufliche Möglichkeiten informiert. 98\% würden den Tagesausflug weiterempfehlen. Im Mittel wurde der Tagesausflug mit der Schulnote 1,43 bewertet $(\mathrm{N}=47)$. 
Tabelle 1: Sozio-demografische Angaben zum Bewerbungszeitpunkt (ca. November eines jeden Jahres); (Quelle: eigene Angaben).

\begin{tabular}{|c|c|c|c|c|c|c|c|c|}
\hline & \multicolumn{2}{|l|}{ Semester } & \multicolumn{2}{|c|}{ Geschlecht } & \multirow[b]{2}{*}{$\begin{array}{l}\text { Altersdurchschnitt } \\
\text { in Jahren } \\
\text { [Altersspanne] }\end{array}$} & \multicolumn{2}{|c|}{ Herkunft (Anzahl) } & \multirow{2}{*}{$\begin{array}{l}\text { Abgeschlossene } \\
\text { Berufsausbildung } \\
\text { (Anzahl) }\end{array}$} \\
\hline & $\begin{array}{l}\text { Erstes klinisches } \\
\text { Semester }\end{array}$ & $\begin{array}{l}\text { Drittes } \\
\text { klinisches } \\
\text { Semester }\end{array}$ & Männlich & Weiblich & & $\begin{array}{l}\text { Ländlich } \\
\text { bis } 10.000 \\
\text { Einwohner }\end{array}$ & $\begin{array}{l}\text { Städtisch } \\
>10.000 \\
\text { Einwohner }\end{array}$ & \\
\hline $\begin{array}{l}\text { Kohorte } \\
2016 / 17\end{array}$ & 11 & 5 & 7 & 9 & $\begin{array}{c}26 \\
{[20-48]}\end{array}$ & 6 & 10 & 4 \\
\hline $\begin{array}{l}\text { Kohorte } \\
2017 / 18\end{array}$ & 12 & 3 & 3 & 12 & $\begin{array}{c}22 \\
{[20-25]}\end{array}$ & 4 & 11 & 1 \\
\hline \multirow{2}{*}{$\begin{array}{l}\text { Kohorte } \\
2018 / 19\end{array}$} & \multirow[t]{2}{*}{13} & \multirow[t]{2}{*}{1} & \multirow{2}{*}{7} & \multirow{2}{*}{7} & \multirow{2}{*}{$\begin{array}{c}27 \\
{[21-37]}\end{array}$} & 8 & 5 & \multirow{2}{*}{9} \\
\hline & & & & & & \multicolumn{2}{|c|}{1 "weiß nicht" } & \\
\hline $\begin{array}{l}\text { Kohorte } \\
2019 / 20\end{array}$ & 17 & 0 & 2 & 15 & $\begin{array}{c}23 \\
{[20-29]}\end{array}$ & 12 & 5 & 3 \\
\hline \multirow{2}{*}{ Gesamt } & 53 & 9 & 19 & 43 & \multirow{2}{*}{$\begin{array}{c}24 \\
{[20-48]}\end{array}$} & \multirow{2}{*}{30} & \multirow{2}{*}{31} & \multirow{2}{*}{17} \\
\hline & \multicolumn{4}{|c|}{62} & & & & \\
\hline
\end{tabular}

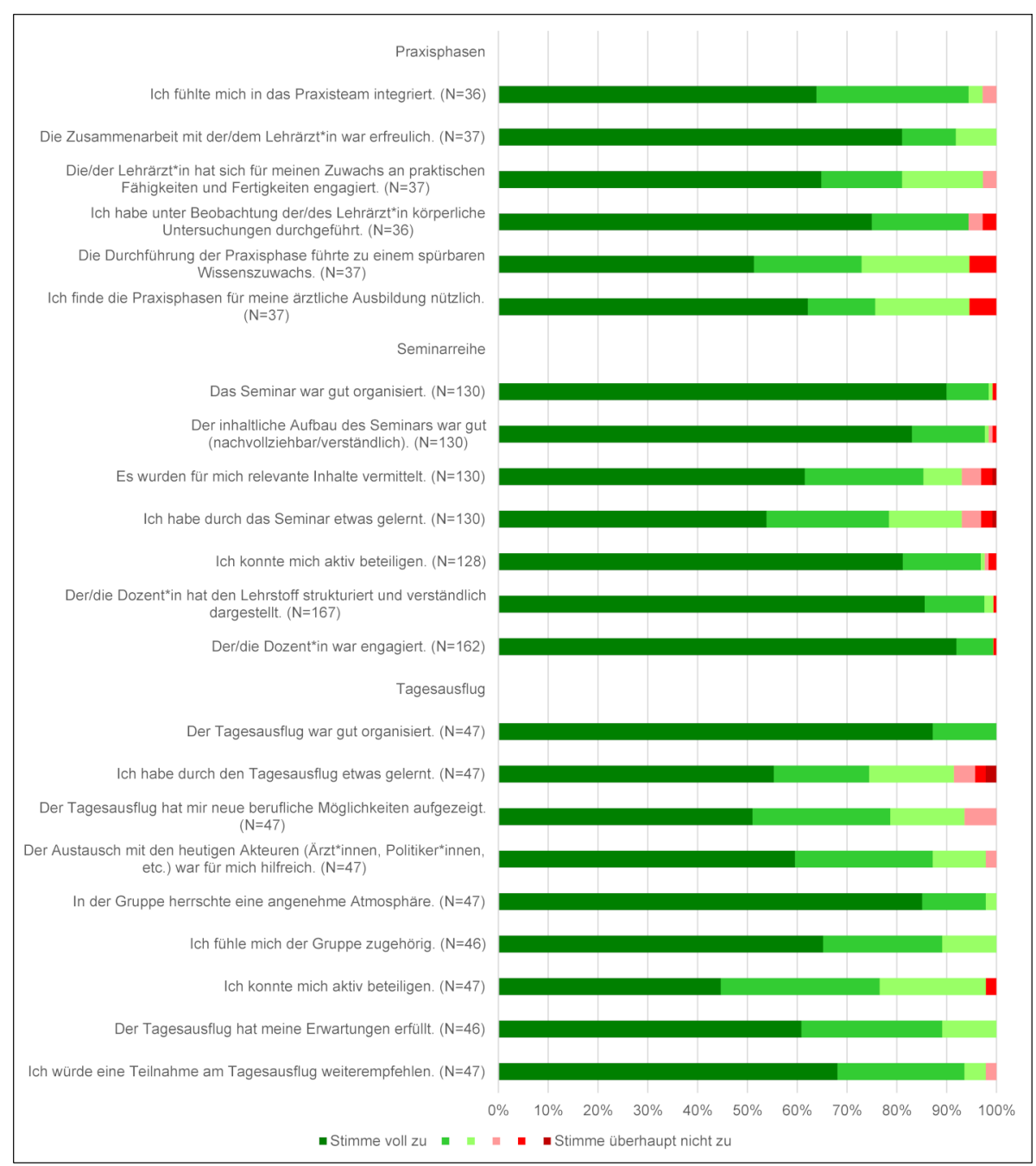

Abbildung 2: Evaluationsergebnisse der Praxisphasen, der Seminarreihe und des Tagesausflug bis Sommersemester 2019 (Quelle: eigene Darstellung)

\section{Diskussion}

Durch einen breit angelegten methodischen Ansatz konnte das Schwerpunktprogramm der „Landpartie 2.0“ von 2015 bis 2016 konzeptionell entwickelt und zum Wintersemester 2016/2017 implementiert werden.
Die „Landpartie 2.0“ beinhaltet im Kern regelmäßige Praxisphasen in ausgewählten und geschulten Landarztpraxen in den hessischen Landkreisen Bergstraße, Fulda und dem Hochtaunuskreis. Umrahmt werden die Praktika von Seminaren. Weitere Bestandteile sind der „Kurs Allgemeinmedizin“ in Kleingruppen sowie ein jährlicher Ta- 
gesausflug zu innovativen Gesundheitsmodellen. Um zeitlichen Mehraufwand zu vermeiden bzw. möglichst gering zu halten, absolvieren die Studierenden automatisch die curricularen Pflichtlehrveranstaltungen „Klinisches Wahlfach“, „Kurs Allgemeinmedizin“ und das „Blockpraktikum Allgemeinmedizin“.

Seit der Einführung des Lehrangebots konnten 62 interessierte Studierende für eine Teilnahme gewonnen werden. Das Verhältnis von Frauen $(69,4 \%)$ zu Männern (30,6\%) ähnelt dabei dem im gesamten Medizinstudium, schwankt jedoch innerhalb der Jahrgänge. Im Durchschnitt sind die Teilnehmenden zum Bewerbungszeitpunkt 24 Jahre alt [Range: 20-48 Jahre] und erwartungsgemäß verfügen ältere Bewerber*innen häufiger über eine abgeschlossene Berufsausbildung. Überraschend ist hingegen, dass ca. die Hälfte aller Studierenden aus Gemeinden mit mehr als 10.000 Einwohnern stammt und somit, der dem Programm zugrundeliegenden Definition, nicht ländlich aufgewachsen ist. Dieses Ergebnis deckt sich jedoch mit den Freitextantworten aus den Bewerbungsbögen. Während einige Bewerber*innen wiederkehrend ihren persönlichen Bezug zum Leben auf dem Land zum Ausdruck bringen, schreiben andere Studierende explizit, dass sie aus einer Großstadt stammen, aber im Rahmen des Programms durchaus die Chance nutzen wollen, andere Lebensstile und womöglich auch eine andere Art der hausärztlichen Medizin kennenlernen zu wollen.

Wie von Fachvertretern und Studierenden gefordert [21], [22], soll durch eine Programmteilnahme der praktische Ausbildungsanteil im Fach Allgemeinmedizin bereits während des Studiums gesteigert werden. Die Teilnehmenden sollen durch einen frühzeitigen und intensiveren Patientenkontakt ihre praktischen, kommunikativen sowie sozialen Kompetenzen weiterentwickeln bzw. festigen. Die Lehrärzt*innen sowohl in den Praxen als auch in den Seminaren dienen den Studierenden als Rollenvorbilder bzw. Mentor*innen und können so das Interesse an der Allgemeinmedizin stärken [23]. Der fakultative Charakter des Angebots ermöglicht den Teilnehmenden sowohl in eine hausärztliche als auch in eine landärztliche Tätigkeit „hineinzuschnuppern“, ohne dabei langfristige Verpflichtungen, wie beispielweise eine Niederlassung in einem der drei beteiligten Landkreise, eingehen zu müssen. Der wiederkehrende und frühzeitige Kontakt zur Allgemeinmedizin und zur Versorgung auf dem Land kann darüber hinaus zu einem „Klebeeffekt“ führen. Evaluationsergebnisse aus vergleichbaren Programmen zeigen einen positiven Effekt auf die Bereitschaft, nach dem Studium hausärztlich (in ländlichen Gebieten) tätig zu werden [11]. Somit stellt das Angebot der „Landpartie 2.0“ eine Alternative zu der bereits in einigen Bundesländern neu eingeführten „Landarztquote“ dar. Während die „Landarztquote“ eine verpflichtende Entscheidung für eine hausärztliche Tätigkeit im ländlichen Raum bereits vor dem Studium fordert [24], bietet das hier beschriebene Schwerpunktprogramm eine auf Attraktivität und intrinsischer Motivation beruhende Möglichkeit, sich selbst als angehende*r Mediziner*in ausprobieren zu dürfen, seine persönlichen Interessen zu prüfen und gleichzeitig die eigenen fachspezifischen Fähigkeiten und Kompetenzen weiterzuentwickeln.

Die bisherigen Auswertungsergebnisse bestätigen, dass die Erwartungen und Anforderungen der Studierenden an die „Landpartie 2.0“ erfüllt werden. So zeigt sich eine hohe bis sehr hohe Zufriedenheit hinsichtlich der Praxisphasen, Seminarreihe und des jährlichen Tagesausflugs. Mittel- bis langfristig angelegte evaluative Verbleibstudien sollen zeigen, ob eine Teilnahme am Angebot das Interesse an der Allgemeinmedizin erhöht und ob die Wahrscheinlichkeit steigt, dass sich Teilnehmende gegenüber Nicht-Teilnehmenden mit ähnlicher Interessenslage eher für ein PJ-Wahltertial und/oder die Weiterbildung Allgemeinmedizin bzw. eine hausärztliche Tätigkeit (in ländlichen Regionen) entscheiden.

Die vorliegenden Evaluationsergebnisse reihen sich in Untersuchungen anderer in Deutschland etablierter Programme ein. Die „Klasse Allgemeinmedizin“ bietet 20 Studierenden pro Jahr seit 2011 ein Wahlpflichtfach fokussierend auf die Primärversorgung an. Eine Evaluation nach den ersten beiden Jahren zeigte, dass der Kompetenzerwerb durch Programmteilnahme einen positiven Einfluss auf die Präferenz zur Fachgebietswahl Allgemeinmedizin hat [9]. Die Untersuchung eines vorklinischen Wahlfachs mit Fokus auf die hausärztliche Versorgung im ländlichen Raum in Leipzig belegte, dass eine Teilnahme am Kurs das Fach Allgemeinmedizin als erste Karriereoption bestärkt [10].

Trotz des umfassenden, methodischen Ansatzes in der Entwicklungsphase kann nicht sicher angenommen werden, dass das entwickelte Angebot der „Landpartie 2.0“ in Zukunft auf das Interesse der Medizinstudierenden stoßen wird. Auch die Frage, inwieweit eine Teilnahme am Programm zu einer höheren Bereitschaft führt, sich z.B. für ein PJ-Wahltertial in der Allgemeinmedizin bzw. darüberhinausgehend für eine hausärztliche Facharztweiterbildung mit anschließender Tätigkeit im ländlichen Raum zu entscheiden, bleibt derzeit noch unklar. Dabei muss vor allem berücksichtigt werden, dass es sich bei den teilnehmenden Studierenden a priori um eine stark selbst-selektierte Gruppe handelt - nur Studierende, die ein grundlegendes Interesse an der Allgemeinmedizin haben und eine entsprechende Facharztweiterbildung sowie die Niederlassung abseits einer Metropole zumindest in Erwägung ziehen, werden sich voraussichtlich für das Lehrangebot bewerben. Von diesem methodischen Problem sind jedoch auch andere vergleichbare Angebote betroffen [7], [25]. Die Berücksichtigung dieses Selektionseffekts ist in weiterführenden Evaluationen folglich unerlässlich.

Insgesamt handelt es sich unseres Wissens nach bei diesem Schwerpunktprogramm um das erste longitudinale Angebot in Deutschland, das auf eine Förderung der Allgemeinmedizin im ländlichen Raum während des Studiums fokussiert. Ähnliche Programme andernorts setzen dagegen vorrangig auf die Allgemeinmedizin ohne explizit ländlichen Fokus. Durch die gewählte methodische Breite in der konzeptionellen Entwicklung des Angebots konnten frühzeitig Best-Practice-Beispiele, die Anforderungen und 
Wünsche der Zielgruppe selbst sowie die geltenden curricularen bzw. rechtlichen Rahmenbedingungen in Deutschland berücksichtigt werden. So wurde in der Entwicklungsphase beispielsweise deutlich, über welche langfristig gesicherten personellen sowie finanziellen Ressourcen das Programm verfügen muss, um eine nachhaltige Umsetzung zu garantieren. Es sollte zudem auf freiwilliger Basis ab dem klinischen Studienabschnitt stattfinden und alle Bestandteile sollten nach Möglichkeit einen Fokus auf praktische Ausbildungsaspekte legen.

\section{Schlussfolgerung}

Das fakultative Programm der „Landpartie 2.0“ konnte erfolgreich konzipiert und in den klinischen Studienabschnitt integriert werden. Das gewählte methodische Vorgehen hat dazu beigetragen, von Beginn an Möglichkeiten und Grenzen eines solchen Angebots zu erkennen und in dessen konzeptioneller Entwicklung zu berücksichtigen.

Erste Evaluationsergebnisse verweisen darauf, dass die „Landpartie 2.0“ den Bedarf der Studierenden erfüllt, einen wertvollen Beitrag in der medizinischen Ausbildung leistet und trotz ihres ländlichen Fokus auch Studierende aus urbaneren Regionen anspricht. Insgesamt kann das entwickelte Schwerpunktprogramm aus Sicht der Autoren aufgrund seines longitudinalen und umfassenden Charakters den weiteren Ausbildungs- und Berufsweg von Medizinstudierenden im Sinne einer hausärztlichen Tätigkeit positiv beeinflussen. Somit stellt die Teilnahme an dem freiwilligen, studienintegrierten Lehrangebot, eine vielversprechende Alternative zu der sogenannten „Landarztquote“ dar.

Eine solche fachspezifische Nachwuchssicherung bedarf jedoch entsprechender personeller beziehungsweise finanzieller Ressourcen, die aktuell an vielen allgemeinmedizinischen Standorten (noch) nicht zur Verfügung stehen. Der wiederholt beteuerten Bereitschaft zur Förderung der Allgemeinmedizin im Studium müssen daher bundesweit konkrete Schritte, vor allem nachhaltig finanzierte Ressourcen zur Umsetzung entsprechender Maßnahmen, folgen.

\section{Anmerkungen}

${ }^{1}$ Die Landpartie 1.0. ermöglicht interessierten Studierenden in Kooperation mit dem Landkreis Fulda die Durchführung des Blockpraktikums Allgemeinmedizin in landärztlichen Praxen und wird durch besondere Angebote (Eventtag, Freizeitgutschein, Fahrt- und Übernachtungskostenübernahme) ergänzt.

${ }^{2}$ Die Praxen sollen in Gemeinden mit weniger als 20.000 Einwohnern liegen. Bis auf zwei Ausnahmen (jeweils ca. 25.000 Einwohner*innen) wird dieses Kriterium durchgehend erfüllt.

\section{Danksagung}

Unser Dank geht an alle Praxen, Landkreise, Fachvertreter sowie Studierende, die uns bei der Entwicklung der „Landpartie 2.0“ tatkräftig unterstützt haben.

\section{Förderung}

Diese Arbeit wurde durch die Kreisausschüsse der Landkreise Bergstraße, Fulda und dem Hochtaunuskreis unterstützt.

\section{Interessenkonflikt}

Die Autor*innen erklären, dass sie keinen Interessenkonflikt im Zusammenhang mit diesem Artikel haben.

\section{Literatur}

1. Deutsch T, Lippmann S, Sandholzer H. Gewinnung hausärztlichen Nachwuchses - Zusammenhang zwischen praxisorientierter Lehre und Karriereentscheidung. Gesundheitswesen. 2014;76(01):2631. DOI: $10.1055 / \mathrm{s}-0033-1334933$

2. Nicholson S, Hastings AM, McKinley RK. Influences on students' career decisions concerning general practice: a focus group study. Br J Gen Pract..2016;66(651):e768-775. DOI: 10.3399/bjgp16X687049

3. Krupa LK, Chan BT. Canadian rural family medicine training programs: growth and variation in recruitment. Can Fam Physician. 2005;51:852-853.

4. Eley DS, Synnott R, Baker PG, Chater AB. A decade of Australian rural clinical school graduates - where are they and why? Rural Remote Health. 2012;12:1937. Zugänglich unter/available from: http://www.rrh.org.au/publishedarticles/article_print_1937.pdf

5. Rabinowitz HK, Diamond JJ, Markham FW, Wortman JR. Medical school programs to increase the rural physician supply: a systematic review and projected impact of widespread replication. Acad Med. 2008;83(3):235-243. DOI: 10.1097/ACM.0b013e318163789b

6. Barthen L, Beig I, Sennekamp M, Gerlach FM, Erler A, RavensTaueber G. Raus aufs Land während des Medizinstudiums: Eine Übersicht zu bestehenden und geplanten allgemeinmedizinischen Förderangeboten. Z Allg Med. 2016;92(11):448-445. DOI: 10.3238/zfa.2016.0448-0455

7. Blozik E, Ehrhardt M, Scherer M. Förderung des allgemeinmedizinischen Nachwuchses: Initiativen in der universitären Ausbildung von Medizinstudierenden. Bundesgesundhbl Gesundheitsforsch Gesundheitsschutz. 2014;57(7):892-902. DOI: 10.1007/s00103-014-1984-6

8. Schäfer H, Mangold B, Paulitsch M, Gerlach FM. Landpartie Fulda - ein Projekt zur Förderung des hausärztlichen Nachwuchses im ländlichen Raum. Z Allg Med. 2015;91(6):260-263. DOI: 10.3238/zfa.2015.0260-0263

9. Samos FA, Heise M, Fuchs S, Mittmann S, Bauer A, Klement A. A pilot phase evaluation of the elective general pracitice class: results of student surveys of the first two years. GMS J Med Educ. 2017;34(1):Doc4. DOI: 10.3205/zma001081 
10. Deutsch T, Hönigschmid P, Frese T, Sandholzer H. Early community-based family practice elective positively influences medical students' career considerations - a pre-post-comparison BMC Fam Pract. 2013;14:24. DOI: 10.1186/1471-2296-14-24

11. Goodfellow A, Ulloa JG, Dowling PT, Talamantes E, Chheda S, Bone C, Moreno G. Predictors of primary care physician practice location in underserved urban or rural areas in the United States: A systematic literature review. Acad Med. 2016;91(9):13131321. DOI: $10.1097 /$ ACM.0000000000001203

12. Bayerisches Ärzteblatt. Hausarztleben im Bayerischen Wald. Bay Ärztebl. 2016. Zugänglich unter/available from: https:// www.bayerisches-aerzteblatt.de/inhalte/details/news/detail/ News/hausarztleben-im-bayerischen-wald.html

13. Nohl-Deryk P. Landarzt-Famulaturen: Heiss begehrt. Ärztebl. 2015. Zugänglich unter/available from: https:// www.aerzteblatt.de/archiv/172217/Landarzt-Famulaturen-Heissbegehrt

14. Barthen L, Ravens-Taeuber G, Paulitsch MA, Gerlach FM, Sennekamp M. How can General Practice be incorporated Iongitudinally in medical studies? Students' views on the development of a new rural health program. GMS J Med Educ. 2018;35(3):Doc42. DOI: 10.3205/zma001188

15. Yengo-Kahn AM, Baker CE, Lomis KD. Medical students' perspectives on implementing curriculum change at one institution. Acad Med. 2017;92(4):455-461. DOI: 10.1097/ACM.0000000000001569

16. Deutsch T, Lippmann S, Frese T, Sandholzer H. Who wants to become a general practitioner? Student and curriculum factors associated with choosing a GP career - a multivariable analysis with particular consideration of practice-orientated GP courses. Scand J Prim Health Care. 2015:33(1):47-53. DOI: 10.3109/02813432.2015.1020661

17. Puddey I, Mercer A, Playford, D, Pougnault S, Riley G. Medical student selection criteria as predictors of intended rural practice following graduation. BMC Med Eeduc. 2014;14(1):218. DOI: 10.1186/1472-6920-14-218

18. Viscomi M, Larkins S, Gupta TS. Recruitment and retention of general practitioners in rural Canada and Australia: a review of the literature. Can J Rural Med. 2013;18(1):13-23.

19. Ravens-Taeuber G, Wunder A, Güthlin C, Koné I. Checkliste Blockpraktikum Allgemeinmedizin. Wie beeinflusst die systematische Festlegung von ausgewählten Lernzielen den subjektiven Lernfortschritt der Studierenden? Z Allg Med. 2019;95(7):448-445. DOI: 0.3238/zfa.2019.0307-0313

20. Ärztehaus Weilrod. Innovative Gesundheitsmodelle. Weilrod: Ärztehaus Weilrod. Zugänglich unter/available from:http:// www.innovative-gesundheitsmodelle.de/modelle/-/asset_ publisher/ubf7syrf6E2N/content/arztehaus-weilrod/maximized

21. Bundesvertretung der Medizinstudierenden in Deutschland (bvmd). Longitudinale Einbindung der Allgemeinmedizin in das Medizinstudium. Berlin: bvmd; 2014. Zugänglich unter/available from: https://www.bvmd.de/fileadmin/redaktion/ Positionspapiere/Positionspapier_2014-04-26_Longit. Einbindung-der-Allgemeinmed-in-Medizinstudium.pdf
22. Huenges B, Gulich M, Böhme K, Fehr F, Streitlein-Böhme I, Rüttermann V, Baum E, Niebling WB, Rusche $\mathrm{H}$. Recommendations for undergraduate training in the primary care sector - position paper of the GMA-Primary Care Committee. GMS Z Med Ausbild. 2014;31(3):Doc35. DOI: 10.3205/zma000927

23. Stagg P, Prideaux D, Greenhill J, Sweet L. Are medical students influenced by preceptors in making career choices, and if so how? A systematic review. Rural Remote Health. 2012;12:1832.

24. Bundesministerium für Bildung und Forschung. Masterplan Medizinstudium 2020. Berlin: Bundesministerium für Bildung und Forschung; 2017. Zugänglich unter/available from: https:/ /www.bmbf.de/files/2017-03-31_Masterplan\% 20Beschlusstext.pdf

25. Verma P, Ford JA, Stuart A, Howe A, Everington S, Steel N. A systematic review of strategies to recruit and retain primary care doctors. BMC Health Serv Res. 2016;16:126. DOI: 10.1186/s12913-016-1370-1

\section{Korrespondenzadresse:}

Linda Seeger, M.Sc. Public Health

Goethe-Universität Frankfurt am Main, Institut für

Allgemeinmedizin, Theodor-Stern-Kai 7, 60590

Frankfurt/Main, Deutschland

seeger@allgemeinmedizin.uni-frankfurt.de

\section{Bitte zitieren als}

Seeger L, Becker N, Ravens-Taeuber G, Sennekamp M, Gerlach FM.

"Landpartie 2.0" - Conceptual development and implementation of a longitudinal priority program to promote family medicine in rural areas. GMS J Med Educ. 2020;37(3):Doc29.

DOI: 10.3205/zma001322, URN: urn:nbn:de:0183-zma0013225

\section{Artikel online frei zugänglich unter}

https://www.egms.de/en/journals/zma/2020-37/zma001322.shtml

Eingereicht: 22.10 .2019

Überarbeitet: 15.01 .2020

Angenommen: 11.02.2020

Veröffentlicht: 15.04.2020

\section{Copyright}

(C2020 Seeger et al. Dieser Artikel ist ein Open-Access-Artikel und steht unter den Lizenzbedingungen der Creative Commons Attribution 4.0 License (Namensnennung). Lizenz-Angaben siehe

http://creativecommons.org/licenses/by/4.0/. 


\section{Darstellung des eigenen Anteils an den einzelnen Publikationen}

Die vorliegende Dissertation wurde am Institut für Allgemeinmedizin der GoetheUniversität Frankfurt unter der Betreuung von Prof. Dr. med. Ferdinand M. Gerlach und Dr. phil. Monika Sennekamp durchgeführt. Die einzelnen Arbeitsschritte und der jeweilige Arbeitsanteil der Autorin an den einzelnen Publikationen ist im Folgenden dargestellt:

\section{Arbeitsschritte}

Arbeitsanteil

\section{Erste Publikation}

Literaturrecherche

Vollständig

Kontaktaufnahme und Austausch mit Experten

Vollständig

Befragung aller medizinischen Fakultäten

Vorwiegend

Entwicklung des Leitfadens

Vorwiegend

Datenerhebung - Kontaktaufnahme per E-Mail, Telefongespräche mit

Vorwiegend Fachvertretern

Datenauswertung mittels Excel

Vorwiegend

Verfassen der Publikation

Vollständig

Korrektur der Publikation im Zuge des Review-Prozesses

Vollständig

\section{Zweite Publikation}

Fragebogenentwicklung

Vorwiegend

Korrespondenz mit Dekanat

Vollständig

Datenerhebung mittels Survey Monkey

Vollständig

Datenauswertung mittels SPSS und Excel

Vorwiegend

Verfassen der Publikation

Vollständig

Korrektur der Publikation im Zuge des Review-Prozesses

Vollständig

\section{Dritte Publikation}

Konzeptionelle Entwicklung der Landpartie 2.0 auf Basis der vorangegangenen Ergebnisse

Vorwiegend

Entwicklung und Durchführung der Evaluation

Vorwiegend

Auswertung der Evaluationsdaten mittels SPSS und Excel

Vorwiegend

Verfassen der Publikation

Vollständig

Korrektur der Publikation im Zuge des Review-Prozesses

Vollständig 


\section{Anhang}

Fragebogen

Entwicklung eines fakultativen Landarztprogramms/Schwerpunktprogramms

\section{ALLGEMEINE ANGABEN \\ 1) Geburtsjahr

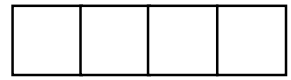 \\ 2) Fachsemester Medizinstudium

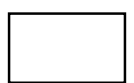 \\ 3) Geschlecht \\ weiblich \\ männlich}

4) Wie viele Einwohner hat der Ort, in dem Sie hauptsächlich aufgewachsen sind?
$\square<5.000$ Einwohner
$\square>5.000-10.0000$ Einwohner
$\square>10.000-20.000$ Einwohner
$\square>20.000-50.000$ Einwohner
$\square>50.000-100.000$ Einwohner
$>100.000$ Einwohner

5) Haben Sie bereits eine abgeschlossene Berufsausbildung?

Ja, und zwar:

Nein

6) Haben Sie Kinder?

$\square \mathrm{Ja}$

$\square \quad$ Nein

\section{BERUFSWUNSCH UND STUDIUM}

In diesem Abschnitt möchten wir gern etwas über Ihre Berufswünsche und die Bewertung Ihres aktuellen Studiums wissen.

Inwieweit treffen die beiden folgenden Aussagen auf Sie zu?

7) „2 $\mathrm{Zu}$ Beginn meines Studiums konnte ich mir vorstellen, später als niedergelassene/r Hausärztin/Hausarzt zu arbeiten. “

Trifft zu

Trifft nicht zu

8) „Ich kann mir jetzt vorstellen, später als niedergelassene/r Hausärztin/Hausarzt zu arbeiten.““

Trifft zu

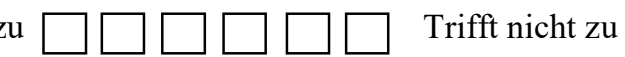

9) Wenn Sie Ihren späteren Arbeitsort völlig frei wählen könnten: Wie viele Einwohner sollte der Ort mindestens haben?
$>100.000$ Einwohner
$>50.000-100.000$ Einwohner
$>20.000-50.000$ Einwohner
$>10.000-20.000$ Einwohner
$>5.000-10.0000$ Einwohner
$<5.000$ Einwohner
Ich habe noch keine Priorität 
Weiß nicht

10) Zu welchem Zeitpunkt sollten Medizinstudierende Ihrer Meinung nach erstmalig praktische Erfahrungen in einer hausärztlichen Praxis sammeln?

$\square$ Bereits vor dem Studium (Vorpraktikum)

Im 8. Semester (Klinik)

Im 1. Semester (Vorklinik)

$\square$ Im 9.Semester (Klinik)

$\square \quad$ Im 2. Semester (Vorklinik)

Im 10. Semester (Klinik)

$\square \quad$ Im 3. Semester (Vorklinik)

$\square$ Im 11. Semester (Praktisches Jahr)

$\square$ Im 4. Semester (Vorklinik)

Im 12. Semester (Praktisches Jahr)

$\square$ Im 5. Semester (Klinik)

Weiß nicht

$\square \quad$ Im 6. Semester (Klinik)

Sonstiger Beginn:

$\square \quad \operatorname{Im}$ 7. Semester (Klinik)

11) Angenommen für Sie kommt eine hausärztliche Tätigkeit in einer ländlichen Region in Frage: Fühlen Sie sich dazu durch Ihr bisheriges Studium ausreichend vorbereitet?

$\mathrm{Ja}$

Nein

Zum Teil

$\mathrm{Zu}$ früher Zeitpunkt im Studium, um dies beurteilen zu können

Weiß nicht

12) Wenn NEIN oder ZUM TEIL angekreuzt wurde:

Was bräuchten Sie, damit Sie sich ausreichend auf eine Tätigkeit als Hausärztin/Hausarzt auf dem Land vorbereitet fühlen?

\section{ANGABEN ZUM ANGEBOT}

\section{BITTE LESEN SIE SICH DEN FOLGENDEN ABSCHNITT AUFMERKSAM DURCH.}

Das noch zu entwickelnde, freiwillige Angebot soll sich aus folgenden Teilen zusammensetzen:

1. Praktischer Teil: Mitarbeit/Praktikum in Hausarztpraxen in einer ländlichen Region

2. Kleingruppenseminare: $\underline{\text { z.B. }}$. Vor- und Nachbereitung des praktischen Teils, Expertengespräche, Referate

3. Mentoringprogramm: langfristige Betreuung durch Hausärztin/Hausarzt 
Ziel ist es, Ihnen ein realistisches Bild von einer hausärztlichen Tätigkeit auf dem Land zu vermitteln.

Damit Sie nur einen sehr geringen oder möglichst keinen Mehraufwand haben, wollen wir das Angebot als klinisches Wahlfach konzipieren und somit in Ihr reguläres Studium einbauen.

Denkbar ist zudem, dass weitere Pflichtlehrveranstaltungen, wie z.B. das vorklinische Wahlfach, die Famulatur, der Kurs 1 Allgemeinmedizin, das Blockpraktikum Allgemeinmedizin und/oder ein Tertial im Praktischen Jahr in das freiwillige Angebot einbezogen werden.

13) Angenommen Sie wären entschlossen, das Angebot zu belegen: Haben Sie Ideen/Anregungen/Wünsche...

a) ...wie der PRAKTISCHE TEIL (Mitarbeit/Praktikum in Hausarztpraxen in einer ländlichen Region) gestaltet sein sollte, damit er eine sinnvolle Ergänzung zu Ihrem bisherigen Studium darstellt?

(z.B. bezogen auf Inhalt, Organisation, etc.)

b) ...wie die KLEINGRUPPENSEMINARE gestaltet sein sollten, damit diese eine sinnvolle Ergänzung zu Ihrem bisherigen Studium darstellen? (z.B. bezogen auf Themen, Inhalt, Organisation, etc.)

14) Wären Sie an einem begleitenden Mentoringprogramm (Betreuung und Begleitung der Teilnehmerin/des Teilnehmers durch erfahrene Hausärztinnen/Hausärzte) im Rahmen des Angebots interessiert?

$\square \mathrm{Ja}$

$\square \quad$ Nein

Weiß nicht 
15) Welche Erwartungen hätten Sie an ein solches Mentoringprogramm?

16) Wie lang sollte das Angebot, bestehend aus einem praktischen Teil, Kleingruppenseminaren und einem Mentoringprogramm dauern?

$\square$ Ein Semester

$\square$ Mehrere Semester

$\square$ Das gesamte Studium umfassend (Vorklinik, Klinik und ggf. PJ)

$\square$ Egal, solange das Angebot für mich sinnvoll ist

$\square$ Weiß nicht

$\square$ Sonstige Dauer:

17) Würden Sie an einem solchen freiwilligen Angebot, bestehend aus einem praktischen Teil, Kleingruppenseminaren und einem Mentoringprogramm teilnehmen?

$\square \mathrm{Ja}$

$\square$ Nein

$\square$ Weiß nicht

18) Wenn NEIN, warum nicht?

Mehrfachantworten möglich.

$\square$ Das Angebot ist mir bisher zu unkonkret, ich kann mir nichts darunter vorstellen

$\square$ Kein Interesse an der Allgemeinmedizin

$\square$ Kein Interesse an einer Niederlassung

Kein Interesse, in einer ländlichen Region tätig zu werden

Kein Interesse an weiteren Praktika

Das Studium vermittelt mir bereits jetzt alle notwendigen Inhalte

Keine Zeit im Studium

Sonstiges:

19) Haben Sie noch weitere Anregungen/Ideen:

\section{Vielen Dank für Ihre Teilnahme!}




\section{Schriftliche Erklärung}

Ich erkläre ehrenwörtlich, dass ich die dem Fachbereich Medizin der Johann Wolfgang Goethe-Universität Frankfurt am Main zur Promotionsprüfung eingereichte Dissertation mit dem Titel

Landpartie 2.0 - Konzeptionelle Entwicklung und Implementierung eines longitudinalen Schwerpunktprogramms zur Förderung der Allgemeinmedizin im ländlichen Raum

in dem Institut für Allgemeinmedizin unter Betreuung und Anleitung von Prof. Dr. Ferdinand M. Gerlach, MPH mit Unterstützung durch Dr. phil. Monika Sennekamp ohne sonstige Hilfe selbst durchgeführt und bei der Abfassung der Arbeit keine anderen als die in der Dissertation angeführten Hilfsmittel benutzt habe. Darüber hinaus versichere ich, nicht die Hilfe einer kommerziellen Promotionsvermittlung in Anspruch genommen zu haben.

Ich habe bisher an keiner in- oder ausländischen Universität ein Gesuch um Zulassung zur Promotion eingereicht. Die vorliegende Arbeit wurde bisher nicht als Dissertation eingereicht.

Vorliegende Ergebnisse der Arbeit wurden in folgendem Publikationen veröffentlicht:

Barthen L, Beig I, Sennekamp M, Gerlach FM, Erler A, Ravens-Taueber G. Raus aufs Land während des Medizinstudiums: Eine Übersicht $\mathrm{zu}$ bestehenden und geplanten allgemeinmedizinischen Förderangeboten. Z Allg Med. 2016;92(11):448-445. DOI: 10.3238/zfa.2016.0448-0455

Barthen L, Ravens-Taeuber G, Paulitsch MA, Gerlach FM, Sennekamp M. How can General Practice be incorporated longitudinally in medical studies? Students' views on the development of a new rural health program. GMS J Med Educ. 2018;35(3):Doc42. DOI: $10.3205 / \mathrm{zma} 001188$

Seeger L, Becker N, Ravens-Taeuber G, Sennekamp M, Gerlach FM. "Landpartie 2.0" Conceptual development and implementation of a longitudinal priority program to promote family medicine in rural areas. GMS J Med Educ. 2020;37(3):Doc29. DOI: $10.3205 / \mathrm{zma} 001322$

Frankfurt, den 18.3.2021

Ort, Datum

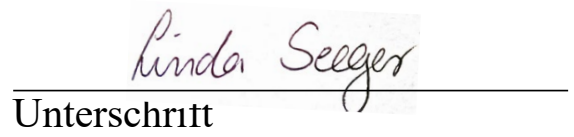

